Old Dominion University

ODU Digital Commons

Summer 2012

\title{
FRIEND: A Cyber-Physical System for Traffic Flow Related Information Aggregation and Dissemination
}

Samy S. El-Tawab

Old Dominion University

Follow this and additional works at: https://digitalcommons.odu.edu/computerscience_etds

Part of the Computer Sciences Commons

\section{Recommended Citation}

El-Tawab, Samy S.. "FRIEND: A Cyber-Physical System for Traffic Flow Related Information Aggregation and Dissemination" (2012). Doctor of Philosophy (PhD), Dissertation, Computer Science, Old Dominion University, DOI: 10.25777/n2gv-mj39

https://digitalcommons.odu.edu/computerscience_etds/104

This Dissertation is brought to you for free and open access by the Computer Science at ODU Digital Commons. It has been accepted for inclusion in Computer Science Theses \& Dissertations by an authorized administrator of ODU Digital Commons. For more information, please contact digitalcommons@odu.edu. 


\section{FRIEND: A CYBER-PHYSICAL SYSTEM FOR TRAFFIC FLOW RELATED INFORMATION AGGREGATION AND DISSEMINATION}

by

Samy S. El-Tawab

B.S. June 2002, Alexandria University, Egypt

M.S. June 2006, Alexandria University, Egypt

A Dissertation Submitted to the Faculty of Old Dominion University in Partial Fulfillment of the Requirements for the Degree of

DOCTOR OF PHILOSOPHY

COMPUTER SCIENCE

OLD DOMINION UNIVERSITY August 2012

Approved by:

St /phan Olariu (Director)

Hussein Abdel-Wahab (Member)

Michele C. Weigle (Member)

TamerNadeem (Member)

Dimitrif C. Popescu (Member) 


\title{
ABSTRACT \\ FRIEND: A CYBER-PHYSICAL SYSTEM FOR TRAFFIC FLOW RELATED INFORMATION AGGREGATION AND DISSEMINATION
}

\author{
Samy S. El-Tawab \\ Old Dominion University, 2012 \\ Director: Dr. Stephan Olariu
}

The major contribution of this thesis is to lay the theoretical foundations of FRIEND A cyber-physical system for traffic Flow-Related Information aggrEgatioN and Dissemination. By integrating resources and capabilities at the nexus between the cyber and physical worlds, FRIEND will contribute to aggregating traffic flow data collected by the huge fleet of vehicles on our roads into a comprehensive, near real-time synopsis of traffic flow conditions. We anticipate providing drivers with a meaningful, color-coded, at-a-glance view of flow conditions ahead, alerting them to congested traffic.

FRIEND can be used to provide accurate information about traffic flow and can be used to propagate this information. The workhorse of FRIEND is the ubiquitous lane delimiters (a.k.a. cat's eyes) on our roadways that, at the moment, are used simply as dumb reflectors. Our main vision is that by endowing cat's eyes with a modest power source, detection and communication capabilities they will play an important role in collecting, aggregating and disseminating traffic flow conditions to the driving public. We envision the cat's eyes system to be supplemented by road-side units (RSU) deployed at regular intervals (e.g. every kilometer or so). The RSUs placed on opposite sides of the roadway constitute a logical unit and are connected by optical fiber under the median. Unlike inductive loop detectors, adjacent RSUs along the roadway are not connected with each other, thus avoiding the huge cost of optical fiber. Each RSU contains a GPS device (for time synchronization), an active Radio Frequency Identification (RFID) tag for communication with passing cars, a radio transceiver for RSU to RSU communication and a laptop-class computing device. The physical components of FRIEND collect traffic flow-related data from passing vehicles. The collected data is used by FRIEND's inference engine to build beliefs about the state of the traffic, to detect traffic trends, and to disseminate relevant traffic flow-related information along the roadway. The second contribution of this thesis is the development of an incident classification and detection algorithm that can be used to classify different types of traffic incident. Then, it can notify the necessary target of the incident. We also compare our incident detection technique with other VANET techniques. 
Our third contribution is a novel strategy for information dissemination on highways. First, we aim to prevent secondary accidents. Second, we notify drivers far away from the accident of an expected delay that gives them the option to continue or exit before reaching the incident location. A new mechanism tracks the source of the incident while notifying drivers away from the accident. The more time the incident stays, the further the information needs to be propagated. Furthermore, the denser the traffic, the faster it will backup. In high density highways, an incident may form a backup of vehicles faster than low density highways. In order to satisfy this point, we need to propagate information as a function of density and time. 
Copyright, 2012, by Samy S. El-Tawab, All Rights Reserved. 


\section{ACKNOWLEDGEMENTS}

I would like to express my gratitude to Allah (God) for providing me the blessings to complete this work. Also, I could not have completed this thesis without the help of many individuals to whom I would like to express my appreciation. First and foremost, I would like to thank my advisor, Professor Stephan Olariu, who has put a great deal of time and effort into the guidance of this work. Professor Olariu supported me scientifically and guided me as his son. His office was always open to help me solve any technical or personal problem I had during the program. Also, I would like to convey my sincere thanks to Professor Hussein Abdel-wahab for his support and help.

Next, I would like to thank my PhD committee Drs. Michele Weigle, Tamer Nadeem and Dimitrie Popescu. Their expertise, thorough reviewing, continuous support, and valuable suggestions have led to a greatly improved dissertation.

I am also grateful to my family for their encouragement and support. Finally, special thanks to my father and mother for their understanding and patience during the time I spend completing this work. 


\section{TABLE OF CONTENTS}

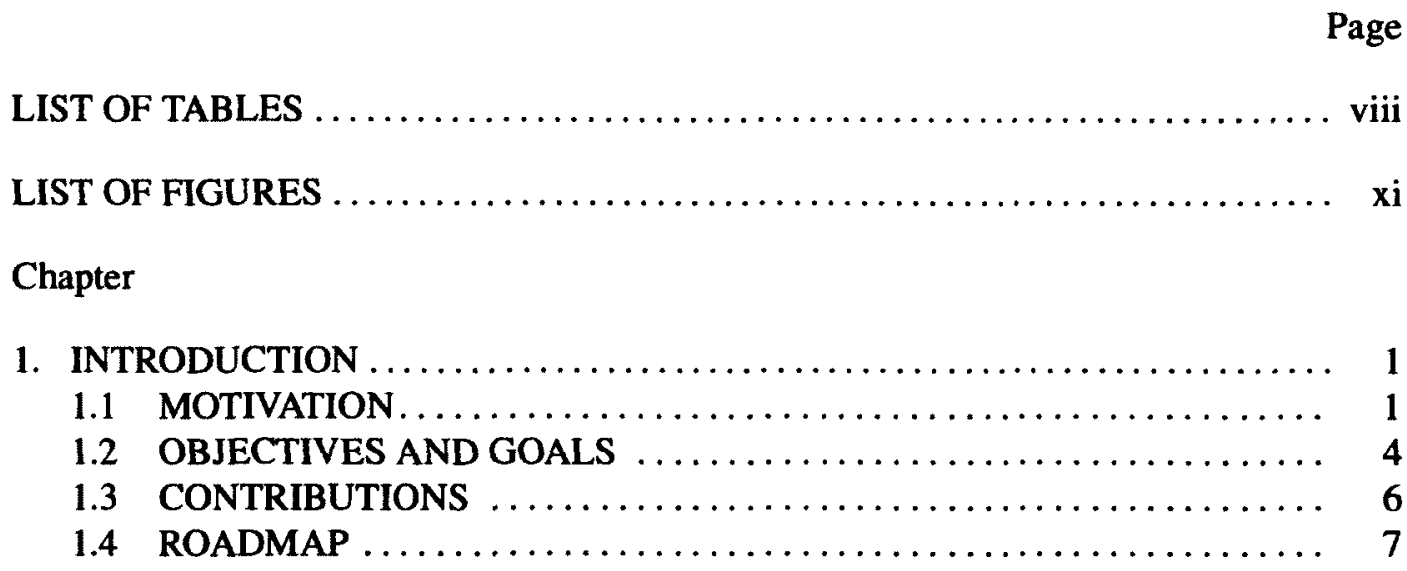

2. TECHNICAL BACKGROUND - REVIEW OF RELEVANT LITERATURE .... 9

2.1 VANET OVERVIEW $\ldots \ldots \ldots \ldots \ldots \ldots \ldots \ldots \ldots \ldots \ldots \ldots \ldots \ldots$

2.2 VEHICLE DETECTION TECHNOLOGY $\ldots \ldots \ldots \ldots \ldots \ldots \ldots \ldots, 12$

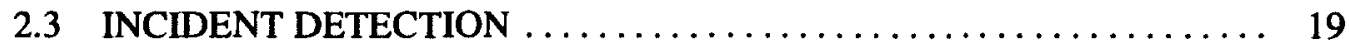

2.4 TRAFFIC MONITORING IN VANET $\ldots \ldots \ldots \ldots \ldots \ldots \ldots \ldots \ldots \ldots, 22$

2.5 INFORMATION DISSEMINATION SYSTEMS $\ldots \ldots \ldots \ldots \ldots \ldots \ldots 24$

2.6 NOTICE: AN ARCHITECTURE FOR THE NOTIFICATION OF TRAF-

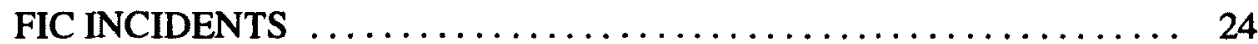

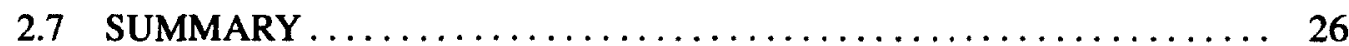

3. FRIEND - PHYSICAL COMPONENTS AND COMMUNICATION PRIMITIVES.

3.1 FRIEND - THE PHYSICAL COMPONENTS $\ldots \ldots \ldots \ldots \ldots \ldots \ldots \ldots . \ldots \ldots$

3.2 DATA COMMUNICATION IN FRIEND $\ldots \ldots \ldots \ldots \ldots \ldots \ldots \ldots \ldots . \ldots \ldots$

3.3 A TAXONOMY OF APPLICATIONS OF FRIEND $\ldots \ldots \ldots \ldots \ldots \ldots .44$

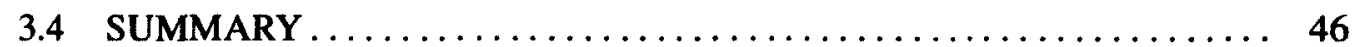

4. REASONING ABOUT TRAFFIC FLOW PARAMETERS $\ldots \ldots \ldots \ldots \ldots \ldots .48$

4.1 TRAFFIC FLOW PARAMETERS $\ldots \ldots \ldots \ldots \ldots \ldots \ldots \ldots \ldots \ldots, 48$

4.2 MORE ON THE HEADWAY DISTANCE $\ldots \ldots \ldots \ldots \ldots \ldots \ldots \ldots, 55$

4.3 REASONING ABOUT THE EXPECTED HEADWAY DISTANCE IN

FREE-FLOW TRAFFIC $\ldots \ldots \ldots \ldots \ldots \ldots \ldots \ldots \ldots \ldots \ldots \ldots, 61$

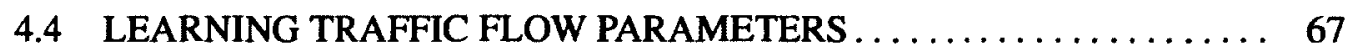

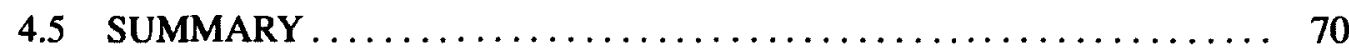

5. COMMUNICATION PROTOCOLS IN FRIEND $\ldots \ldots \ldots \ldots \ldots \ldots \ldots \ldots \ldots \ldots$

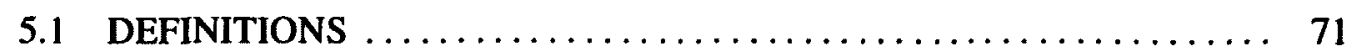

5.2 RSU COMMUNICATION $\ldots \ldots \ldots \ldots \ldots \ldots \ldots \ldots \ldots \ldots \ldots \ldots \ldots, 71$

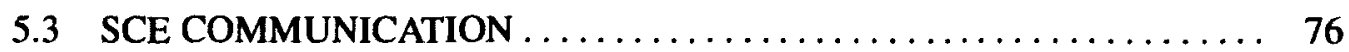




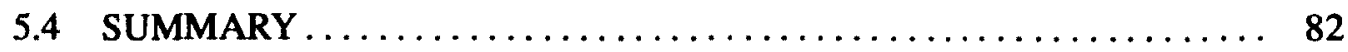

6. MAKING TRAFFIC-RELATED DECISIONS IN FRIEND .............. 84

6.1 REASONING ABOUT THE SAMPLE MEAN OF HEADWAY DISTANCE 85

6.2 COLOR-CODED TRAFFIC STATE $\ldots \ldots \ldots \ldots \ldots \ldots \ldots \ldots \ldots . \ldots . \ldots . \ldots$

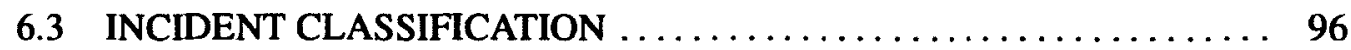

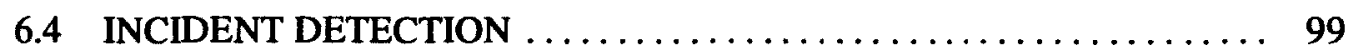

6.5 INCIDENT INFORMATION DISSEMINATION $\ldots \ldots \ldots \ldots \ldots \ldots \ldots 101$

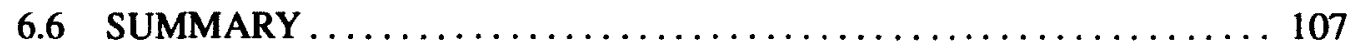

7. SIMULATION, EVALUATION AND APPLICATIONS OF FRIEND $\ldots \ldots \ldots \ldots 108$

7.1 SIMULATION SETUP AND PARAMETERS $\ldots \ldots \ldots \ldots \ldots \ldots \ldots \ldots 108$

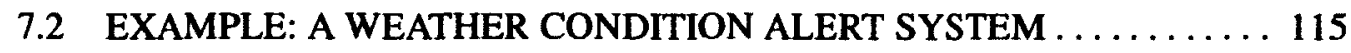

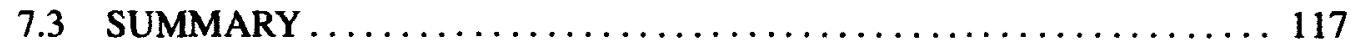

8. CONCLUDING REMARKS AND DIRECTIONS FOR FUTURE WORK . . . 119

8.1 CONCLUDING REMARKS $\ldots \ldots \ldots \ldots \ldots \ldots \ldots \ldots \ldots \ldots \ldots \ldots \ldots$

8.2 DIRECTIONS FOR FUTURE WORK $\ldots \ldots \ldots \ldots \ldots \ldots \ldots \ldots \ldots \ldots$

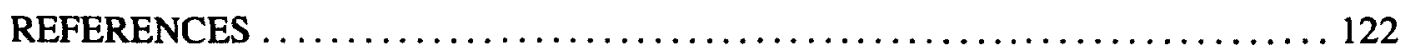

APPENDICES

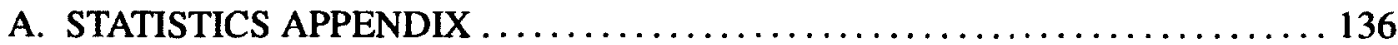

A.1 THE LOG-NORMAL DISTRIBUTION $\ldots \ldots \ldots \ldots \ldots \ldots \ldots \ldots \ldots \ldots$

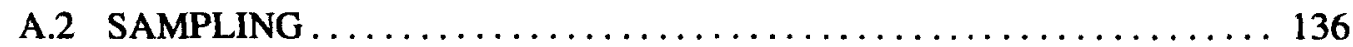

A.3 EULER'S GAMMA FUNCTION - A QUICK REFRESHER . . . . . . . . 137

A.4 EULER'S BETA FUNCTION - A QUICK REFRESHER ............ 137

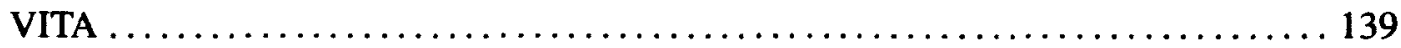




\section{LIST OF TABLES}

Table

Page

1 A sample of detection/sensor technologies.

2 Performance comparison of several incident detection algorithms based on

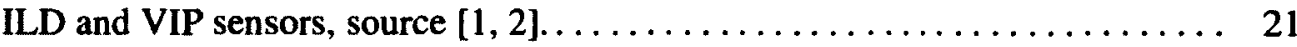

3 Examples of some information dissemination algorithms. .......... 25

4 Different types of communications in FRIEND. . . . . . . . . . . . . . 39

$5 \quad$ Illustrating the difference between active and passive tags. ........... 39

6 A comparison between short-range wireless technologies.............. 40

$7 \quad$ Example of vehicular networking applications. $\ldots \ldots \ldots \ldots \ldots \ldots \ldots \ldots$

8 Our simulation parameters. $\ldots \ldots \ldots \ldots \ldots \ldots \ldots \ldots \ldots \ldots \ldots \ldots \ldots \ldots$

9 Total number of messages sent with percentage of collision. . . . . . . . . 112 


\section{LIST OF FIGURES}

Figure

Page

$1 \quad$ Illustrating the main sources of congestion. .................... 1

2 Illustrating the main infrastructure components of the FRIEND......... 5

3 Example of audio/video highway monitoring system $\ldots \ldots \ldots \ldots \ldots \ldots \ldots$

4 Comparison of detection rates of several incident detection algorithms....... 22

5 Comparison of false alarm rates of several incident detection algorithms. . . . 23

6 Example of a SCE with solar panel: poly-crystalline silicon rechargeable NiMH batteries; http://www.alibaba.com [3] . ................. 29

$7 \quad$ A highway segment with several RSUs. $\ldots \ldots \ldots \ldots \ldots \ldots \ldots \ldots \ldots \ldots$

8 Roadside units on Interstate 64 , Virginia, USA ................. 31

9 Several on-board sensors in present-day vehicles. . . . . . . . . . . . 32

10 An example of on-board radar installed in a vehicle................ 34

11 Electronic stability control block diagram. .................. 34

12 Converting a GPS reading to the (Lane, Offset) form............ 36

13 Speed partitioned into ranges - saved in EDR $\ldots \ldots \ldots \ldots \ldots \ldots \ldots \ldots$

14 Illustrating communications between various entities in FRIEND. . . . . . 37

15 RFID readers in vehicles. . . . . . . . . . . . . 40

16 Illustrating the coverage area of a RSU $\ldots \ldots \ldots \ldots \ldots \ldots \ldots \ldots \ldots, 42$

17 A taxonomy of vehicular networks applications. . . . . . . . . . . . 46

$18 \quad$ Speed vs density. . . . . . . . . . . . . . . . . . . . . . . . . 53

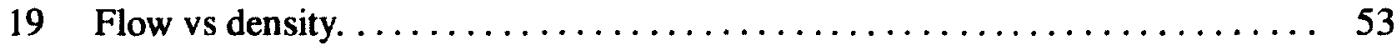

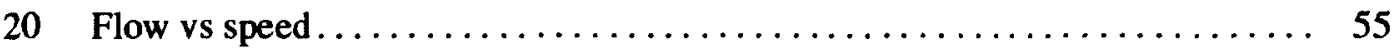

21 The pdf of headway distance versus the normal, log-normal, exponential and gamma distribution (From [4]) $\ldots \ldots \ldots \ldots \ldots \ldots \ldots \ldots \ldots \ldots \ldots, 57$ 
22 Headway simulation vs. Puan's field data. .................. 58

23 Illustrating the convolution $X=X_{1}+X_{2}+\cdots+X_{m} \ldots \ldots \ldots \ldots \ldots \ldots .58$

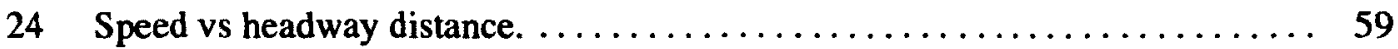

25 Feasible range for speed and headway distance. ................. 60

26 Number of clusters vs number of vehicles in $1 \mathrm{Km}, \ldots \ldots \ldots \ldots \ldots, 68$

27 Headway computation by RSUs. ..................... 69

28 Segment and RSU-RSU[i,j] definition $\ldots \ldots \ldots \ldots \ldots \ldots \ldots \ldots \ldots \ldots \ldots \ldots \ldots \ldots$

29 Illustrating non-time critical communication between adjacent RSUs. ...... 72

30 Illustrating the details of vehicle to RSU communication. ............ 74

31 Illustrating the headway distance buffer at $R S U_{i} \ldots \ldots \ldots \ldots \ldots \ldots \ldots$

32 Three different types of communications for SCEs. .............. 77

33 Packet format for data sent from SCE to RSU. ................. 78

34 RSU requesting information from SCEs in the direction of RSU-RSU[i,j] . . . 79

35 SCEs identifying the segment of an incident................... 80

36 SCEs exchanging data with smart wheels in vehicles............. 81

37 Illustrating the principle of locality. $\ldots \ldots \ldots \ldots \ldots \ldots \ldots \ldots \ldots \ldots \ldots \ldots \ldots$

38 Identifying the Head and Tail of a backup created from an incident on the

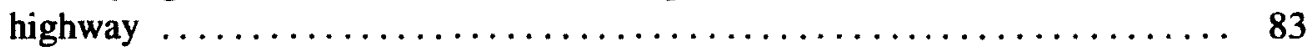

39 Illustrating the proof of Lemma $6.1 .4 \ldots \ldots \ldots \ldots \ldots \ldots \ldots \ldots \ldots$

40 Illustrating a two-level color-coded display of the state of traffic in FRIEND. . . 94

41 Illustrating the high-level four-state Markov chain defining color transitions in FRIEND. . . . . . . . . . . . . 94

42 Illustrating the internal 10-state Markov chain defining color transitions. .... 95

43 State diagram and relation with average headway distance and speed. . . . . 96

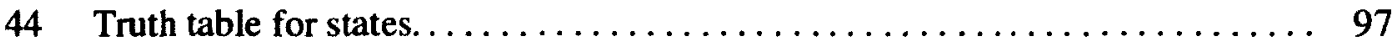


45 Transitions in the internal Markov chain. .................. 97

46 Highway incident classification. $\ldots \ldots \ldots \ldots \ldots \ldots \ldots \ldots \ldots \ldots \ldots$

47 Example of a moving incident on Interstate 64, Feb $2012 \ldots \ldots \ldots \ldots$

48 Different patterns of traffic in case of a blocking and non-blocking incident on highway. Blue lines represent the drivers who have changed lane. Black lines represent the drivers who passed on the pot-hole. .............. 101

49 Patterns of traffic in case of a moving incident on a highway. The change of

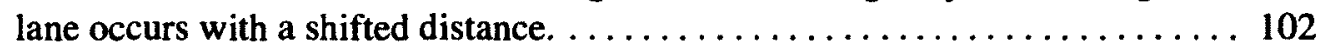

50 The two stages of incident dissemination in FRIEND $\ldots \ldots \ldots \ldots \ldots \ldots \ldots$

51 Illustrating Stage I of information dissemination. .............. 104

52 Illustrating Stage II of information dissemination in FRIEND. At $t=0$, incident just occurred, Head and Tail is identified. At $t=t_{1}$, vehicles started to move and Head is updated. At $t=t_{2}$, vehicles started to arrive in the Tail and

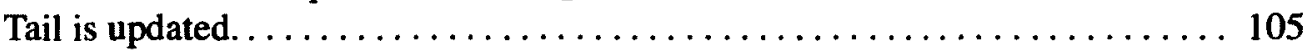

53 Illustrating the data exchanged in Stage II of information dissemination. . . . 106

54 Block diagram of FRIEND simulator parameters. $\ldots \ldots \ldots \ldots \ldots \ldots \ldots \ldots$

55 Illustrating vehicles color-mapped US13 highway in FRIEND . . . . . . . 109

56 Illustrating the percentage of message dropped as a function of traffic density. . 112

57 Simulation- Left A - Right B $\ldots \ldots \ldots \ldots \ldots \ldots \ldots \ldots \ldots \ldots \ldots \ldots \ldots \ldots$

58 Simulation- results - no sleeping mode. $\ldots \ldots \ldots \ldots \ldots \ldots \ldots \ldots \ldots \ldots$

59 Simulation- results - sleeping mode $\ldots \ldots \ldots \ldots \ldots \ldots \ldots \ldots \ldots \ldots \ldots \ldots$

60 Vehicle experiencing fog on the highway notifies other vehicles and the infras-

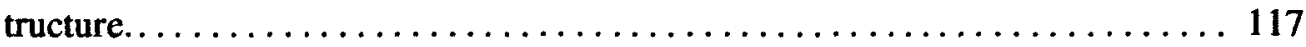




\section{CHAPTER 1}

\section{INTRODUCTION}

\subsection{MOTIVATION}

Several recent US Department of Transportation (US-DOT) and National Highway Traffic Safety Administration (NHTSA) statistics have revealed that in a single year congested highways cost the nation over $\$ 70$ billion in lost worker productivity and over 8.5 billion gallons of fuel wasted, not to mention high levels of carbon emissions [5, 6]. One important insight offered by these statistics is that over half of all congestion is caused by highway traffic-related incidents rather than by rush-hour traffic in big cities. Congested highways are the leading cause of traffic accidents, and projected data, extrapolated from January-September 2010 statistics (the most recent statistics available at this writing), predict for 2011 an estimated 38,000 traffic-related fatalities [6]. Figure 1 illustrates the principle sources of congestion from a recent US-DOT source [7].

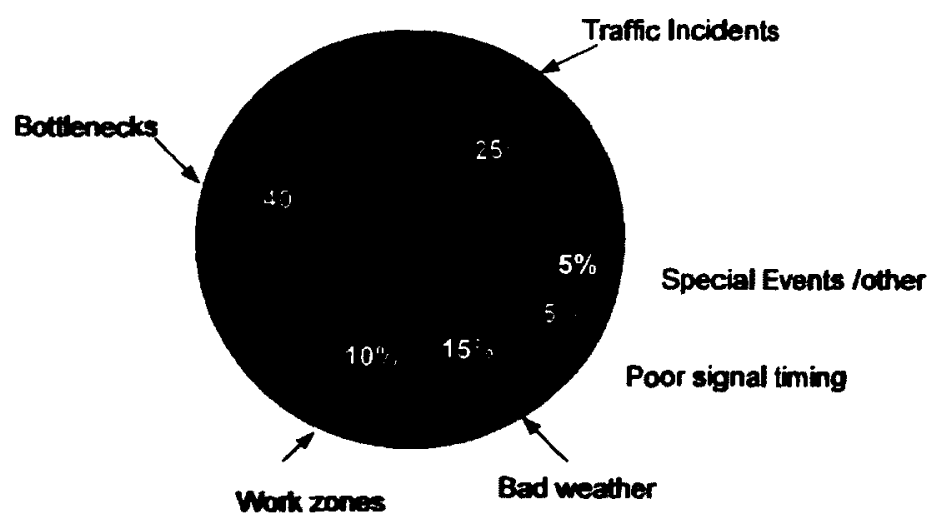

FIG. 1. Illustrating the main sources of congestion.

Unfortunately, on most US highways congestion is a common occurrence and, at the moment, advance notification of imminent congestion is unavailable [7, 8, 5]. It has been

This dissertation follows the style of The Physical Review 
argued convincingly that given sufficient advance notification, drivers could make educated decisions about taking alternate routes; in turn, this would improve traffic safety by reducing the severity of congestion reducing, at the same time, fuel consumption and carbon emissions $[9,10,11,12,13]$. In fact, reducing the number of traffic-related accidents, carbon emissions, fuel usage and travel delays on our roadways and city streets has been recognized as one of the National Grand Challenges [14, 15].

Traditionally, traffic monitoring was the purview of various federal and state transportation authorities. In support of providing traffic monitoring and data collection functions a series of methods and procedures, known collectively as Intelligent Transportation Systems (ITS) were set up over the decades. ITS uses mostly legacy technologies such as inductive loop detectors, magnetometers, video detection systems (e.g. cameras), acoustic tracking systems and microwave radar sensors in conjunction with probe vehicles and other means to estimate traffic parameters $[16,17,18,14]$. The estimated parameters are then aggregated at a central location (usually a Traffic Management Center) and used for various (mostly statistical) purposes. Up to very recently, the collected data and inferred traffic conditions were not shared with the travelling public. It is well documented that the hardware installed in support of collecting traffic-related data is expensive to install and costly to maintain and repair, making hardware-based traffic data collection and incident detection rather ineffective and inefficient [9]. Not surprisingly, the US-DOT has started to investigate a number of possible alternatives $[19,20,21]$. For example, in the next decade, the US-DOT plans to develop an architecture for vehicle infrastructure integration that will collect data from passing vehicles and, after aggregation at a central message switch, will be distributed to the traveling public [22]. The architecture document states that all messages will be digitally signed, with a central certificate authority responsible for distributing public and private encryption keys.

Even though wireless technology was available for the past 90 years, until very recently it was not used to enable communications in support of preventing, or mitigating the effect of, traffic-related events. All this has changed a decade or so ago with the advent of Vehicular Ad-hoc Networks (VANET) that employ a combination of Vehicle-to-Vehicle (V2V) and Vehicle-to-Infrastructure (V2I) communications intended to give drivers advance notification of traffic-related events. In V2V systems, each vehicle is responsible for inferring the presence of an incident based on reports from other vehicles. This invites a host of well-documented security attacks that could cause vehicles to make incorrect inferences, possibly resulting in increased traffic congestion and a higher chance of severe accidents 
$[23,24,25,26]$. In addition, because of their reliance on insecure V2V and V2I communications, most of the VANET systems proposed thus far have serious privacy problems. Indeed, because $\mathrm{V} 2 \mathrm{~V}$ communications can be traced back to individual vehicles, the driver of a vehicle will not be able to preserve their privacy and may be subject to impersonation or Sybil attacks. It was recently argued that even if several pseudonyms are used, detecting the true identity of the driver, and the attendant loss of privacy, appears hard to prevent $[27,28,29,30]$.

In support of traffic-related communications, the US Federal Communications Commission (FCC) has allocated $75 \mathrm{MHz}$ of spectrum in the 5.850 to $5.925 \mathrm{GHz}$ band specially allocated by the FCC for Dedicated Short Range Communications (DSRC) [31]. It was recently noticed that the DSRC spectrum set aside by the FCC, by far exceeds the needs of traffic-related safety applications [4]. The realization of this fact has already motivated the investigation of offering value-added services including on-line gaming, mobile infotainment, along with various location-specific services [32]. We fully expect third-party infrastructure providers to deploy various forms of road-side infrastructure as well as advanced in-vehicle resources such as embedded powerful computing and storage devices, cognitive radios and cognitive radio networks, and multi-modal programmable sensor nodes. As a result, in the near future, vehicles equipped with computing, communication and sensing capabilities will be organized into ubiquitous and pervasive networks with virtually unlimited Internet access while on the move. This will revolutionize the driving experience making it safer, more enjoyable, and more environmentally friendly.

In spite of the phenomenal advances in wireless technology, it was soon recognized that V2V and V2I communications, by themselves, do not suffice to prevent congestion and/or to mitigate its effect. The missing link is a tight integration, at several scales, of the capabilities of VANET and ITS. Not surprisingly, the past few years have seen a rapid converge of VANET and ITS leading to the emergence of Intelligent Vehicular Networks (InVeNet) with the expectation to revolutionize the way we drive by creating a safe, secure, and robust ubiquitous computing environment that will eventually pervade our highways and city streets. Lately, various solutions for traffic monitoring and incident detection have been proposed at the nexus of VANET and ITS.

However, the synergy of a confluence between VANET and ITS does not, in and by itself, address the most critical issues that underlie the deployment of automated incident detection and traffic-related information dissemination to the driving public. For example, the systems proposed thus far are neither secure nor privacy-aware, leaving the drivers 
vulnerable to location tracking, impersonation or Sybil attacks. The problem stems from the fact that, even with the use of pseudonyms, vehicular communications can be traced back to individual cars. As VANET is being integrated into the fabric of the society, secure networking is fundamental to achieve trustworthiness and effective operation in such a decentralized environment consisting of thousands of autonomous nodes with heterogeneous capabilities. We do not yet have a well-validated and widely-accepted set of design principles for building such systems.

Thus, there is a need for a secure and privacy-aware system that automatically detects existing traffic conditions and anticipates discernible trends in the traffic flow, based on which it can intelligently predict imminent traffic events and alert the driving public to their likely occurrence. Such a system, commonly referred to as a cyber-physical system (CPS), must integrate in a coherent way and at various scales the resources and capabilities of its hardware and the software components. A CPS can be thought of as a perfect example of the classical adage asserting that "The whole is more than the sum of its parts". Cyber-physical systems are characterized by a tight coordination between the system's computational and physical components. Today, we begin to see cyber-physical systems being developed in areas as diverse as the aerospace and automotive industries, nuclear power plants, civil engineering, health-care, transportation, entertainment, and consumer appliances. It is expected that the cyber-physical systems of tomorrow will far exceed those of today in terms of adaptability, autonomy, efficiency, functionality, reliability, safety, and usability [33].

\subsection{OBJECTIVES AND GOALS}

This thesis proposes to lay the theoretical foundations and to evaluate FRIEND: $A$ cyber-physical system for traffic-related event detection and dissemination. FRIEND will explore the integration of wireless networking with lightweight roadside infrastructure into a CPS that enables privacy-aware detection of traffic-related events and the dissemination to the driving public of such aggregated information both in the form of a color-coded traffic status report and traffic advisories in the case of serious incidents.

We are optimistic that FRIEND will improve road safety by alerting the public to discernible trends in traffic patterns and road conditions. FRIEND is not intended to supplant existing ITS systems. Instead, the intention is to show how existing ITS-based systems can be extended, along several dimensions, by integrating them with wireless communications and probabilistic reasoning. 
Put succinctly, FRIEND has three defining goals:

1. To collect traffic data about the traffic-flow;

2. To aggregate the collected data in a way that allows to detect and/or to anticipate traffic-related events;

3. To disseminate relevant traffic-related information to the driving public.

The workhorse of FRIEND is a set of smart cat's eyes and lightweight roadside units (RSUs) working in tandem. The roadside units, placed by the roadside, collect traffic related information from passing vehicles as well as from the neighboring smart cat's eyes. Suitable aggregated, the corresponding traffic-related information is then compared with a database of historical data in order to identify significant departures from expected values. Finally, relevant traffic-related information is disseminated to the traveling public in the form of a color-coded traffic status report and/or of traffic advisories alerting drivers to a variety of traffic-related events. Figure 2 shows the main infrastructure components of FRIEND.

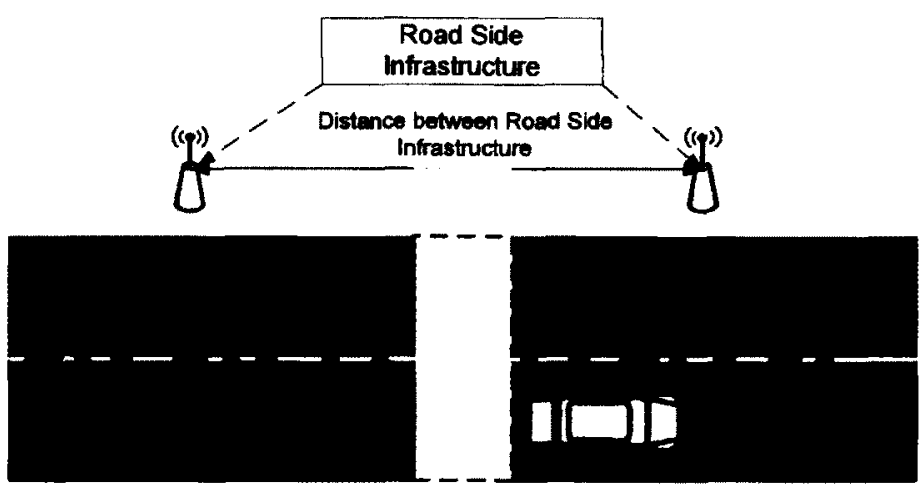

FIG. 2. Illustrating the main infrastructure components of the FRIEND.

FRIEND uses both V2I and Infrastructure-to-Infrastructure (I2I) communications. As discussed in detail in Chapter 3, the instances of V2I communications are

- Traffic data collection: As already mentioned, in FRIEND most of the traffic data is collected either by roadside units or cat's eyes from passing vehicles. It is important to note that the data collection is performed in a secure and privacyaware manner. In particular, each message is associated anonymously with a unique 
passing vehicle, so that no one vehicle can pretend to be multiple vehicles and, therefore, there is no need for vehicle IDs or pseudonyms;

- Traffic status dissemination: FRIEND disseminates to the drivers color-coded traffic status reports. These reports are location-aware, giving each driver a succinct synopsis of the status of the traffic up to ten $\mathrm{km}$ ahead;

- Traffic advisories dissemination: In the case of an incident, in addition to the color-coded traffic status report, FRIEND shares with the drivers information of the dynamics of the backup as well as the availability of alternate routes.

On the other hand, I2I communications are employed for the purpose of

- Acquiring coarse-grained incident location information: FRIEND has two mechanisms for locating an incident. In the first stage, a coarse-grain localization is performed by RSU to RSU communication, identifying an adjacent pair of RSUs that flank on both sides the location of the incident. The granularity of this localization is the distance between adjacent RSUs;

- Acquiring fine-grained incident location information: Once a coarse-grain localization of the incident has been performed, it is important to pinpoint the location of the incident, modulo the distance between a pair of adjacent cat's eyes. This phase is performed, essentially, by running a leader election protocol on the set of cat's eyes located in the road segment identified above;

- Acquiring fine-grained information about incident dynamics: It is fairly well known [18] that backups caused by incidents have complicated dynamics. It is of great interest to track, in near real-time, the location of the head and tail of the backup. This is done, again, by the cat's eyes that notice phase transitions between stopped and moving traffic or vice-versa.

\subsection{CONTRIBUTIONS}

The key technical contribution of this thesis is to propose FRIEND: a cyber-physical system for traffic-related event detection and dissemination. It is worth noting that the physical component of FRIEND employs suitably enhanced hardware already deployed along our roadways. These include the ubiquitous cat's eyes, the various lightweight infrastructure elements deployed by various transportation authorities as well as on-board 
sensors that are either already reality or will become so in the near future. One such device is the concept of a smart wheel capable of disseminating instantaneous speed information to the cat's eyes. While present-day vehicles do not feature smart wheels, they are technically feasible under current technology and, we feel, it is only a matter of time before they will be offered, as an option, in high-end vehicles.

Similarly, the cyber components of FRIEND are anchored in various probabilistic estimates of the interplay between speed and headway distance (intended to reflect traffic density). To the best of our knowledge, headway distance has not been used as an approximation of instantaneous traffic density. We show that even a small sample of such headway distances yield a fairly robust approximation of the instantaneous traffic density.

One of the interesting and novel contributions of FRIEND is the dissemination to the driving public of location-aware traffic status reports in the form of a color-coded synopsis. This allows drivers to obtain an up-to-the-minute view of the state of the traffic about ten $\mathrm{km}$ ahead of them. We anticipate that such information can be used judiciously by the drivers to avoid congested areas and, consequently, to help prevent and mitigate the effects of congestion on our roadways.

Yet another contribution is to disseminate to the drivers information of the status of the backup in the case of a serious incident. This is done by using the cat's eyes to keep track, in real time, of both the head and tail (to be defined in Chapter 6) of the congested area.

In summary, the key technical contributions of FRIEND are:

- Laying the theoretical foundations of a scalable, non-intrusive traffic-event detection strategy that is also privacy-aware;

- Exploring the architectural issues and design principles underlying FRIEND;

- Laying the theoretical foundations of detecting traffic-related events based on aggregating collected data; this will allow us to tailor the best information dissemination strategy - we will study formal models that distinguish between dissemination of time-critical and non-time-critical traffic-related events. To the best of our knowledge this is the first study of its kind;

- To the best of our knowledge, FRIEND is the first non-intrusive cyber-physical system that will be able to assist the authorities with managing traffic-related emergencies. 


\subsection{ROADMAP}

The remainder of this dissertation is organized as follows: in Chapter 2, we provide a succinct overview technical background, starting with related work pertaining to research to solve challenges in VANET, then discussing vehicle detection technology and then similar vehicular networking systems. In Chapter 3, we present the details of physical components of FRIEND, the main infrastructure used by our cyber-physical system, starting with nodes definition, then data communication in FRIEND and finally a taxonomy of applications in FRIEND. In Chapter 4, we reason about traffic flow parameters. In Chapter 5 , we present the details about protocols of communication between nodes in FRIEND. In Chapter 6, we discuss the decision making in FRIEND. In Chapter 7, we describe our simulation and evaluation. Finally, in Chapter 8 , we put the work in perspective, offer concluding remarks and highlight directions for future research. 


\section{CHAPTER 2}

\section{TECHNICAL BACKGROUND - REVIEW OF RELEVANT}

\section{LITERATURE}

FRIEND involves a number of research areas from wireless networking to intelligent transportation systems. The main goal of this chapter is to offer a succinct survey of the relevant state of the art in these areas, focusing particularly on previous work on which we plan to build.

In Section 2.1 we begin by discussing VANET Overview that motivated FRIEND in the first place. In Section 2.2, we summarize most of the vehicle detection technology. In Section 2.3, we discuss incident detection systems. In Section 2.4, we describe traffic monitoring in VANET. In Section 2.5, we discuss information dissemination systems. Finally, in Section 2.6, we give an example of one of VANET system "NOTICE".

\subsection{VANET OVERVIEW}

The original goal of VANET was to provide drivers with notification of real-time traffic conditions. A few of these systems have been developed and deployed in Japan [34] and Europe [35, 36] in partnership either with government agencies or automobile companies. One of the most notable US initiatives is the California Partners for Advanced Transit and Highways (PATH) program [37], which has investigated issues from automated driving to transportation modeling to driver warning systems. A typical VANET system for reporting traffic conditions consists of vehicles exchanging information about their position and speed with each other. The vehicles then use this information to determine where traffic slowdowns are occurring and report that information to other vehicles.

A VANET system for reporting traffic conditions can be implemented either using a purely ad-hoc network approach (i.e. V2V communications only) or using a network that includes roadside infrastructure (V2I). V2V systems $[38,39,40,41]$ are attractive because they require no additional infrastructure to be installed along the roadside. However, this lack of infrastructure can cause a problem in sparse traffic or at low market penetration rates where a critical mass of equipped vehicles may not exist. Additionally, V2V systems have known security issues and are susceptible to impersonation and Sybil attacks, where 
an adversary pretends to be one or more vehicles and sends erroneous data into the network $[42,43]$. V2I systems that rely on roadside infrastructure $[44,45,46,47]$ do so for various reasons, including aggregation, processing, and Internet or wider-area network access. A few approaches use the roadside infrastructure to provide encryption keys and pseudonyms in an attempt to protect driver privacy and provide secure communication. However, complex roadside infrastructure is expensive to deploy and consumes huge amounts of power [22].

\subsubsection{SECURITY AND PRIVACY ISSUES IN VANET}

The topology of VANET is highly dynamic, with vehicles joining and leaving, complicating authentication and the establishment of trust relationships between vehicles, inviting infiltration, impersonation and spoofing. The critical nature of the information propagated by VANET exacerbates security concerns. Thus, VANET are inherently vulnerable to a multitude of threats, including eavesdropping, physical tampering, infiltration, Denial of Service (DoS), spoofing, traffic analysis and impersonation, among many others.

The overwhelming majority of security countermeasures for VANET use cryptographic techniques. At the heart of virtually all of these solutions lie encryption key agreements between various entities designed to bootstrap trust between communicating vehicles or between vehicles and the roadside infrastructure. Recently, a number of protocols have been developed for key agreement supporting confidentiality and authentication services in VANET $[48,49,50,51,42]$. There seems to be a consensus that while symmetric-key-based protocols tend to be simpler, some of these protocols are not sufficiently flexible for use in dynamically re-configurable networks, since they cannot efficiently handle unanticipated additions of vehicles to the network. Public key-based key establishment protocols offer more flexibility and scalability. But, the public key algorithms used in these protocols tend to be slow and to consume a great deal of computational resources.

A fundamental problem in securing V2V communications is authentication. One of the main authentication problems is broadcast authentication, which involves verifying the source of broadcast transmission to multiple receivers. The task of providing broadcast authentication in VANET is non-trivial. For example, public-key-based digital signatures typically used for broadcast authentication, are too expensive to be used in VANET where reconfiguration is the norm rather than the exception. Also, symmetric-key-based mechanisms cannot be directly applied to broadcast authentication, since a compromised receiver 
can then easily forge any message from the sender.

In VANET there is a strong correlation between a vehicle's identity and that of the driver. It follows that any effort to protect driver privacy must attempt to make the link between the two harder to detect. Because of the large number of vehicles on our roadways and city streets and of the sophistication of possible attacks, privacy protection must be both robust and scalable.

Often, ensuring security means sacrificing privacy because valid identification of the vehicles is needed to verify digital signatures for authentication. Once a vehicle's identity is established it can be easily tracked, raising serious privacy concerns. For instance, most people would be uncomfortable with the notion that a traffic monitoring system could be used to track their movements over a period of time.

To avoid easy identification of vehicles while facilitating authentication, several approaches advocate the use of pseudonyms that change at various times, according to a particular set of rules $[52,53,54,49]$. To achieve this, vehicles need to either contact roadside infrastructure to obtain new pseudonyms periodically, contact a certificate authority to obtain a new key each day, or preload many pseudonyms into a tamper-proof device in the vehicle $[51,55]$. The presence of many pseudonyms in one vehicle could make the system susceptible to Sybil attacks. Other approaches use roadside infrastructure for re-anonymization or require that the vehicle be in contact with a certificate authority [43]. Even with the use of pseudonyms, it has been argued that tracing the pseudonym back to the original owner of the vehicle is difficult to prevent $[56,55]$.

V2V and V2I communications have been contemplated as a means towards Cooperative Collision Warning Systems (CCWS) that use a combination of V2V and V2I communications to allow drivers to alert each other to possible collision events $[57,58,59]$. While the proposed systems seem promising, and some aspects have been adopted in Automatic Cruise Control Systems, their large-scale deployment is hampered by numerous security and privacy issues $[57,9,60]$.

Yet another line along which traditional ITS is augmented is automatic vehicle identification (AVI), automatic vehicle location (AVL) and other wireless location technologies (WLT) [9]. However, these technique are in their infancy and are afflicted with known privacy and security problems that are likely to delay or even derail their widespread use.

Vehicles in FRIEND can act as data mules, carrying encrypted messages between adjacent roadside units. The novelty of FRIEND lies mostly in the symbiotic relationship between the roadside infrastructure and cat's eyes. 
Directional antennas have been proposed for use in ad hoc networks to reduce interference by allowing nodes to receive signals from only a certain direction $[61,62,63]$. Recent work [64] has applied this idea to VANET to allow high priority messages (e.g. from emergency vehicles) to have contention-free access to the medium. In addition to controlled flooding techniques, we will investigate the use of directional antennas to keep message communications with a single vehicle within a single lane of traffic.

\subsection{VEHICLE DETECTION TECHNOLOGY}

Intelligent Transportation Systems (ITS) have traditionally provided traffic monitoring and data collection functions using various vehicle detection technologies. In the past decade or so, we have witnessed an unmistakable confluence between VANET and ITS systems. We expect that in the near future some of the vehicle detection technologies will merge into very powerful on-board capabilities.

The US-DOT plans to develop in the next decade an architecture for vehicle infrastructure integration [22]. This architecture relies on a heavy networked roadside infrastructure that will collect data from passing vehicles and will relay it to a central message switch responsible for distributing the data to interested parties. The architecture document states that all messages will be digitally signed, with a central certificate authority responsible for distributing public and private encryption keys.

In order to turn traffic data obtained through the methods described above into incident detection data, automatic incident detection (AID) algorithms are used. There has been much research in ITS devoted to various types of AID algorithms $[65,66,67,68,69,70$, 71]. One of the most used is the McMaster algorithm, which compares current traffic flow to a model of expected flow. The Federal Highway Administration (FHA) reports that as much as $90 \%$ of traffic incidents are currently reported by citizens through 911 calls, which outperforms any of the existing AID algorithms [14]. The key in these situations is delivering the information to traffic managers, verifying the incidents, and finally disseminating the information to the driving public.

Quite recently, cell phone technology has been proposed for traffic monitoring in limited deployments [72]. The current state-of-the-art is a handoff based system that tracks the cellular handoffs between towers using handoff information already collected by the cell phone companies. These systems use elapsed times between handoffs to determine link speeds. This technology is much like that used for probe vehicles, but there is no special equipment needed in the vehicle, besides a cell phone. According to a study from 
the University of Virginia [73], these cell phone monitoring systems still have challenges to overcome, such as accuracy in determining vehicle position from the cell phone signal, matching the location to a physical roadway, and overcoming the small numbers of callers that can serve as probes. Another problem is that tracking cell phone movements raises privacy concerns, even though assurances have been made that the information is not tied to a specific cell phone and that traffic tickets will not be issued using this system.

We note that many of the proposed ITS approaches that supplement ILDs and video cameras by the use of wireless communications have raised security and privacy concerns, very similar to those in VANET.

We now review some of the most popular non-intrusive vehicle detectors. There are some factors that affect the choice of the sensor type such as the type of detection, traffic flow, sensor capability, weather conditions and construction conditions.

\subsubsection{INDUCTIVE LOOP DETECTORS (ILD)}

ILDs are the most commonly used sensors in traffic surveillance and management applications. Currently, most incident detection systems and algorithms use traffic data derived from ILDs. The standard ILD is a length of insulated wire bent into a closed shape, traditionally a square or a rectangle, and connected to a power source/sensor on both sides of the wire. The wire loops are embedded in a shallow cutout in the pavement. A lead-in cable runs from a roadside pull box to the controller cabinet to an electronics unit located in the controller cabinet. When a vehicle stops on or passes over the loop, the inductance of the loop decreases, which in turn, increases the oscillation frequency and causes the electronics unit to send a pulse to the controller, indicating the passage of a vehicle and registering its presence in its detection zone. New versions of ILDs use higher frequencies to identify specific metal components of vehicles, which can be used to classify vehicles $[2,74]$.

The sensitivity of an ILD is adjustable and can be tuned for a variety of different locations and environments. In operation, ILDs tends to go out of tune over time and requires readjustment. The received presence information can be used to calculate volume and occupancy. Occupancy is computed by taking the ratio of time the detector registers the presence of vehicles in its detection zone to the total sample time. However, ILDs have a tendency to double-count trucks $[2,75]$. Due to the vehicle's structure, trucks as well as other long vehicles often are regarded as two passenger cars by an ILD. Tractor-trailer units often have concentrations of metal far enough above the loop so that the detector 
electronics cannot detect them, resulting in detection gaps. ILD systems still suffer from poor reliability, related to causes such as inclement weather, improper connections made in pull boxes, and in the application of sealants over the cutout. These problems are accentuated when ILDs are installed in poor pavement or in areas where utilities frequently disturb the roadbed. Most cities with mature systems report that 25 to 30 percent of their detectors are not operating properly at any given time. Moreover, the installation and maintenance of ILDs require lane closures to dig grooves in the road, causing traffic disturbances. In addition, the precise nature of an incident detected by ILDs cannot be ascertained, and ILDs perform less effectively for incident detection in low volume conditions $[2,76]$.

\subsubsection{MAGNETIC SENSORS - PASSIVE AND ACTIVE}

Magnetic sensors work on the principle that the presence of a vehicle distorts the magnetic field which shrouds the earth. Although different in appearance and specific technology, they operate on a similar principle to ILDs $[2,74]$. Magnetic sensors are often installed in place of loops on bridge decks, and in heavily reinforced pavement, where steel adversely affects loop performance [74]. ILDs and magnetic sensors each have their respective applications and tend to complement one another. There are two types of magnetic sensors for traffic flow parameter measurement: active devices, such as magnetometers; and passive devices.

The first type, two-axis fluxgate magnetometers, are active devices, excited by an electrical current in windings around a magnetic core material. They detect changes in the vertical and horizontal components of the earth's magnetic field. They can measure the passage of a vehicle when operated in the pulse output mode, yielding count data, and provide a continuous output as long as a vehicle occupies the detection zone when operated in the presence output mode. The Self-Powered Vehicle Detector (SPVD), a type of magnetometer developed with FHWA support, is powered by a self-contained battery, with a limited expected life, 1 to 2 years. It is connected to a remotely located controller cabinet via a radio link. Thus, no direct connection is required $[2,74]$.

A magnetometer presents installation and maintenance problems similar to ILDs. To install and repair a magnetometer, traffic needs to be disrupted for a sufficiently long period for removing the sensor and reinserting it in a borehole. Compared to an ILD, this device, though road-embedded, shortens lane closure time for each repair, but increases the frequency of lane closures for such repairs, especially in the case of SPVD $[2,75]$. 
The second type, passive magnetic detectors, sense perturbations in the earth's magnetic flux produced when a moving vehicle passes over the detection zone. These magnetic sensors are induction magnetometers. Most require some minimum vehicle speed for producing an output signal, usually 3 to $5 \mathrm{mph}$; hence, they cannot detect stopped vehicles nor provide presence measurements $[77,78,76]$.

Magnetic detectors are easier to install and more maintainable than ILDs for a similar price [79]. They can come pre-installed in tubing. Compared to ILDs, magnetic detectors can sustain greater stresses and break down less often. Alternative installation procedures may further improve their reliability. The biggest disadvantage of magnetic detectors is that they cannot measure occupancy. Speed may be calculated by installing two magnetic detectors in a close succession, and from speed and flow measurements, occupancy can be calculated. However, if two magnetic detectors are placed too closely together, they may interfere with each other $[2,75]$.

\subsubsection{ULTRASOUND SENSORS}

Ultrasonic sensors transmit pressure waves of sound energy at frequencies between 25 and $50 \mathrm{KHz}[2,75,74]$. They fall into two types: pulse-waveform ultrasonic sensors and constant frequency ultrasonic sensors. Most ultrasonic sensors operate with pulse waveforms; only this type is discussed here. Pulse waveforms are used to measure distances to the road surface and the vehicle surface by detecting the portion of the transmitted energy that is reflected back towards the sensor. When a distance other than that to the background road surface is measured, the sensor interprets that measurement as the presence of a vehicle. The received ultrasonic signal is converted into electrical energy that is analyzed by signal processing electronics. This technique is similar to that used by pulse microwave sensors.

Ultrasonic sensors can measure speed, occupancy, presence, and in some configurations, queue length. Moreover, vehicle profiling can be achieved by installing a pulse ultrasonic detector above the roadway; excellent classification performance can be achieved for most vehicle types. Ultrasonic sensors have no moving parts so they tend to be reliable, durable and require little maintenance. They are also small and can be sited permanently or used as a portable unit. However, air turbulence and temperature adversely affect operational performance [2].

\subsubsection{INFRARED SENSORS}


The infrared sensors referred to here are non-image infrared devices. Infrared sensors can operate in active or passive modes. Similar to microwave sensors, infrared sensors are mounted overhead or in a side-looking configuration. In the active mode, a detection zone is illuminated with infrared energy transmitted from laser diodes operating in the near infrared spectrum. A portion of the transmitted energy is reflected back to the sensor by vehicles traveling through the detection zone. An infrared-sensitive element converts the reflected energy into electrical signals that are analyzed in real time $[2,76]$. Infrared sensors can measure presence, speed, volume, occupancy, and vehicle classification. Active infrared detectors are vulnerable to weather conditions such as fog, clouds, shadows, mist, rain, and snow, which scatter and attenuate wave energy. High cost is cited as one of the reasons that they are not more widely used in traffic surveillance. Active sensors are more expensive than passive ones. Passive infrared detectors measure the same traffic parameters as active detectors except for speed. They do not transmit their own energy but use an energy-sensitive element to measure the thermal energy (i.e., temperature) emitted by vehicles, which differs from the energy emitted from the road, in the field of view of the detector. When a vehicle enters the field of view, the change in emitted energy from the scene is sensed. Passive infrared sensors have difficulty measuring speed because the extended nature of the vehicle distorts the infrared signature, making velocity less clear. On the other hand, multi-zone passive infrared sensors can measure speed and vehicle length as well as the more conventional vehicle count and lane occupancy $[2,78]$. Inclement weather, such as fog, snow, and precipitation that scatter energy, and changes in light, may have adverse effects on performance.

\subsubsection{MICROWAVE SENSORS}

Microwave sensors currently used in traffic surveillance fall into two types in terms of their working waveforms: constant-frequency waveform $(\mathrm{CW})$ and frequency-modulated waveform (FMCW). The first type, termed the continuous microwave detector, makes use of the Doppler principle to compute vehicle speed from $\mathrm{CW}$ microwave radar that transmits electromagnetic energy at a constant frequency. Because only moving vehicles are detected by $\mathrm{CW}$ Doppler radar, vehicle presence cannot be measured with this waveform and hence this type of microwave sensor is not suitable for incident detection. The second type is termed the pulse microwave detector. These detectors transmit electromagnetic energy in frequency bands between 2.5 to $24.0 \mathrm{GHz}$. They are capable of counting vehicles, measuring speeds and detecting vehicle presence. Pulse microwave detectors can 
also classify vehicles by measuring the vertical profile of a vehicle [2].

Microwave sensors provide a cost-effective alternative to ILDs for vehicle presence detection and hence for incident detection. They are relatively smaller, lighter in weight and easier to install than ILDs and magnetic sensors, and they can detect multi-lane traffic and cover a longer range (say 100 meters to 1000 meters). Their small size, low cost and low power consumption makes them suitable for traffic surveillance both at intersections and on highways. However, it needs to be noted that that a newly installed microwave sensor may interfere with other similar microwave-based devices in its vicinity.

\subsubsection{ACOUSTIC SENSORS}

Acoustic sensors are operated in passive mode and are usually configured as a two dimensional dipole array of microphones that are sensitive to the acoustic energy (i.e., audible sounds) produced by approaching vehicles. The time delay between the arrival of sound at the upper and lower microphones changes with time as the vehicle emitting the sound passes under it. When a vehicle passes through the detection zone, an increase in sound energy is detected by the signal processing algorithm and a vehicle presence signal is generated. When the vehicle leaves the detection zone, the sound energy level drops below the detection threshold and the vehicle presence signal is terminated. Vehicles are tracked using cross-correlation between microphones. Best results are achieved when the data is filtered to a bandwidth of $50-2000 \mathrm{~Hz}$.

For this type of acoustic sensor, the preferred mounting is at a 10- to 30-degree angle from the vertical direction. This sensor can count vehicles and measure presence, speed, volume and occupancy. Interference between the noises of multiple vehicles is a limitation to acoustic technology. Its performance is also affected by low temperature and by snow, and dense fog that may muffle sound and lead to under-counting. A second type of acoustic sensor uses a fully populated microphone array and adaptive spatial processing to form multiple detection zones. This sensor can monitor as many as six to seven lanes when mounted over the center of the roadway. Mounting heights range from 20 and 40 feet [2].

\subsubsection{LASER SENSORS}

Laser sensors operate in active mode and work on the same principle as microwave radar sensors, using light frequencies. Laser sensors can offer high-speed measurement accuracy and measure all the vehicle characteristics needed for traffic surveillance and incident detection. A vehicle detection and classification system utilizing laser sensors has 
been deployed on Interstate 4 in Orlando, Florida, for obtaining data needed for incident detection. Generally, laser sensors are mounted on a gantry over the highway; each unit can provide coverage for two adjacent lanes. A wireless modem connected with the sensor transmits the information between the sensor and a control and processing computer. Almost all traffic parameters, such as presence, classification, speed, volume, occupancy and so on can be measured by laser sensors. Moreover, they provide the detailed vehicle shape characteristics needed to uniquely identify vehicles. This capability can be used to measure travel times between two locations on highways, which offers the possibility to develop incident detection schemes based on variations in travel time like the ones that utilize probe-based data $[2,80]$.

\subsubsection{VIDEO IMAGE PROCESSORS}

Video image processors (VIP) employ machine vision techniques to automatically analyze traffic data collected with Closed Circuit Television (CCTV) systems or other video cameras. A VIP system consists of one or more video cameras, a microprocessor based computer for digitizing and processing the video imagery, and software for interpreting the images and converting them into traffic flow data. The image processing algorithms in the computer analyze the variation of groups of pixels contained in the video image frames. By analyzing successive video frames, the VIP is capable of calculating traffic flow information.

VIP systems fall into one of three classes: tripline, closed-loop tracking, and data association tracking $[2,74]$. Tripline systems operate by allowing the user to define a limited number of detection zones in the field of view of video cameras. These systems are the most common and are essentially expensive loop emulators. Closed-loop tracking systems permit vehicle detection along larger roadway sections, which provide additional traffic flow information such as lane-to-lane vehicle movements. Data association tracking systems can identify and track a specific vehicle or group of vehicles as they pass through the field of view of the camera, in which the unique connected areas of pixels are searched, identified and tracked from frame-to-frame to produce tracking data for a selected vehicle or vehicle group. This technique has the potential to provide link travel time and origindestination pair information $[2,81]$.

One of the primary advantages of using VIP for incident detection is that incidents are not blocked by the resultant traffic queues if the surveillance video camera is installed so as to provide upstream viewing $[2,74]$. Some VIP systems are able to exact a wide 
range of traffic parameters, including density, queuing length and speed profiles. Other advantages of using VIP for incident detection also include possibly short detection time, quick identification, as well as recognition of the incident type (using human operators), multi-lane surveillance by one sensor and easy installation. The performance of VIP systems, however, is affected by variations of light and climate, so the installation position and the calibration of image processing algorithms need to be adjusted accurately. In addition, the transmission of video images requires more bandwidth than transmission of voice and data, which increases the cost of transmission. There appear to be no technological barriers, given the technical maturity of VIPs, to the implementation of incident detection systems; the main challenge lies in refining its corresponding automatic incident detection algorithms $[2,81]$.

\subsection{INCIDENT DETECTION}

Various incident detection techniques have been proposed in the past decades. The goal of these techniques is to automatically identify the existence of highway incidents and their location. We divide these systems into different categories. This subsection is devoted surveying different incident detection mechanisms proposed in the literature. In doing so, our goal is to clarify the advantages and disadvantages of each system. Incident detection systems can be classified into several groups based on the data collection technologies employed and on the data processing algorithms used. Variations in sensor-and-algorithm schemes result in a variety of solutions for incident detection [2]. For example, Parkany [2] classifies incident detection algorithm into roadway-based, probe-based and driver-based incident detection technologies. The corresponding algorithms are reviewed and evaluated in detail.

Pucher et al. [82], the authors present a detection and tracking methods for highway monitoring based on video and audio sensors as well as various combinations of these two modalities. The main disadvantage of these techniques is the bad performance in case of some weather conditions such as fog, storm, or heavy rain or poor visibility. We refer to Figure 3 for an example of a general audio/video highway monitoring system.

Existing incident detection systems measure traffic parameters such as flow, speed and density and the data is sent, on a regular basis, to Traffic Management Centers (TMC) 


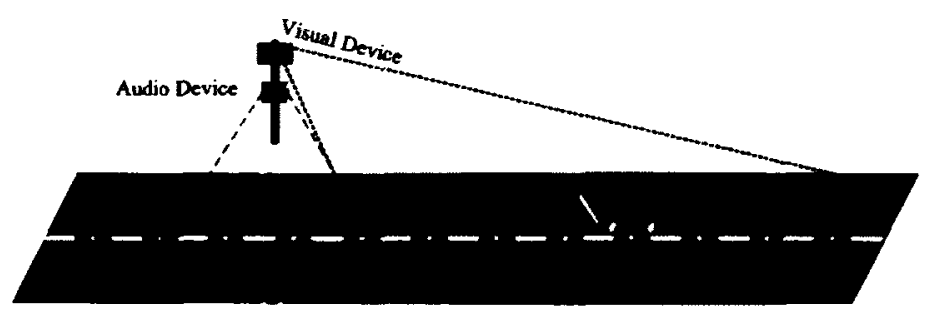

FIG. 3. Example of audio/video highway monitoring system

for aggregation and decision making. Table 1 gives a brief description of different detection/sensor technologies that have been used [2].

TABLE 1. A sample of detection/sensor technologies.

\begin{tabular}{|c|c|}
\hline \multicolumn{2}{|c|}{ Vehicle detection technologies } \\
\hline Roadway-based sensors & $\begin{array}{l}\text { Inductive loop detectors (ILD) } \\
\text { Magnetic sensors } \\
\text { Microwave sensors } \\
\text { Infrared sensors } \\
\text { Ultrasonic sensors } \\
\text { Acoustic sensors } \\
\text { Laser sensors } \\
\text { Video image processors }\end{array}$ \\
\hline Probe-based sensors & $\begin{array}{l}\text { Automatic vehicle location (GPS) } \\
\text { Signpost/beacon system } \\
\text { Cellular geolocation system } \\
\text { Automatic vehicle identification }\end{array}$ \\
\hline Driver-based sensors & $\begin{array}{l}\text { Highway service patrol } \\
\text { Remote CCTV monitoring } \\
\text { Cellular phone reports }\end{array}$ \\
\hline
\end{tabular}

All these sensors including ILDs, magnetic sensors, microwave sensors, infrared sensors, ultrasonic sensors, acoustic sensors, laser sensors and video image processors have been used as roadway-based sensors. Some of these sensors have been installed in the pavement, while others have been installed on the side of the road. Researchers have used a modified techniques to combine sensors [83], where magnetic sensors were used for detection with an optical wake-up method. Probe-based sensors are used in vehicles using GPS [84]. Driver-based sensors such as cellular phones detect $38 \%$ of the incidents, and 
$1 \%$ of the other events. This is probably because incidents (having blocked travel lanes and impeded traffic flow) are likely to get immediate attention from users [85].

Most of the incident detection techniques use pattern recognition algorithms. The DT algorithms, or so-called California algorithms, are the most widely known comparative algorithms. This type of algorithm is based on the principle that an incident is likely to cause a significant increase in upstream occupancy while simultaneously reducing occupancy downstream [2]. Subramaniam et al [1], classified incident detection algorithms into 5 categories: pattern recognition, statistical processing, catastrophe theory, neural networks and video image processing, as shown in Table 2 [2].

TABLE 2. Performance comparison of several incident detection algorithms based on ILD and VIP sensors, source $[1,2]$.

\begin{tabular}{|c|c|c|c|c|}
\hline \multicolumn{5}{|c|}{ A taxonomy of incident detection algorithms. } \\
\hline Type & Algorithm & $\begin{array}{l}\text { Detection } \\
\text { Rate }\end{array}$ & $\begin{array}{l}\text { False } \\
\text { Alarm } \\
\text { Rate } \\
\end{array}$ & $\begin{array}{l}\text { Mean } \\
\text { time to } \\
\text { Detect }\end{array}$ \\
\hline Pattern recognition & $\begin{array}{l}\text { California algorithm\#7 } \\
\text { APID algorithm }\end{array}$ & $\begin{array}{l}67 \% \\
86 \%\end{array}$ & $\begin{array}{l}0.13 \% \\
0.05 \%\end{array}$ & $\begin{array}{l}2.91 \mathrm{~min} \\
2.55 \mathrm{~min}\end{array}$ \\
\hline Statistical processing & $\begin{array}{l}\text { SND model } \\
\text { Bayesian algorithm } \\
\text { ARIMA model } \\
\text { Smoothing model } \\
\text { DES model } \\
\text { Filtering model }\end{array}$ & $\begin{array}{l}92 \% \\
100 \% \\
100 \% \\
92 \% \\
82 \% \\
95 \% \\
\end{array}$ & $\begin{array}{l}1.3 \% \\
0 \% \\
1.4 \%- \\
2.6 \% \\
1.87 \% \\
0.28 \% \\
1.5 \% \\
\end{array}$ & $\begin{array}{l}1.10 \mathrm{~min} \\
3.90 \mathrm{~min} \\
0.39 \mathrm{~min} \\
0.74 \mathrm{~min} \\
5.05 \mathrm{~min} \\
0.67 \mathrm{~min}\end{array}$ \\
\hline Catastrophe theory & McMaster algorithm & $100 \%$ & $0.04 \%$ & $1.5 \mathrm{~min}$ \\
\hline Artificial intelligence & ANN model & $97 \%$ & $0.21 \%$ & $2.83 \mathrm{~min}$ \\
\hline Video image processing & $\begin{array}{l}\text { INVAID-TRISTAR } \\
\text { system }\end{array}$ & $90 \%$ & $\begin{array}{l}1 \text { every } \\
3 \text { hours }\end{array}$ & $0.33 \mathrm{~min}$ \\
\hline
\end{tabular}

As can be seen from the Table 2, the detection rate of the Bayesian, ARIMA and McMaster algorithms is the highest, as shown in Figure 4. FRIEND runs a Bayesian algorithm which increases the detection rate. Moreover, the false alarm rate of Bayesian 


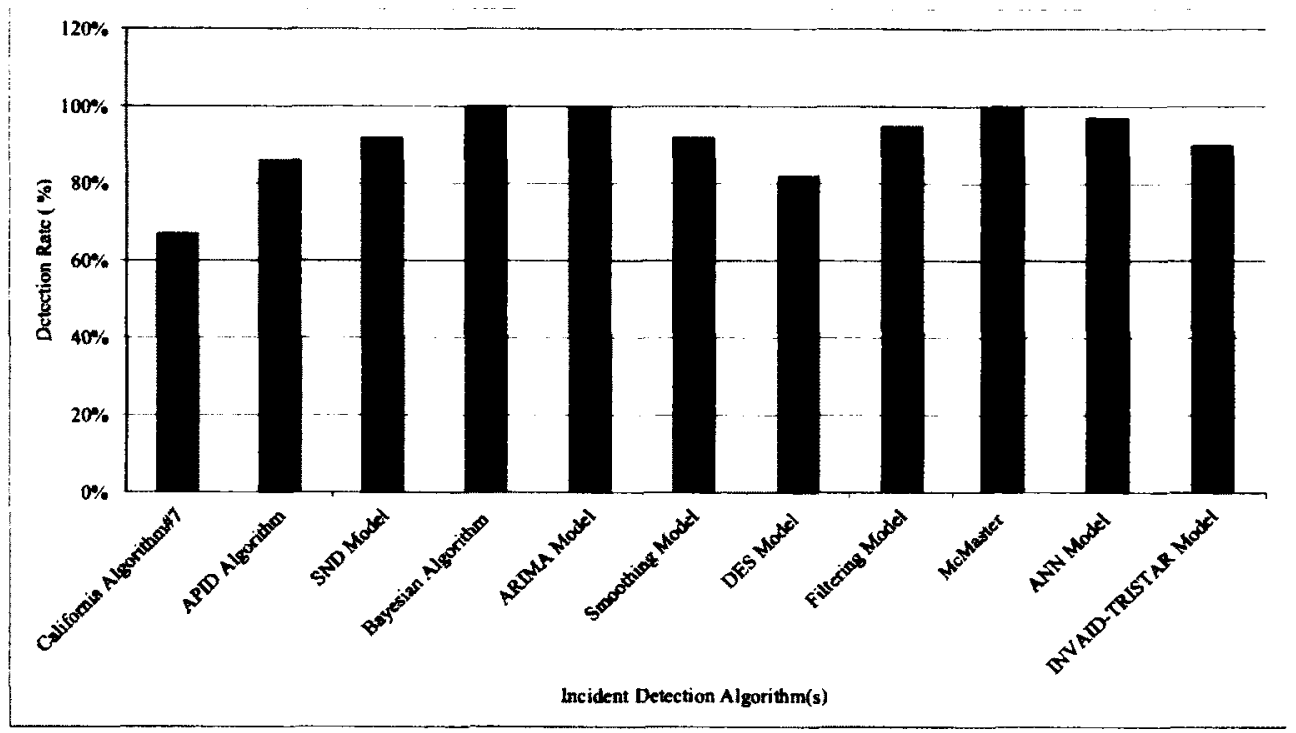

FIG. 4. Comparison of detection rates of several incident detection algorithms.

algorithms is almost $0 \%$ as shown in Figure 5. We note that both Figures 4 and 5 were created using data from [2] and [1]. However, it is the case that the Bayesian algorithms take more time on the average to detect an incident.

\subsection{TRAFFIC MONITORING IN VANET}

Recent advances in wireless sensor and networking technologies have suggested innovative and more cost-effective alternatives which employ a combination of V2V and V2I wireless communication to provide novel solutions for traffic monitoring and incident detection [20] in ITS. Along similar lines, work in transportation engineering has resulted in the development of a cement-based piezoelectric sensor [86] that can be used to detect passing vehicles. In this work, a piezoelectric ceramic plate was placed between two cubes of hardened cement sand paste. This sensor can then be embedded in the roadway and perform detection similar to inductive loops. These sensors are durable, do not corrode, cannot be damaged by thermal expansion of the road, and can be made of inexpensive materials. This work has been the basis of NOTICE [87, 88, 89]. Specifically, the NOTICE system involves embedding intelligent sensor belts in roadways and using 


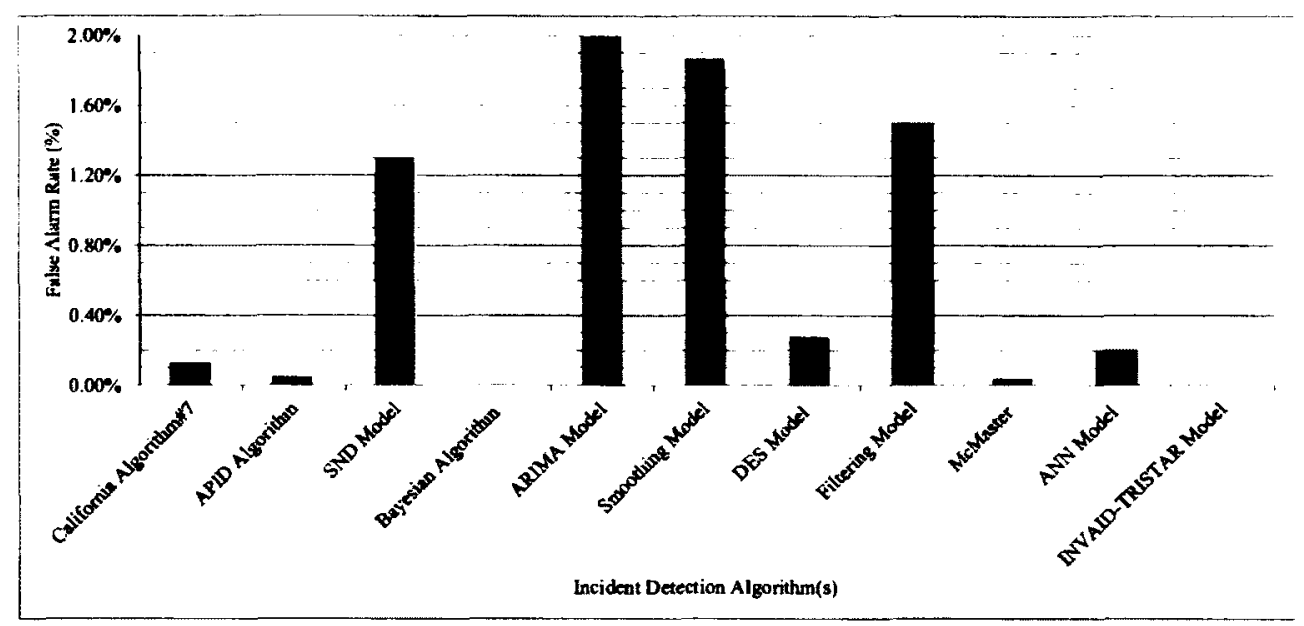

FIG. 5. Comparison of false alarm rates of several incident detection algorithms.

these belts to detect traffic incidents ranging from congestion to lane obstructions and potholes. In this regard, NOTICE has a great deal in common with ILDs since both systems are intrusive and by virtue of cutting in the surface of the roads, contribute to weakening the structural integrity of roadways. Extrapolating from past experience with ILDs [90], sensor belts embedded in the roadway are very likely to suffer from reliability problems and to contribute to the creation of potholes.

The main goal of most VANET systems is to communicate information as quickly as possible to as many vehicles as possible, and data dissemination is an important part of any VANET system. Most systems use broadcast mode to disseminate the information. When broadcast is used by many nodes, care has to be taken that messages are not sent unnecessarily and that nodes do not try to send all at the same time, creating contention for the wireless medium. In these cases, strategies for intelligent flooding should be used. There have been several approaches to controlled flooding within multicast groups based on node location and travel direction [91, 92, 93, 94]. Harras et al. [95] present an evaluation of several different controlled flooding schemes. All of these techniques are targeted towards highly-mobile, sparsely-connected networks, of which VANET are a fitting example, especially during early phases of system deployment.

Another method of disseminating information in a VANET, or any sparsely populated network, is by using data mules [96]. Data mules are mobile nodes that buffer and carry data from one place in the network to another. The CarTel project [97] uses vehicles both as probes to collect data about travel times and as data mules to deliver information to the 
system's central server.

\subsection{INFORMATION DISSEMINATION SYSTEMS}

Information dissemination, also known as data dissemination, is a critical component of vehicular networks. A good deal of contemporary VANET and ITS research focuses on preventing secondary accidents [98] or notifying drivers far away from the accident of an expected delay that gives them the option to continue or to exit before reaching the location of the incident. Abuelela et al. [87] proposed NOTICE, a secure architecture for notification of traffic incidents. NOTICE is built around the idea of embedding sensor belts in the roadway at regular intervals, avoiding the heavy cost of optical fiber that has been used in ILDs. NOTICE depends on vehicles forwarding the messages, which can suffer from disconnection problems in sparse traffic [99]. In addition, because of the need to embed sensor belts in the road itself, NOTICE is an intrusive system that is likely to be costly to maintain and may also contribute to the weakening of the road. In addition, the current version of NOTICE cannot solve the problem of blocking incidents, that is, incidents that occlude the road completely.

Little et al. [100] proposed an information propagation scheme that assume very close directions of a highway. Maintaining clusters is an overhead for Little's system. Xeros et al. [101] introduced a system for information propagation at intersections. Hafeez et al. [102] proposed a model for radio propagation in cities that takes into account all the obstacles. Wu et al. [103] used vehicle-to-vehicle communication to propagate information on highways. Nadeem et al. [104] made a comparative study of data dissemination models for VANET. Their study showed that the main problem in these systems is that they require expensive infrastructures to be installed on every road in which the system is going to be used. Additionally, they are not scalable owing to their centralized design.

The main disadvantage of current dissemination methods is that they cannot handle disconnection problems, especially with sparse traffic such in GVGrid, MURU and PBR, defined in Table 3. In FRIEND, we depend on both vehicles and infrastructure, which allows us to disseminate information without suffering from disconnecting problems.

\subsection{NOTICE: AN ARCHITECTURE FOR THE NOTIFICATION OF TRAFFIC INCIDENTS}


TABLE 3. Examples of some information dissemination algorithms.

\begin{tabular}{|c||c|}
\hline Technique & Functions \\
\hline GVGrid & On-demand, position-based routing protocol \\
\hline MURU & Multi-hop routing protocol \\
\hline PBR & Position based routing \\
\hline
\end{tabular}

In this section, we discuss NOTICE $[87,105,88,106]$, which is an architecture for the notification of traffic incidents on highways. We begin with automatic incident detection (deterministic) technique in NOTICE, then we discuss their probabilistic technique for detecting permanent and temporary incidents in VANETs [106].

NOTICE, Abuelela et al. [87] relies on sensor belts embedded in the roadway every couple of kilometers. Each belt is responsible for collecting and managing data from vehicles passing over the belt. Collectively, the belts maintain the collected data in a table called RoadImage. The table collect the number of vehicles that have passed over the belt in a specific lane. Their idea is that a negative peak in the row corresponding to the lane contains an incident. To detect whether an incident has occurred or not, NOTICE computes the average and standard deviation for the row and finds the minimum. Then, uses the idea of bandpass filter to take away any oscillation or incorrect values. Finally, an alarm for an incident is given if a threshold was reached.

In subsequent work, Abuelela et al. [106] introduced a probabilistic technique that uses vehicle to infrastructure communications to detect both temporary incidents, such as accidents and broken cars, and permanent road anomalies, including potholes, surface water, and speed bumps. The technique that they have developed is meant to supplement existing AID mechanisms and techniques especially in non-dense traffic. Their basic idea is to start with a set of beliefs, $\operatorname{Pr}[I]$, described as the a priori probability (or belief) of an incident $I$ at a given position on the road. When cars report a number of lane changes or hitting potholes, evidences E0s, correlated in both time and position, they update the belief by using a Bayesian mechanism $[106,107]$. They compute the a posteriori probability of an incident at the given location $I$ as

$$
\operatorname{Bel}(I)=\frac{\operatorname{Pr}[I] \operatorname{Pr}[E \mid I]}{\operatorname{Pr}[E]}=\alpha \operatorname{Pr}[I] \operatorname{Pr}[E \mid I]
$$

where $\operatorname{Pr}[E \mid I]$ is the likelihood, $E$ represents any evidence such as changing lanes or 
passing over a road anomaly and $\alpha$ is computed by the law of total probability as

$$
\frac{1}{\operatorname{Pr}[I] \operatorname{Pr}[E \mid I]+\operatorname{Pr}[\bar{I}] \operatorname{Pr}[E \mid \bar{I}]}
$$

The general idea of the technique is to start with some beliefs about having incidents on the road. If there are some evidences about many lane changes, sudden declaration and/or hitting road anomalies that are correlated in time and position, then they update the beliefs, using Bayesian theory, about having a road anomaly, permanent or temporary, that might exist and caused these many correlated evidences [106, 107].

It is very natural that most cars should change lanes to avoid an accident at a moderate distance away from the accident while a few cars change lanes at a very large or at a very small distance away from the accident. Hence, the normal distribution for lane changes with respect to the accident location applies. Non-dense traffic is assumed where cars can easily change lanes. On the other hand, the normal distribution may not be appropriate in dense traffic where lane changes may take longer to perform. Assume that an incident has occurred at position $y$ on the road and let $X$ be the random variable that keeps track of the position at which cars change lanes. Since, as postulated, $X$ is normally distributed, they write

$$
f_{X}(x)=\frac{1}{\sqrt{2 \pi}} e^{\frac{-(x-p)^{2}}{2}}
$$

On the other hand, in general, drivers notice a pothole only at short distance and this may impair their ability to change lanes to avoid it. Thus, they define the probability of changing lane at position $\mathrm{x}$ because of a pothole that exists at position $y$ as

$$
f_{X}(y)=I(L) \cdot \frac{1}{\sqrt{2 \pi}} e^{\frac{-(p-y)^{2}}{2}},
$$

where $I(L)$ is an indicator function returning 1 if the lane change $L$ occurs and 0 otherwise thus capturing the fact that some drivers may not change lanes and hit the pothole or maneuver around it.

\subsection{SUMMARY}

In this Chapter, we started with a general background about VANET. Then, we explained in brief the vehicle detection technology. We showed a comparison of incident detection algorithms. We explained traffic monitoring in VANET and data dissemination. Finally, an example of a system for incident detection (NOTICE) was explained. 


\section{CHAPTER 3}

\section{FRIEND - PHYSICAL COMPONENTS AND COMMUNICATION}

\section{PRIMITIVES}

The main goal of this chapter is to introduce the physical components and the communication primitives that FRIEND employs to implement incident detection, information propagation and to build various types of applications. By integrating its physical resources and computing and communication capabilities, FRIEND is expected to make a significant and lasting contribution to understanding the issues involved in aggregating traffic flow data collected by the huge fleet of vehicles on our roadways and streets into a comprehensive, near real-time synopsis of traffic flow conditions.

It is worth emphasizing that one of the most appealing features of FRIEND is that it is adding new, enhanced functionality to already installed infrastructure. To the largest extent possible, these enhancements are obtained by bringing to bear existing technology, even if such technology is not usually applied to applications related to traffic event detection and dissemination. Perhaps the best example of such an enhancement are the ubiquitous lane delimiters (commonly known as cat's eyes) installed, at regular intervals, along roadways and multi-lane city streets. The added functionality of the cat's eyes used by FRIEND is derived from wireless communication capabilities and various types of micro-sensors embedded in these devices. Using fairly standard technology, the cat's eyes (that we refer to as "smart cat's eyes") form a wireless network with nearest-neighbor connectivity that enables them to disseminate information about vehicles to other cat's eyes along the road and to various Roadside Units (RSUs). We note that, just like the cat's eyes mentioned above, RSUs are already deployed, at regular intervals, on some of our highways. Thus, in this regard, FRIEND offers the opportunity of a natural, and cost-effective, extension of the existing infrastructure. The same holds of the myriad on-board devices, mostly sensors and actuators, that vehicle manufacturers will soon start offering in order to ensure that their products are competitive, catering to a large array of consumer wants and needs.

Our discussion begins with a detailed survey of the physical infrastructure of FRIEND. We then go on to introduce the communication primitives used in FRIEND. Finally, we present our taxonomy of applications of FRIEND. 


\subsection{FRIEND - THE PHYSICAL COMPONENTS}

\subsubsection{SMART CAT'S EYES}

The intention is for smart cat's eyes (SCEs) to replace, in the near future, the ubiquitous cat's eyes, or lane separators that line our roadways and municipal corridors. We expect the SCEs to be the same size as regular cat's eyes, to be inexpensive (given a massive deployment), and to be robust and easy to maintain. We discuss the types of sensors, microprocessor, power consumption (battery), and amount of data that can be stored in the SCEs.

It is important to mention that for a decade, if not more, researchers in various countries have proposed all sorts of enhancements to the ubiquitous cat's eyes. For example, researchers in Scotland have introduced the concept of intelligent road studs [108]. One added functionality of the road studs is that they flash at night to indicate hazardous road conditions. They also have focused on improving the power consumption of road-studs [108]. However, to date, no wireless communication has been proposed for cat's eyes. Karpinski et al. [109] proposed the idea of augmenting regular cat's eyes with a processing and sensing capability. However, they just mentioned the applications that can be built using the enhanced cat's eyes without giving any details. To the best of our knowledge, we are the first to define SCEs in detail [110].

We now discuss in detail the physical components of our SCEs.

- Architecture: Each SCE is a compact, self-contained package. It contains several types of sensors (including magnetometers), a radio transmitter, an RFID tag, a micro-controller, a solar panel and a lithium battery;

- Power consumption: The SCE's main source of power is a rechargeable lithium battery. Under present-day technology it is also feasible to use non-rechargeable batteries. For example, the Tadiran TL-4935 lithium battery can supply $20 \mathrm{~mA}$ at $3.6 \mathrm{~V}$ for 90,000 hours, which is more than 10 years $[111,112]$. It has the same diameter as a $\mathrm{D}$ cell, but is $1 / 6$ the length. The battery is specified over most of the industrial temperature range, from $-30 \mathrm{C}$ to $+70 \mathrm{C}$. The battery voltage may vary between $3.5 \mathrm{~V}$ and $3.7 \mathrm{~V}$. Since the circuit is designed to operate at $3.0 \mathrm{~V}$ (to reduce the current drawn and therefore the power consumption), a $0.6 \mathrm{~V}$-drop silicon diode should be placed in series with the positive terminal of the battery. The resulting supply voltage range over temperature, $2.9 \mathrm{~V}$ to $3.1 \mathrm{~V}$, is within the specification range 
of all system components. However, we choose a rechargeable battery that has a solar panel as shown in Figure 6. The solar panel yields $0.2 \mathrm{~W} 2 \mathrm{~V} / 100 \mathrm{MA}$ with a battery pack Ni-MH1.2V/600MAH that has the following features [3]:

1. suitable for edge line of road and pavement;

2. after eight hours of normal sunlight or shining in the fog or rainy days for 120hs with different operating modes: sparking or steady shining, double-side or single-side;

3. can work more than three years, which promotes energy efficiency and environmental friendliness;

4. load-bearing: more than 20 tons as two reinforced veins are designed on the top edges to strengthen compression resistance and protect the solar panel against compression;

5. waterproof and unbreakable: the solar panel, electronics and optics are fitted inside.

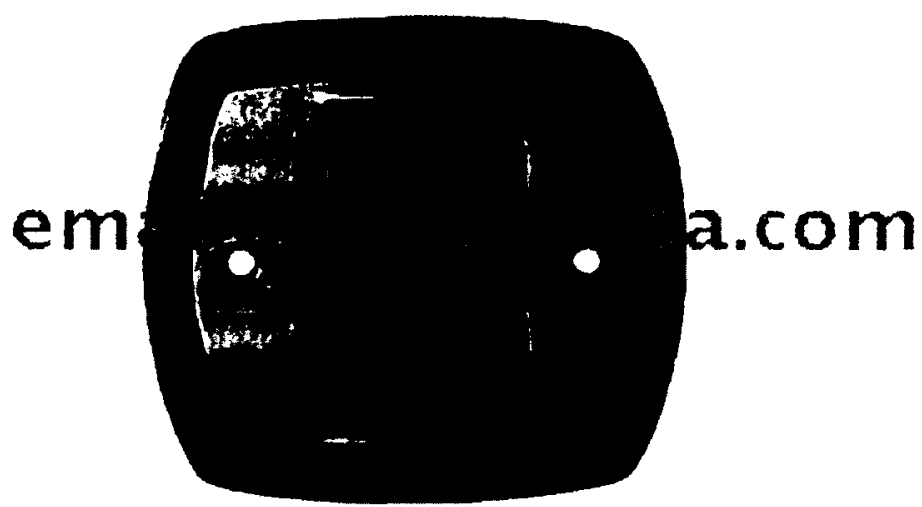

FIG. 6. Example of a SCE with solar panel: poly-crystalline silicon rechargeable Ni-MH batteries; http://www.alibaba.com [3]

- Communication technology: We assume that each SCE features a narrowband frequency-shift keying (FSK) data transceiver as well as one of many possible types of RFID tags compatible with the RFID readers installed in the smart wheels of vehicles, as will be discussed later in Section 3.2. 


\subsubsection{THE ROADSIDE UNITS (RSU)}

The second main component of FRIEND are the roadside units (RSUs) deployed at regular intervals (e.g. every kilometer or so), as illustrated in Figure 7. The RSUs placed on opposite sides of the roadway (e.g. RSUs $A$ and $A^{\prime}$ ) constitute a logical unit and are connected securely by optical fiber under the median. Unlike ILDs, adjacent RSUs along the roadway are not connected with each other, thus avoiding the huge cost of optical fiber. The RSUs are designed to be energetically self-sufficient, to have minimal needs for maintenance and service, and to be less expensive to build and maintain than ILDs. The role of the RSUs is to collect and aggregate traffic-related information from the passing cars as well as to exchange information, on an intermittent basis, with adjacent RSUs. When the RSUs become aware of an imminent or existing traffic incident, they disseminate this information to the traveling public.

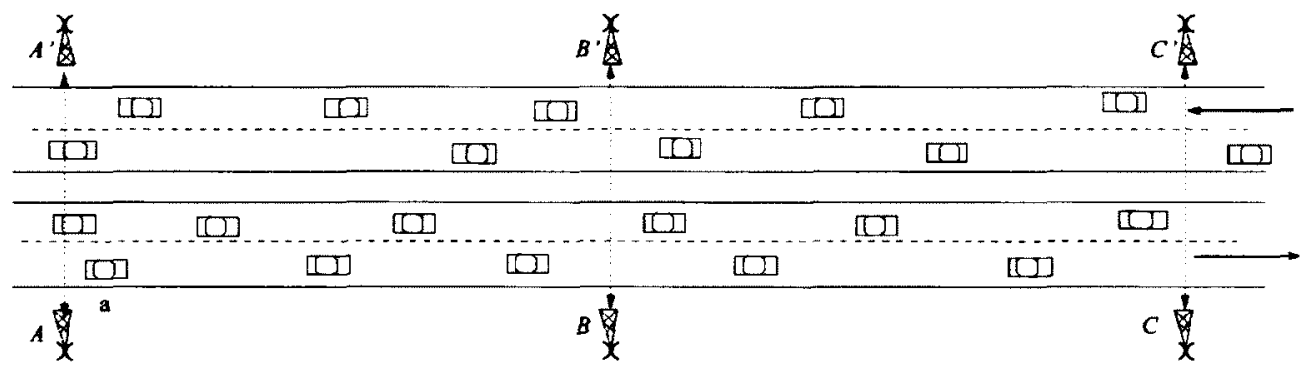

FIG. 7. A highway segment with several RSUs.

Each RSU contains a GPS, a radio transceiver, a laptop-class embedded computing device, such as the versatile low-power $\mathrm{PC} / 104$ system [113], powered by on-board battery packs charged by solar panels $[114,115]$. To minimize energy consumption, the RSUs are in a vigilant low-power mode when no cars are present and no traffic information needs processing. In this mode, we suggest two scenarios: first, the RSU will transmit only a periodic identification (every $\Delta t$ ) beacon to alert approaching cars; second, the RSU is operating at low power and is woken up by approaching vehicles. Assuming a RSU power consumption between $15 \mathrm{~W}$ and $20 \mathrm{~W}$ or, roughly, $480 \mathrm{Wh}$ of energy per 24 hours, this power could be supplied by a 12 V/200 Ah battery (e.g. a car battery) holding a total of 2,400 Wh of energy, which would discharge about $20 \%$. This can be fully recharged by a single $0.9 \mathrm{~m}^{2} 100 \mathrm{~W}$ solar panel (or two $50 \mathrm{~W}$ panels) with as little as four hours of sunlight 
per day $[116,114,115]$. Furthermore, assuming that the battery supports approximately 2,000 charging cycles during its lifetime, the $20 \%$ per day discharge rate of the battery translates into a $6+$ years of battery life $[117,116]$. We note that this is an estimate based on current battery and solar panel technologies.

Some versions of RSUs already exist on some highways as shown in Figure 8. Researchers anticipate using RSUs to improve connectivity on highways [118] and have studied their the optimal placement along highways [119]. However, it is required to standardize the components of the RSUs to build various types of applications.
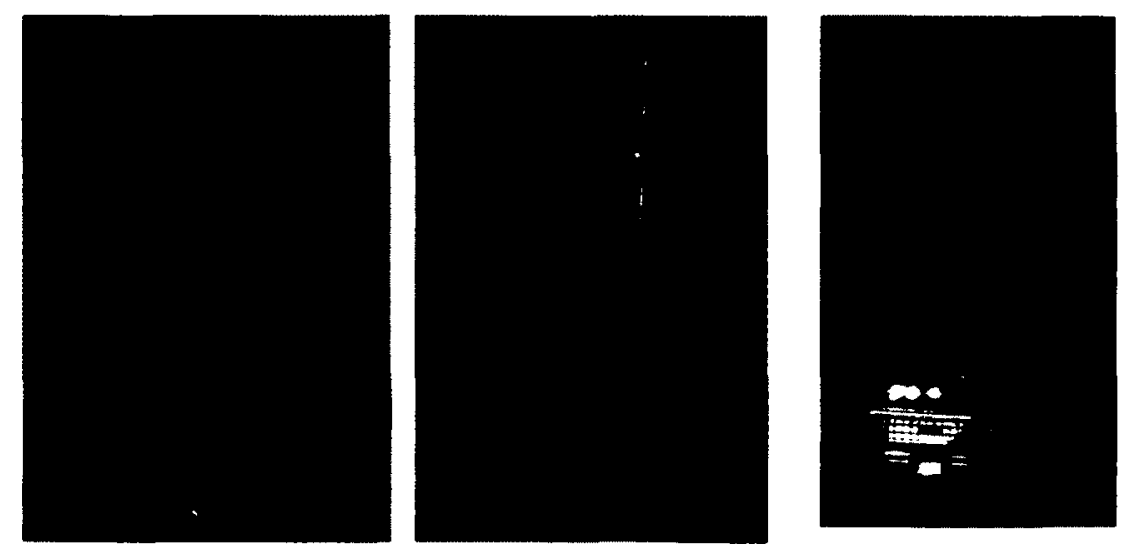

FIG. 8. Roadside units on Interstate 64, Virginia, USA.

\subsubsection{THE VEHICULAR MODEL}

Recent statistics show that in 2008 there were over 238 million passenger cars and trucks in the US, a vehicular fleet that increases yearly by almost seven million new cars [120]. In an effort to help their vehicles compete in the marketplace, car and truck manufacturers are offering more and more potent on-board devices, including powerful computers, a large array of sensors, on-board radar devices, cameras, and wireless transceivers. These devices cater to a set of customers that expect their vehicles to provide a seamless extension of their home environment populated by sophisticated entertainment centers, access to Internet and other similar wants and needs [121, 122]. 


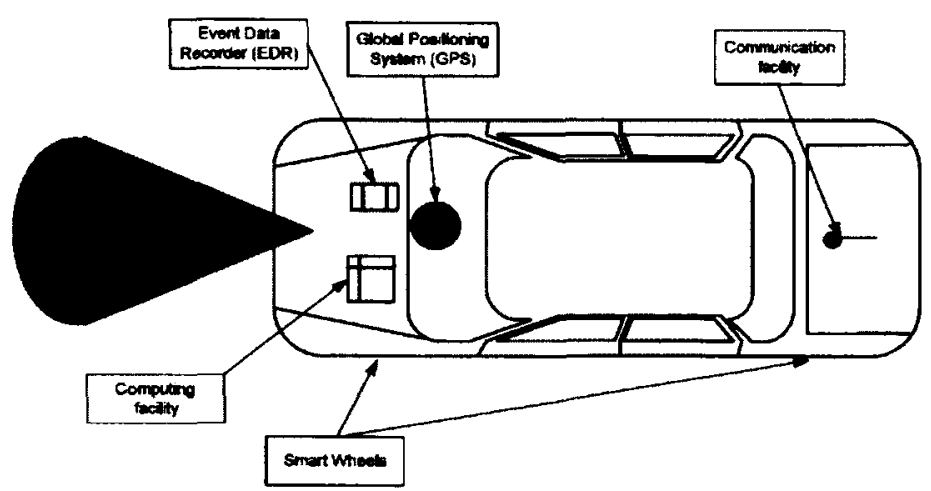

FIG. 9. Several on-board sensors in present-day vehicles.

In the literature, the term smart vehicles refers to certain sophistication of on-board equipment enabling vehicles to perform computing operations, carry out wireless communications, and utilize sensor data capturing the operational status of mechanical parts. In this thesis, we assume vehicles to be equipped with the following on-board sensors/devices as shown in Figure 9:

- Event Data Recorder(EDR): According to the National Highway Traffic Safety Administration (NHTSA) [123], "an EDR is a device or function in a car that records the car dynamic, time-series data, etc". In its 2006 ruling [124], NHTSA has mandated that starting September 2010 an EDR will have to be installed in vehicles with an unloaded weight of less than 5,000 lbs. NHTSA has since updated its ruling to give vehicle manufacturers until September 2012 to be in compliance with the original ruling. The EDR is responsible for recording mobility attributes including acceleration, deceleration, lane changes and the like. Each such transaction is associated with an instantaneous GPS reading. All of the car's sub-assemblies, including the speedometer, engine temperature, oil pressure sensors, tire pressure sensors, and sensors for outside temperature and road condition feed their readings into the EDR $[125,126]$. While many cars are already equipped with EDRs, it is less well known that some car manufacturers have been offering EDR devices on a voluntary basis. For example, some GM and Toyota cars as old as model year 1994 were equipped with an EDR-like device able to store retrievable data. The EDR will be described in detail in Subsection 3.1.4;

- GPS receiver: The GPS receiver provides an accurate location of the vehicle. The 
location information includes latitude, longitude, altitude, speed and heading information. We convert the location given by the GPS device reading which is known by (Latitude and Longitude) $\left(x_{i}, y_{i}\right)$ into $\left(l_{i}, o_{i}\right)$ where $l_{i}$ represent the lane and $o_{i}$ represent the offset from the previous RSU [127], as shown in Section 3.1.5;

- Wireless transceiver: The transceiver uses the standard Dedicated Short Range Communications (DSRC) [128] specially designed for automotive use and a corresponding set of protocols and standards (IEEE 802.11p [11, 12]). The transmission range of DSRC is 300 meters (for the new DSRC the transmission range is about 1000 meters). DSRC for intelligent transportation systems operates in the $5.9 \mathrm{GHz}$ and (U.S.) or $5.8 \mathrm{GHz}$ band (Japan, Europe);

- Digital map: The digital map is an electronic map that has the locations of all RSUs locations on the highways;

- Smart wheels: We assume that vehicles' wheels have a wireless communication capabilities allowing them to send instantaneous speed and traction (or loss thereof) information to SCEs. The smart wheels contain an RFID reader of a type compatible with the RFID tags in the SCEs;

- Temperature sensor: We assume that vehicles can measure the temperature of the highway using an on-board temperature sensor;

- Radar: We assume that vehicles come complete with an on-board radar device that can measure the headway distance as explained in Chapter 4. This technology already exists. For example, Delphi Electronically Scanning Radar (ESR) [129] is an example of a radar with medium range of $60 \mathrm{~m}$ and long range up to $174 \mathrm{~m}$ as shown in Figure 10.

- Electronic stability control system: In 2004, the NHTSA created its Light Vehicle Handling and Electronic Stability Control (ESC) program. When first conceived, this program emphasized the development of test maneuvers and analysis methods capable of objectively quantifying handling. The EDR records the number of occurrences of the electronic stability control system signaling a loss of steering. In FRIEND, num ${ }_{E S C}$ is the number of losses that occurred in the last segment. In 


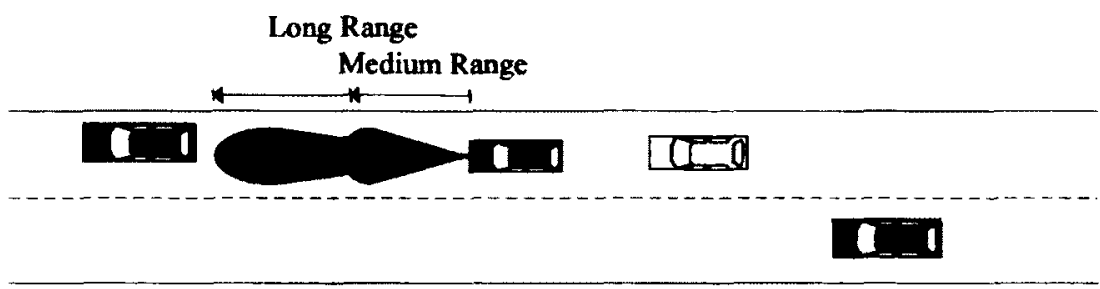

FIG. 10. An example of on-board radar installed in a vehicle.

Figure 11, we add to the ESC block diagram the wireless notification system that communicates not only with the vehicle itself but also with both surrounding vehicles and the infrastructure nodes (SCEs). Driver input may consist of the steering angle, accelerator position and brake pressure [130].

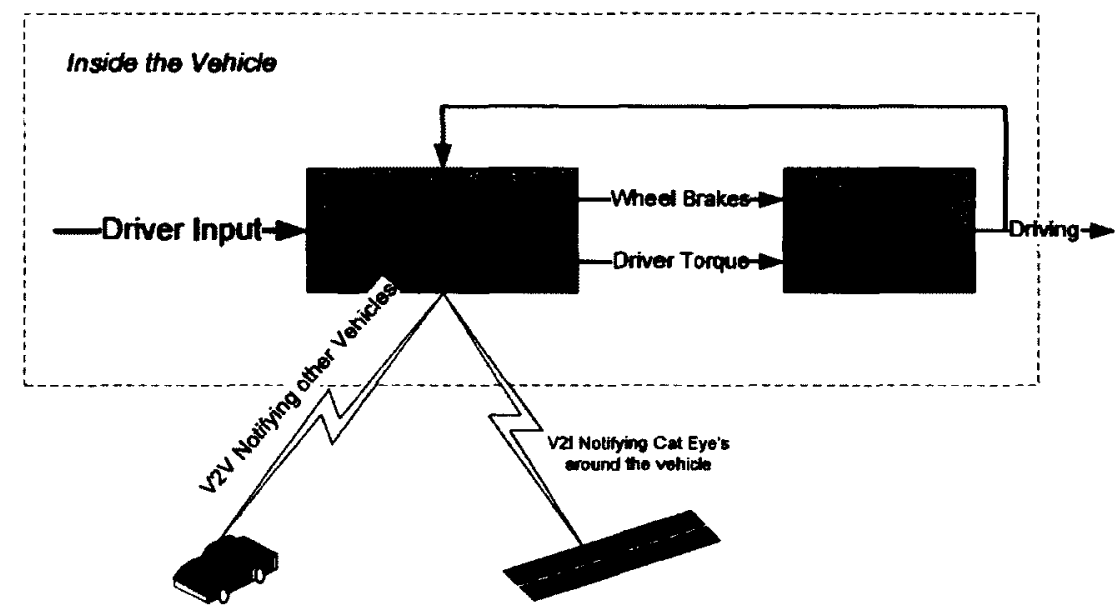

FIG. 11. Electronic stability control block diagram.

\subsubsection{THE EVENT DATA RECORDER}

In this subsection, we discuss the details of the Event Data Recorder installed in vehicles. The EDR is responsible for recording mobility attributes including acceleration, deceleration, lane changes, etc. Each such transaction is associated with an instantaneous GPS reading. All of the car's sub-assemblies, including the speedometer, engine temperature, oil pressure sensors, tire pressure sensors, and sensors for outside temperature and 
road condition feed their readings into the EDR [125, 126].

As already mentioned, in this thesis we assume that vehicles are equipped with an EDR which is a tamper-proof device that keeps track of the data recorded by vehicle's sensors. Our assumption extends the normal responsibilities of the EDR (as described by the NHTSA ruling [124]) from a data recorder to a monitoring device that records all the attributes describing the mobility of vehicles such as velocity, lane changes, among many others.

EDR designs have been developed independently by each car and truck manufacturer to meet their own vehicle-specific needs. There is no common format or protocol for data collection, retrieval, or maintenance. Currently, several organizations are actively developing standards for vehicle EDRs used in commercial and passenger vehicles. These groups are defining industry standards or recommended practices for EDR formatting, methods of retrieval, and procedures for record archiving. However, current research focuses on data retrieval and storage for vehicle crashes or accidents [131].

The design of FRIEND prescribes, in minute detail, the type of data exchanges that take place between the EDR and RSUs. Moreover, we calculate the required size of the beacon to be transferred from the vehicle's EDR to RSU. Recall that, as mentioned above, vehicles are assumed to have an on-board GPS receiver enabling the vehicle to track its location (geographic position), and on-board computing devices allowing the vehicle to perform simple calculations including encryption and other vehicles' position, and communication devices (DSRC compliant) to propagate/receive information.

\subsubsection{THE EDR DATA FORMAT}

In this subsection, we discuss the format of the data recorded by the EDR. We summarize these data formats as follows:

1. Location: We convert the location given by the GPS device, which is known as latitude and longitude $\left(x_{i}, y_{i}\right)$ into $\left(l_{i}, o_{i}\right)$ where $l_{i}$ represents the lane and $o_{i}$ represents the offset from the previous RSU [127], as shown in Figure 12.

$\because$ Number of possible lanes $=6$ lanes

$\therefore 3$ bits to represent 8 lanes

$\because$ Distance for offset $<4$ kilometers

$\therefore 11$ bits to represent offset 


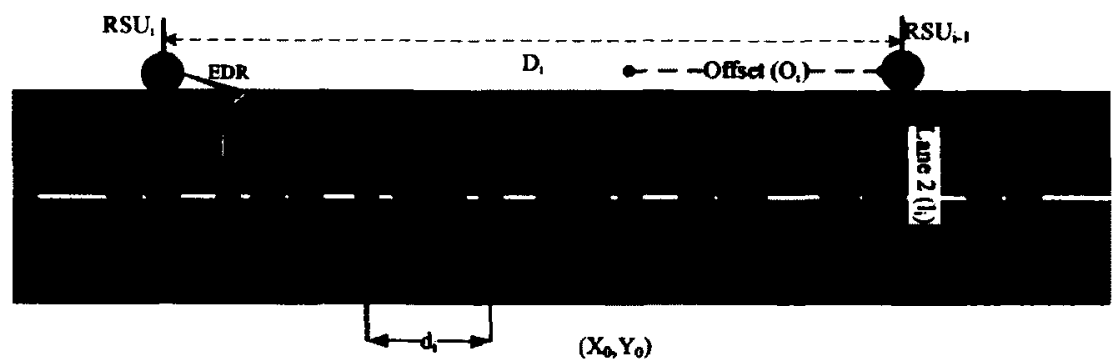

FIG. 12. Converting a GPS reading to the (Lane, Offset) form.

It follows that two bytes are sufficient to represent location. This method saves 10 bytes as compared to other techniques [132];

2. Speed: We keep track of the minimum and maximum speeds recorded in the previous segment (from last RSU).

- $\left(l o c_{i}, \min S_{i}\right)$ : location and minimum speed recorded in the last segment;

- $\left(l o c_{i}, \max S_{i}\right)$ : location and maximum speed recorded in the last segment;

- The average speed can be calculated from the time of data exchange with $R S U_{i}$ and $R S U_{i-1}$ using the formula $V_{\text {avg }}=\frac{D}{t_{i}-t_{i-1}}$, where $D$ is the distance between two adjacent RSUs, and $t_{i}, t_{i-1}$ are the times recorded when communicating with $R S U_{i}$ and $R S U_{i-1}$ respectively.

Number of bits required for speed:

- Solution 1: We present the speed from 0 to $180 \mathrm{Km} / \mathrm{hr}(50 \mathrm{~m} / \mathrm{sec})$ in 6 bits;

- Solution 2: We present the speed in 3 bits divided to 8 ranges: $[0-10] \mathrm{km} / \mathrm{hr},[10-25] \mathrm{km} / \mathrm{hr},[25-40] \mathrm{km} / \mathrm{hr},[40-60] \mathrm{km} / \mathrm{hr},[60-$ $80] \mathrm{km} / \mathrm{hr},[80-100] \mathrm{km} / \mathrm{hr},[100-120] \mathrm{km} / \mathrm{hr}$, and more than $120 \mathrm{~km} / \mathrm{hr}$ as shown in Figure 13;

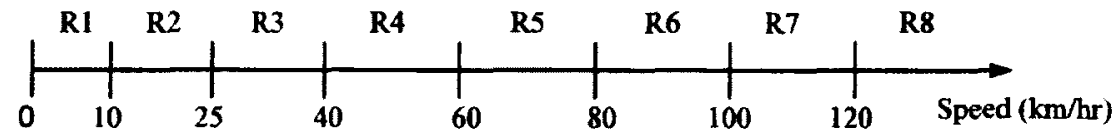

FIG. 13. Speed partitioned into ranges - saved in EDR 
3. Steering system: The EDR records lane changes using a combination of steering system feedback and GPS readings. The EDR records location and lane change direction ( $l o c_{i} 2$ bytes, direction 2 bits). Direction can take the values of (left, right, null) where null represents the situation where the driver starts to change the lane but returns to the same lane.

\subsection{DATA COMMUNICATION IN FRIEND}

In this section, data exchange and communication between the various entities that make up FRIEND are discussed. We begin with a quick summary of the FCC-mandated Dedicated Short Range Communications (DSRC) for fast communications specially dedicated for automotive use [128]. This is then continued with a description of the various types of communication that occur in FRIEND. In Figure 14, various types of communications are shown [133]. Vehicles using their smart wheels broadcast in a short range relevant information to the intention of SCEs, vehicles communicate and exchange information with RSUs, SCEs may communicate with neighboring SCEs on demand, RSUs can demand information from SCEs, and finally RSU to RSU communication occurs when information is requested or propagated.

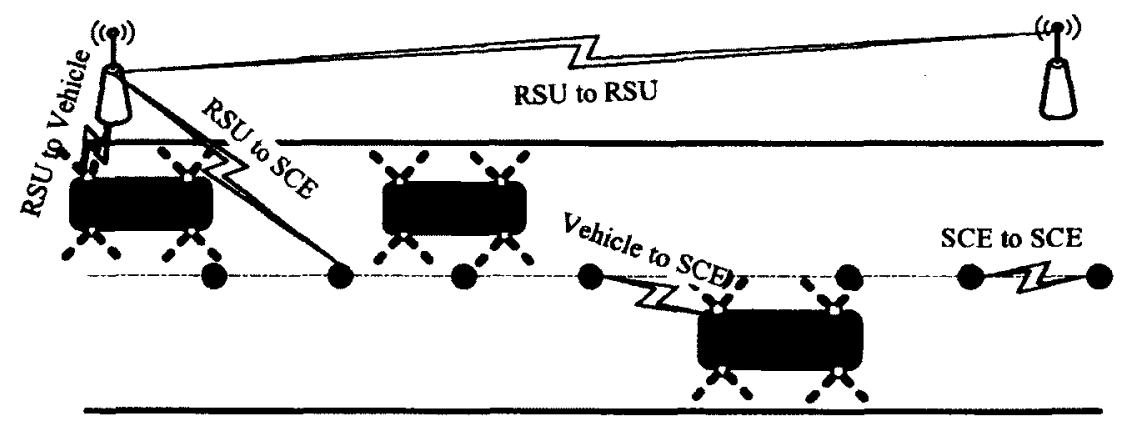

FIG. 14. Illustrating communications between various entities in FRIEND.

\subsubsection{DEDICATED SHORT RANGE COMMUNICATIONS}

DSRC is a suite of standards at the heart of the communication of vehicular messages. The fast exchanging of messages, combined with knowledge about other moving vehicles 
that may not be visible to drivers in a timely manner extend the safety concepts beyond the dreams of most of the public [134]. Wireless Access in Vehicular Environment (WAVE) is a term used to describe the suite of IEEE P1609.x standards that are focused on MAC and network layers. WAVE is fairly complex and is built over the IEEE 802.11 (WiFi) standards by adapting the protocols to guarantee fast reliable exchange of safety messages. WAVE is the core part of DSRC; however, either of the two terms is commonly used arbitrarily. In some cases, the term DSRC is used as a more general term compared to WAVE [128, 134].

The DSRC community attempted to standardize the $915 \mathrm{MHz}$ using the ASTM framework but quickly thought of the IEEE 802.11 approach and the $5.9 \mathrm{GHz}$ as a direct way to benefit from its ad-hoc mode. The ad-hoc mode of IEEE 802.11 resembles the situation of vehicle-to-vehicle communications and hence, simplifies the development of DSRC [128, 134]. Almost a decade of DSRC standards development has resulted in the IEEE 802.11p standards along with IEEE 1609.x, both standards represent together proposed DSRC suite of standards. DSRC is currently considered the most promising wireless standard that can be used to connect infrastructure (like roadside) to vehicle (I2V) and vehicle-to-vehicle (V2V). The DSRC standard is based on the WiFi architecture. Relevant application like Vehicle-Infrastructure Integration (VII now called Connected Vehicles), Cooperative Intersection Collision Avoidance Systems (CICAS) and others have developed their architecture with DSRC services in mind [128, 134].

Table 4 illustrates the various types of communications that occur in FRIEND. In the following subsections, we discuss each of these in detail.

\subsubsection{VEHICLE TO SCE COMMUNICATION}

The first type of communication is RFID-based and takes place between the smart wheels of vehicles and SCEs. The RFID reader in the smart wheels allows the vehicle to inform the SCE about speed, stability loss due to road conditions (if any), and ambient temperature. The SCEs collect data sent from vehicles every $\Delta t$, where $t$ depends on highway conditions. The RFID reader in the smart wheels transmits an object identity using electromagnetic waves. In the SCE, an RFID tag stores its ID in memory. The RFID reader which is installed in the vehicle wheels emits $R F$ radio waves eliciting a signal back from the tag. We use RFID with radio range (up to approximately $3 \mathrm{~m}$ ). The most important 
TABLE 4. Different types of communications in FRIEND.

\begin{tabular}{|c||c|}
\hline Communication between entities & Communication type \\
\hline \hline Vehicle to SCE & RFID \\
\hline Vehicle to RSU & DSRC \\
\hline SCE to SCE & Zigbee \\
\hline SCE to RSU & narrowband FSK data transceivers \\
\hline RSU to SCE & Broadcast (WiFi) \\
\hline RSU to RSU & Radio \\
\hline EDR to RSU & Emergency DSRC \\
\hline
\end{tabular}

benefit of an RFID tag is the battery-free operation. A tag works without a power source since it gathers energy from a reader's waves [135].

RFID is indeed used in various vehicular applications. For instance, in the Automatic Toll Collection (ATC) system, roadside RFID readers identify passing vehicles by reading tags on them and then automatically charge the fare. Other researchers have used RFIDbased vehicular communications $[135,136,137]$.

The two RFID readers inside the vehicle are placed as shown in Figure 15 with the front reader mounted close to the front axle of the vehicle, and the rear one installed at the rear of the vehicle (in the middle of the wheels). The front RFID reader $\sharp 1$ will attempt to establish communication with the tag in the SCE that the vehicles passes. If communication is not successful another attempt is made using the second RFID reader 2. Table 5 shows the difference between passive and active tags [137].

TABLE 5. Illustrating the difference between active and passive tags.

\begin{tabular}{|c||c||c|}
\hline Comparison & Active tags & Passive tags \\
\hline Transmission range & $>10 \mathrm{~m}$ & $<10 \mathrm{~m}$ \\
\hline Battery life & around 1 year & no battery \\
\hline Security & Strong & Weak \\
\hline
\end{tabular}

\subsubsection{SCE TO SCE COMMUNICATION}




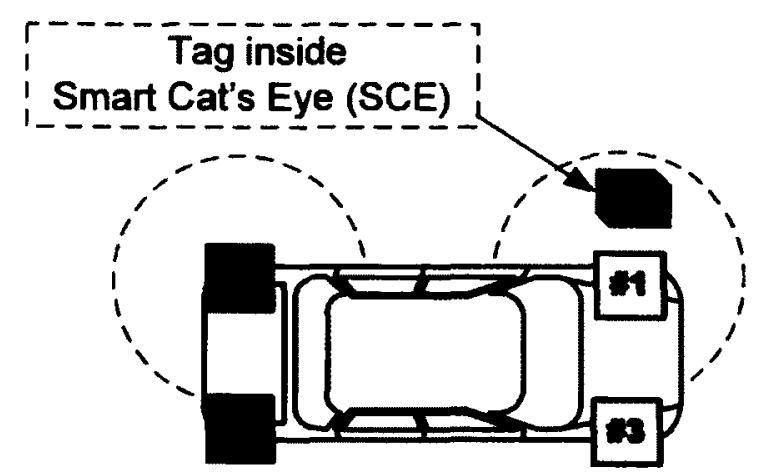

FIG. 15. RFID readers in vehicles.

The second type of communication is between neighboring SCEs along the roadway. In case of an incident, as we will see in Chapter 6, the RSUs can request information from SCEs. We use Zigbee to communicate between SCEs. Zigbee is based on IEEE 802.15.4 and is a suite of high-level communication protocols using small, low-power digital radios. Zigbee can transfer $250 \mathrm{Kbps}$ with a connection setup time around $30 \mathrm{msec}$. The range of communication in Zigbee is from 10 to 100 meters. Table 6 shows a comparison between different wireless technologies [138].

TABLE 6. A comparison between short-range wireless technologies.

\begin{tabular}{|c|c|c|c|c|}
\hline Specifications & $\begin{array}{l}\text { Blue- } \\
\text { tooth }\end{array}$ & ZigBee & WiFi & RFID \\
\hline IEEE & 802.15 .1 & 802.15 .4 & $\begin{array}{l}802.11 \\
\mathrm{a} / \mathrm{b} / \mathrm{g}\end{array}$ & - \\
\hline Frequency & $2.4 \mathrm{GHz}$ & $\begin{array}{l}868 / 915 \\
\mathrm{MHz}\end{array}$ & $\begin{array}{l}2.4 \mathrm{GHz} \\
5 \mathrm{GHz}\end{array}$ & $\begin{array}{l}\text { LF, HF, } \\
\text { UHF, } \\
\text { Microwave }\end{array}$ \\
\hline Data rate & $1 \mathrm{Mb} / \mathrm{s}$ & $250 \mathrm{Kbps}$ & $54 \mathrm{Mb} / \mathrm{s}$ & $1-200 \mathrm{~Kb} / \mathrm{s}$ \\
\hline $\begin{array}{l}\text { Connection } \\
\text { setup time }\end{array}$ & $\geq 3 \mathrm{sec}$ & $\sim 30 \mathrm{msec}$ & N/A & $\begin{array}{l}\text { depend on } \\
\text { RFID type }\end{array}$ \\
\hline Max range & $10 \mathrm{~m}$ & $10-100 \mathrm{~m}$ & $100 \mathrm{~m}$ & $0.01-100 \mathrm{~m}$ \\
\hline
\end{tabular}

\subsubsection{SCE TO RSU COMMUNICATION}


Spread-spectrum radio communications are very appealing to practitioners because they are immune to jamming and adjacent channel interference, and can be legally operated at higher data rates than narrowband, systems [111]. However, spread-spectrum systems need to synchronize to communicate. This typically requires a long time interval (several seconds) where the transmitter is drawing power but not transmitting bits. A typical ISM band spread-spectrum transmitter might consume $500 \mathrm{~mA}$ for several seconds before becoming available to transmit bits.

In FRIEND, it is essential to save power in SCEs and, therefore, spread-spectrum communication is not an option. For example, simple narrowband FSK radio data transmitters turn on within milliseconds and draw only $10-20 \mathrm{~mA}$. Adjacent-channel interference and jamming are very real problems, but can be mitigated by using a frequency-agile narrowband system. Since this communication does not require a high data rate, we choose to use narrowband FSK data transceivers in SCEs, as in [111].

\subsubsection{VEHICLE TO RSU COMMUNICATION}

Vehicles can wake up the RSU in case of sparse traffic using a low power beacon that informs the RSU of traffic activity. The moment a vehicle enters the range of a RSU (observe that vehicles can identify the area by using the built-in digital map), it starts exchanging data with the RSU. The EDR data exchanged between the vehicle and the RSU, subject to the conditions stipulated in the next paragraph, includes

- Time of exchanging data with previous RSU;

- Minimum speed recorded in the last segment;

- Lane changes recorded in the last segment;

- Number of electronic stability loss signals recorded in the last segment.

A car approaching a RSU is either entitled to drop off EDR data with the RSU or else it is considered "new" and is not allowed to do so. Communication between vehicles and RSU is explained in details in Chapter 5.

\section{Reasoning about the RSU coverage area}

Successful data collection/information exchange between a passing car and the RSU depends on the amount of time the car is within the coverage area (radio range) of the RSU. 
To simplify matters assume that that cars travel at constant speed $s$. Let the random variable $X$, with distribution function $F_{X}$, keep track of the time between the moment a car enters the RSU coverage area and the time it received the first beacon; let the random variable $Y$, with distribution function $F_{Y}$ denote the time it takes to perform the information exchange. Referring to Figure 16, it is clear that successful data exchange hinges on the convolution $X+Y$. Let, $z \geq 0$ be arbitrary and let $A$ be the planar domain defined as $A=\{(x, y) \mid x, y \geq 0 ; x+y \leq z\}$. With $F_{X, Y}$ denoting the joint distribution function of $X$ and $Y$ we write

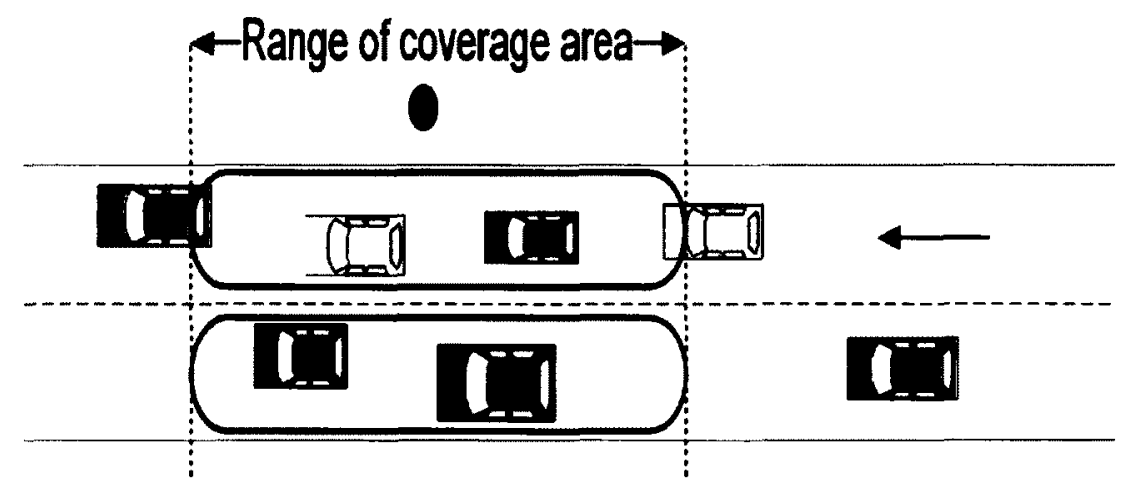

FIG. 16. Illustrating the coverage area of a RSU.

$$
\begin{aligned}
\operatorname{Pr}[\{X+Y \leq z\}] & =\iint_{A} \mathrm{~d} F_{X, Y}(x, y)=\iint_{A} \mathrm{~d} F_{X}(x) \mathrm{d} F_{Y}(y)(y) \\
& =\int_{0}^{\infty} \mathrm{d} F_{X}(x) \int_{z-x}^{\infty} \mathrm{d} F_{Y}(y)(y)=\int_{0}^{\infty}\left[1-F_{Y}(z-x)\right] \mathrm{d} F_{X}(x \zeta)
\end{aligned}
$$

Now, assuming that the desired coverage area has size $D$, that $X$ is uniformly distributed in $\left[0, \frac{D}{s}\right]$ and that $Y$ is general, we have

$$
\operatorname{Pr}\left[\left\{X+Y \leq \frac{D}{s}\right\}\right]=\frac{s}{D} \int_{0}^{\frac{D}{s}}\left[1-F_{Y}\left(\frac{D}{s}-x\right)\right] \mathrm{d} x
$$

We have experimented with the following estimates for the entries in Figure 16:

- beacon intervals $T_{b}$ starting from $100 \mathrm{~ms}$ (typical for 802.11-based wireless systems) to around $1.1 \mathrm{~s}$; 
- 50 ms for encrypting and decrypting a 1 kbyte "HELLO" message;

- $8 \mathrm{~kb}$ maximum data transferred between car and RSU at data rates of 250,500 , and $1,000 \mathrm{kbps}$;

- $300 \mathrm{~ms}$ for encrypting and decrypting data transferred between car and RSU;

\subsubsection{ROLE-BASED VEHICLE TO RSU COMMUNICATION}

There are exceptional cases where the communication between RSUs and passing cars needs to be augmented to allow authorized vehicles to interact with FRIEND in a predetermined, role-based, fashion. This feature is essential to the interaction with first responders, ambulances, fire fighters, local police, and traffic management personnel in case of emergency operations. In such scenarios, authorized vehicles using a special encryption key will be allowed to load essential information onto individual RSUs. For example, police cars may load information related to planned lane closures, suggested detour routes, as well as the availability of resources in the case of a planned evacuation.

\subsubsection{RSU TO RSU COMMUNICATION}

There are cases where neighboring RSUs along the roadway need to communicate directly, as opposed to communicating by "courier" having vehicles carry messages between them.

For example, a RSU can request information or inform a neighbor RSU in case of accident or sudden change in traffic flow. Under normal traffic conditions, adjacent RSUs along the roadway do not communicate with each other directly, relying instead on passing vehicles to carry information between them. However, whenever time-critical messages need to be exchanged, adjacent RSUs can, and do, communicate directly for short periods of time using a suitable radio interface.

In order to make the communication between adjacent RSUs secure, each adjacent pair, say $A$ and $B$, of RSUs along the roadway (see Figure 7) shares a time-varying symmetric key $\mu(A, B, t)$ used to encrypt, at time $t$, the data exchanged between them. Since the RSUs are synchronous (by virtue of the GPS), they switch from one key to the next in a pre-established order based on their local time.

Referring to Figure 7, assume that RSU $A$ has a non time-critical message $m$ for RSU $B$. $A$ will encrypt $m$ with $\mu(A, B, t)$ and will upload it onto passing car $a$. When car $a$ 
reaches $B$, the message $m$ will be dropped off by car $a$ and decoded by $B$. In turn, $B$ may decide to send a message to RSU $C$. This would be done using the symmetric key $\mu(B, C, t)$, known only to $B$ and $C$. It is important to note that, given a sufficiently large set of keys in the key-chain, the RSU to RSU encryption keys appear random to an external observer. A key component of FRIEND (that, however, will not be addressed in this thesis) is to identify robust schemes that allow adjacent RSUs to communicate securely.

In order to communicate with passing cars, the RSU radios use multiple access technology to ensure independent coverage of each traffic lane as illustrated in Figure 16. Each lane in the RSU coverage area is assigned non-overlapping sets of orthogonal channels to ensure that there is no interference among the various links established when cars in adjacent lanes communicate with the RSU simultaneously. Furthermore, in case several vehicles are in the same lane within the coverage area of the RSU, the use of multiple access schemes enables the RSU to communicate with all of them.

While the best choice of the length, $D$, of the RSU's coverage area is yet to be determined, preliminary estimates discussed in Subsection 3.2.5 indicate that for cars traveling at $70 \mathrm{mph}$ a value of $D=45 \mathrm{~m}$ suffices. Such a radio range may be covered by ZigBee, UWB or, indeed, any suitable short-range communication technology $[139,140,138,141]$.

\subsection{A TAXONOMY OF APPLICATIONS OF FRIEND}

The past decade has witnessed a growing interest in vehicular networking and its multiple applications. The initial vision that had fueled research in vehicular networking had originated in an altruistic impulse, namely that radio-equipped vehicles can somehow network together and, by exchanging and aggregating individual views, can keep the drivers informed about potential traffic safety risks and can heighten their awareness of road conditions and other traffic-related events. The unmistakable promise of vehicular networking has lead to a rapid converge with ITS leading to the emergence of Intelligent Vehicular Networks, expected to revolutionize the way we drive by creating a safe, secure, and robust environment that will eventually pervade our highways and city streets. As already mentioned, in support of vehicular communications, the US Federal Communications Commission (FCC) has allocated $75 \mathrm{MHz}$ of spectrum in the 5.850 to $5.925 \mathrm{GHz}$ band specially allocated for DSRC.

There are many possible classifications for vehicular networks applications [142]. The most basic one is to partition these applications intro three groups: safety, comfort and entertainment [143, 144]. Drivers may accept a delay or even a loss of data in commercial 
applications. On the other hand, drivers will not accept the chance of risking their life or even relying on safety applications that have a small margin of error. Various types of applications have been discussed recently by researchers. Emergency Electronic Brake Light is the first vehicle to vehicle cooperative active safety application that establishes EEBL messages. Cooperative Intersection Collision Avoidance Systems is another well known example which tries to alert drivers of intersection collisions. Other applications include parking location assistance; food drive-through payment; road toll payment; download movies, games, and $\mathrm{mp} 3$; and remote diagnostics for vehicle problems introduced in recent research papers.

Real-time reliable communications are a great challenge to researchers and vehicle developers. A specific channel in DSRC is dedicated solely to safety communication. Other channels are reserved for accident avoidance applications and high power public safety communication usages. It was recently noticed that the DSRC spectrum set aside by the FCC by far exceeds the needs of traffic-related safety applications. This observation has motivated the emergence of a host of other applications that can take advantage of the allocated spectrum. Not surprisingly, we see more and more third-party providers offering non-safety-related applications ranging from location-specific services, to on-theroad peer-to-peer communications, to Internet access, to on-line gaming and other forms of mobile entertainment. In due time, we will see the emergence of commercial applications targeted at the traveling public and distributed via the excess bandwidth in DSRC. As a pleasant side benefit, the unsightly billboards that flank American highways will disappear and will be replaced by in-vehicle advertising that the driver can filter according to their wants and needs.

In FRIEND, we classify VANET applications vertically, into several distinct levels, depending on the required data, type of network communications and real-time processing needs. Figure 17 captures our taxonomy of applications.

We define the VANET applications levels as follows:

Level 0 applications: These are applications that do not require communication. A group of sensors can be used to build this type of application;

Level 1 applications: These are applications that involve communication with a RSU. However, processing can be done off-line or data analysis can be performed over a 


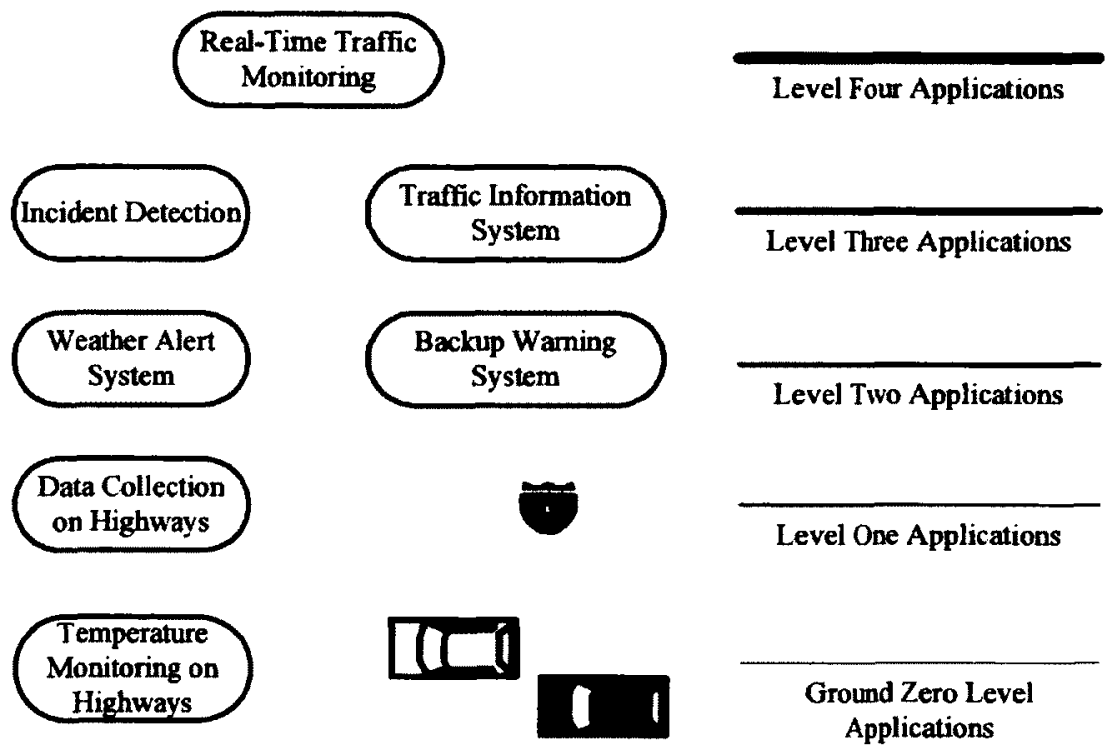

FIG. 17. A taxonomy of vehicular networks applications.

long period of time;

Level 2 applications: Like Level 1 applications, these applications involve communication. However, in this case the analysis of data must be performed faster as a message may be broadcast to vehicles as a warning message;

Level 3 applications: These applications are Level 2 applications that require an update of the status of an event over time;

Level 4 applications: These are the most demanding applications, requiring real-time processing and real-time update of status of an event or traffic.

In Table 7, we describe each level and give an example of the application.

\subsection{SUMMARY}

In this chapter, we introduced the main physical components and the communication capability of FRIEND. First, we explained the new SCE nodes that would replace the old cat's eyes on highways. We explained the components, features, and communication 
TABLE 7. Example of vehicular networking applications.

\begin{tabular}{|l||l|l|}
\hline Application level & Description & $\begin{array}{l}\text { Example of an appli- } \\
\text { cation }\end{array}$ \\
\hline Level 0 & $\begin{array}{l}\text { local sensors in the vehi- } \\
\text { cle, no communication is } \\
\text { involved }\end{array}$ & $\begin{array}{l}\text { measuring the highway } \\
\text { temperature }\end{array}$ \\
\hline Level 1 & $\begin{array}{l}\text { requires a communication } \\
\text { with a management center } \\
\text { or road side unit }\end{array}$ & $\begin{array}{l}\text { data collecting on a } \\
\text { highway }\end{array}$ \\
\hline Level 2 & $\begin{array}{l}\text { an analysis and broadcast- } \\
\text { ing of a message or warn- } \\
\text { ing to other vehicles }\end{array}$ & weather alert systems \\
\hline Level 3 & $\begin{array}{l}\text { requires a feedback or up- } \\
\text { dates of the status }\end{array}$ & incident detection \\
\hline Level 4 & $\begin{array}{l}\text { real-time processing and } \\
\text { feedback }\end{array}$ & $\begin{array}{l}\text { real-time monitoring of } \\
\text { traffic flow }\end{array}$ \\
\hline
\end{tabular}

technology. Second, we showed the RSU nodes, which are a modified version of the current RSUs already existing on some highways in US. We showed the expected vehicular model with a complete discussion of the EDR installed in vehicles and its role.

Then, we described the communication between the various entities in FRIEND, which will be explained in detail in Chapter 5. Finally, we showed our classification of VANET applications into several distinct levels depending on the required data, type of communication and processing. 


\section{CHAPTER 4}

\section{REASONING ABOUT TRAFFIC FLOW PARAMETERS}

Recall that FRIEND relies on EDR data collected from passing cars. Naturally, the EDR data has to be related to the underlying traffic flow out of which it was sampled. Converting raw EDR data to various parameters of the traffic flow need is one of the main contributions of FRIEND.

This chapter has two related goals. First, it reviews a number of important traffic parameters that underlie the aggregation of traffic-related data collected by FRIEND. The details are presented in Sections 4.1, 4.2 and 4.3. Second, the chapter discusses the techniques that FRIEND uses to learn and reason about these parameters. The actions performed by the RSUs to make this conversion are discussed in Section 4.4.

\subsection{TRAFFIC FLOW PARAMETERS}

As mentioned in Chapter 3, the RSUs are collecting data from passing vehicles. The RSU's directional antenna can communicate with vehicles the moment they reach its communication area. The RSU collects two important parameters that helps in measuring the flow of the highway. The parameters are speed and headway distance as discussed in the following subsections. Also, the RSUs keep track of the cluster size per lane as an indication of the capacity of the highway. In the following sections, we briefly review a number of important traffic-related parameters including the headway distance, safe headway distance, clustering and the relationship between these variables and the traffic flow on the highway.

The goal of this section is to review a number of fundamental traffic parameters that will play a crucial role in FRIEND. The general traffic theory parameters include:

- Rate of flow (vehicles per unit time),

- Speed (distance per unit time),

- Travel time over a known length of road,

- Occupancy (percent of time a point on the road is occupied with vehicles, 
- Density (vehicles per unit distance),

- Time headway between vehicles (time per vehicle),

- Space headway between vehicles (distance per vehicle).

In order to use these parameters, FRIEND needs to measure them. Measuring these parameters can be done in various ways including:

- Measurement at a point,

- Measurement over a short section (around $10 \mathrm{~m}$ ),

- Measurement over a length of road (around $0.5 \mathrm{Km}$ ),

- The use of an observer moving in the traffic stream, and

- Wide-area samples obtained simultaneously from a number of vehicles.

Measurement at a point, which is the prevalent technique used by FRIEND, has been the first procedure used for traffic data collection. This method is easily capable of providing volume counts and therefore flow rates directly, and with care can also provide time headway. The most commonly used point detectors are based on inductive loop technology, but other methods in use include microwave, radar, photo-cells, ultrasonics, and television cameras. In general, traffic streams are not uniform, but vary over both space and time. Because of that, measurement of the variables of interest for traffic flow theory is in fact the sampling of a random variable. In reality, the traffic characteristics such as flow, speed, and concentration are parameters of statistical distributions, not absolute numbers [145].

\subsubsection{TRAFFIC FLOW PARAMETERS}

The traffic stream includes a combination of driver and vehicle behavior. The driver or human behavior being non-uniform, the traffic stream is also non-uniform in nature. It is influenced not only by the individual characteristics of both vehicle and human but also by the way a group of such units interacts with each other. Thus a flow of traffic through a street of defined characteristics will vary both by location and time corresponding to the changes in the human behavior [146]. From the traffic engineers' point of view, for the purpose of planning and design, the assumption is that these changes are within certain 
ranges which can be predicted. For example, if the maximum permissible speed of a highway is $\mathbf{5 5} \mathbf{~ m p h}$, the whole traffic flow can be assumed to move at an average speed of $45 \mathrm{mph}$. The following traffic flow parameters are essential to FRIEND:

- Speed: Speed is considered as a quality measurement of travel as the drivers and passengers will be concerned more about the speed of the journey than the design aspects of the traffic. It is defined as the rate of motion in distance per unit of time. Mathematically, speed, or velocity, $v$ is given by

$$
v=\frac{d}{t}
$$

where $v$ is the speed of the vehicle in $\mathrm{m} / \mathrm{s}, d$ is distance traveled in meters, and $t$ is time in seconds. The speeds of different vehicles will vary with respect to time and space. To represent these variations, several types of speed can be defined. Important among them are time mean speed and space mean speed [145]. We usually use time mean speed $v_{t}$, which is the arithmetic mean of speed of a group of vehicles passing a point. In this thesis, we also refer to space mean speed $v_{s}$, which is the harmonic mean of the speeds of vehicles passing a point during a period of time. Time mean speed is defined as the average speed of all the vehicles passing a point on a highway over some specified time period. Space mean speed is defined as the average speed of all the vehicles occupying a given section of a highway over some specified time period. Both mean speeds will always be different from each other except in the unlikely event that all vehicles are traveling at the same speed. Time mean speed is a point measurement, while space mean speed is a measure relating to length of highway or lane, i.e. the mean speed of vehicles over a period of time at a point in space is time mean speed and the mean speed over a space at a given instant is the space mean speed [146];

- Flow rate: There are practically two ways of counting the number of vehicles on a road. One is flow, or volume, which is defined as the number of vehicles that pass a point on a highway or a given lane or direction of a highway during a specific time interval [146]. The measurement is carried out by counting the number of vehicles, $n_{t}$, passing a particular point in one lane in a defined period $t$. Then the flow $q$ expressed in vehicles/hour is given by

$$
q=\frac{n_{t}}{t}
$$


In FRIEND, we consider that the variation of flow (volume) with time, i.e. month to month, day to day, hour to hour and within a hour, is also as important as the flow calculation. Flow variations can be observed from season to season. Volume will be above average in a pleasant motoring month of summer, but will be more pronounced in rural than in urban areas [146]. This is the most consistent of all the variations and affects the traffic stream characteristics the least. Weekdays, Saturdays and Sundays will also face differences in patterns. But comparing day with day, patterns for routes of a similar nature often show a marked similarity, which is useful in enabling predictions to be made. The most significant variation is from hour to hour. The peak hour is usually observed during mornings and evenings of weekdays, which is usually $8 \%$ to $10 \%$ of the total daily volume.

- Density: Density is defined as the number of vehicles occupying a given length of highway or lane and is generally expressed as vehicles per $\mathrm{Km} / \mathrm{mile}$. One can photograph a length of road $x$, count the number of vehicles, $n_{x}$, in one lane of the road at that point of time and derive the density $k$ as,

$$
k=\frac{n_{x}}{x}
$$

- Headway: We will refer to headway time $h_{t}$, which is the difference between the time when the front of a vehicle arrives at point on the highway and the time the front of the next vehicle arrives at the same point (in unit time). Also, we will refer to space headway $h_{s}$ as the difference in position between the front of a vehicle and the front of the next vehicle.

\section{Types of flow measurements}

Since there is considerable variation in the flow of traffic, several types of measurements of flow are commonly adopted which will average these variations into a single flow count to be used in many design purposes [146].

1. Average Annual Daily Traffic (AADT): The average 24-hour traffic flow at a given location over a full 365 -day year, i.e. the total number of vehicles passing the site in a year divided by 365 ;

2. Average Annual Weekday Traffic (AAWT): The average 24-hour traffic flow occurring on weekdays over a full year. It is computed by dividing the total weekday traffic flow for the year by 260 ; 
3. Average Daily Traffic (ADT): An average 24-hour traffic flow at given location for some period of time less than a year. It may be measured for six months, a season, a month, a week, or as little as one day. An ADT is a valid number only for the period over which it was measured;

4. Average Weekday Traffic (AWT): An average 24-hour traffic flow occurring on weekdays for some period of time less than one year, such as for a month or a season.

\section{Fundamental relations of traffic flow}

The relationship between the fundamental variables of traffic flow, namely, speed, flow, and density is referred to as the fundamental relations of traffic flow. They can be derived from a simple concept. We will use the following classic relation between flow, density and space mean speed

$$
q=k \times v_{s}
$$

\subsubsection{GREENSHIELD'S MODEL}

Greenshield [145] has developed a model of uninterrupted traffic flow that predicts and explains the trends observed in real traffic flows. Greenshield made the assumption that under uninterrupted flow conditions, speed and density are linearly related [145].

$$
v=A-B \times k
$$

where

$v$ is the speed (miles/hour, kilometers/hour);

$A, B$ are constants determined from field observations; and

$k$ represents the density (vehicles/mile, vehicles/kilometer).

We can determine the values of the constants $A$ and $B$ through field observations. This is normally done by collecting velocity and density data in the field, plotting the data, and then using linear regression to fit a line through the data points. The constant $A$ represents the free flow speed, while $\frac{A}{B}$ represents the jam density as shown in Figure 18. 


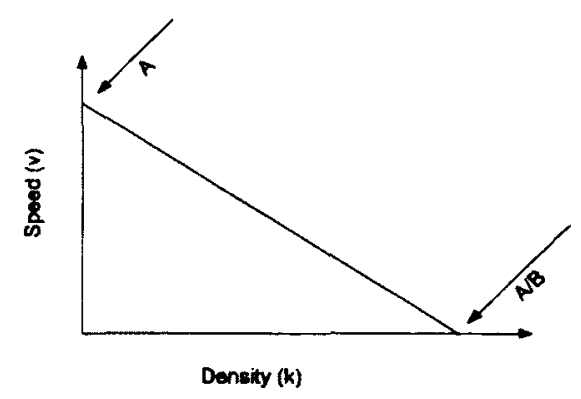

FIG. 18. Speed vs density.

Inserting Greenshield's speed-density relationship into the general speed-flow-density relationship yields the following equation

$$
\begin{aligned}
q & =(A-B \times k) \times k \\
& =A \times k-B \times k^{2},
\end{aligned}
$$

where

$q=$ flow (vehicles/hour)

$A, B=$ constants

$k=$ density (vehicles/mile, vehicles/kilometer)

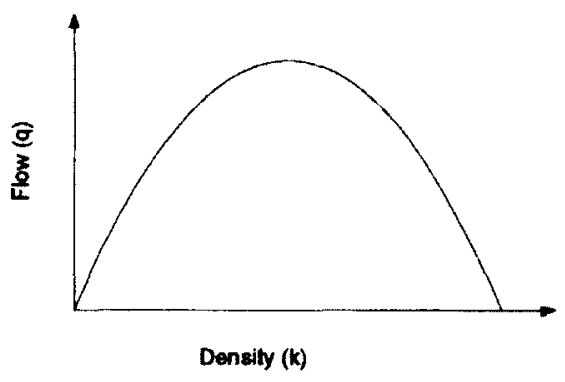

FIG. 19. Flow vs density.

This new relationship between flow and density, illustrated in Figure 19, provides an avenue for determining the density at which the flow is maximized 


$$
\frac{d q}{d k}=A-2 \times B \times k
$$

Now, setting $\frac{d q}{d k}=0$ yields

$$
k=\frac{A}{2 \times B} .
$$

Therefore, at the density given above, the flow will be maximized. Substituting this maximized value of $k$ into the original speed-density relationship yields the speed at which the flow is maximized.

$$
\begin{aligned}
v & =A B \times \frac{A}{2 \times B} \\
& =\frac{A}{2}
\end{aligned}
$$

This indicates that the maximum flow occurs when traffic is flowing at half of free-flow speed (A). Substituting the optimum speed and density into the speed-flow-density relationship yields the maximum flow.

$$
\begin{aligned}
q & =\frac{A}{2} \times \frac{A}{2 \times B} \\
& =\frac{A^{2}}{4 \times B}
\end{aligned}
$$

Figure 20 shows the relationship between flow and speed graphically.

As it turns out, Greenshield's model is quite powerful. The following corollaries can be derived from Greenshield's equations:

- When the density is zero, the flow is zero because there are no vehicles on the roadway;

- As the density increases, the flow also increases to some maximum flow conditions;

- When the density reaches a maximum, generally called jam density, the flow must be zero because the vehicles tend to line up end to end (parking lot conditions);

- As the density increases the flow increases to some maximum value, but a continual increase in density will cause the flow to decrease until jam density and zero flow conditions are reached. 


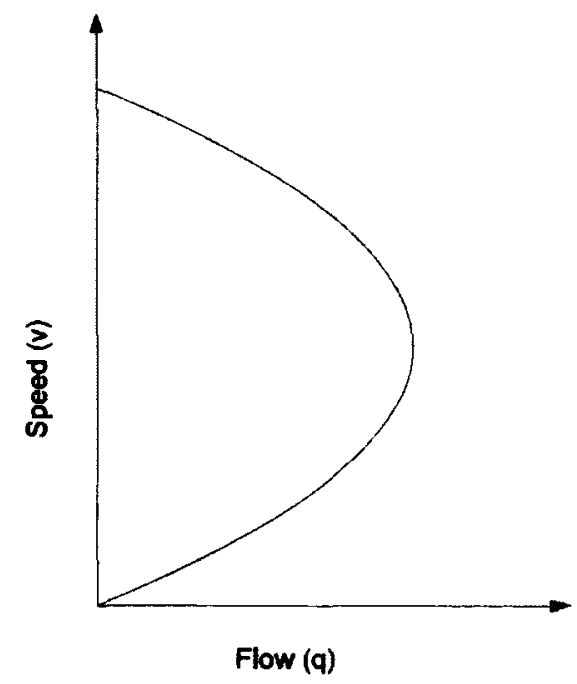

FIG. 20. Flow vs speed

In summary, the relationship between the speed and flow can be postulated as follows. The flow is zero either because there are no vehicles on the road or because there are too many vehicles so that they cannot move. At maximum flow, the speed will be in-between zero and free flow speed. The maximum flow $q_{\max }$ occurs at speed $u$. It is possible to have two different speeds for a given flow.

\subsection{MORE ON THE HEADWAY DISTANCE}

As already mentioned, the headway distance between consecutive cars on a roadway plays a fundamental role in describing the traffic flow. Not surprisingly, a great deal of effort has been devoted to understanding the various stochastic properties of the headway distance and, in the process, a number of headway distance models have been proposed in the literature.

In FRIEND, we define the headway distance as the distance separation between the fronts of two successive vehicles passing the same point on the highway. As will be discussed below, researchers have studied the headway in many traffic studies $[147,148,149]$. We note that in addition to the distance headway, some of these studies were concerned with time headway [147], which is the time interval between two vehicles passing a point as measured from the front bumper to the front bumper.

As pointed out by Cowan [150], typical representatives of such models include the 
exponential distribution, the normal distribution, the gamma distribution and the lognormal distribution. For instance, the log-normal distribution was proposed to model headways under car-following situations [151]. A major assumption for the log-normal headway model is that a vehicle maintains a safe distance while following its leading vehicle closely at variable speeds. This assumption makes sense and is apparent in real traffic data [152, 153, 154]. For example, Krbalek and Seba [154] studied the statistics of public transportation in and around Mexico-City. Chen and Li [155] proposed a Markov model to study the headway distance and concluded that the headway distance obeys a log-normal distribution. Chowdhury et al. [152] proposed a distribution of the headway distance as a function of the speed limit. The headways between two successive particles is defined based on the number of empty boxes between them. A similar study by Greenberg [156] reaches essentially the same conclusions. Panwai et al. [157] studied headway distance in microscopic mobility simulators as a car following model. Some mixed distribution models are proposed on the assumption that a road consists of two components, tracking/following and free components. For example, Cowan [150] proposed a mixed distribution consisting of a constant distribution (tracking/following component) and an exponential distribution (free component). Griffiths and Hunt [153] proposed a mixed model called Double Displaced Negative Exponential Distribution.

Given the large variety of opinions in the literature concerning the probability distribution of the headway distance, Yan and Olariu [4] recently validated these models in relation to their suitability as basis for analytical studies of link distribution in VANET. Towards this goal, they carried out experiments using the open source simulator written by Treiber [158]. Specifically, they have recorded and plotted the headway distance. Having plotted the resulting headway distance, they then plotted, on the same graph, the various candidate probability distributions just mentioned. As illustrated in Figure 21, Yan and Olariu [4] found that the best fit between a classic distribution function and the simulation results is provided by the log-normal distribution. It is interesting to mention that their conclusion agrees with a similar an experiment conducted independently by Puan [159]. Given the good fit between their simulation results shown in Figure 22, and Puan's data collected using video cameras to record traffic movement at four sites [159], in this thesis we adopt the log-normal distribution of headway distance as the basis for our results. 


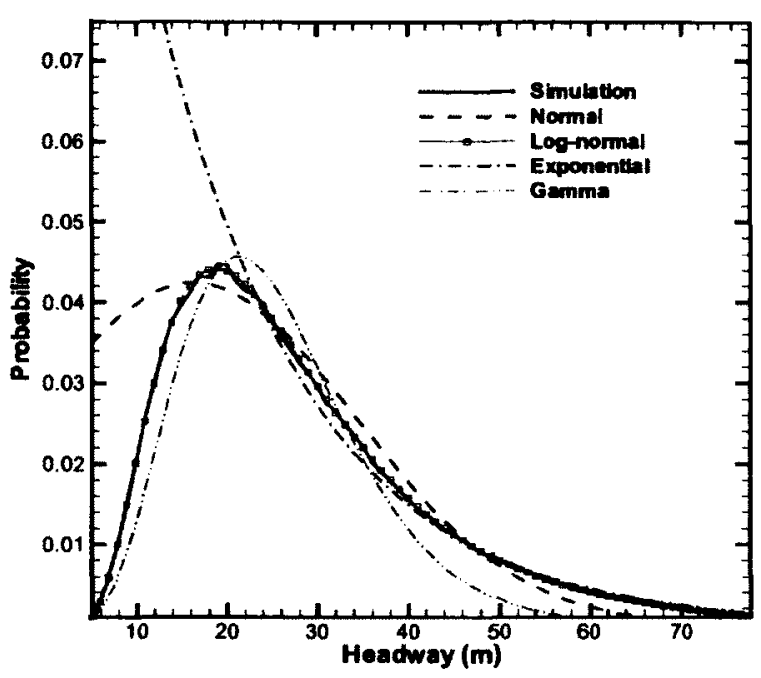

FIG. 21. The pdf of headway distance versus the normal, log-normal, exponential and gamma distribution (From [4]).

\subsubsection{THE PROBABILITY DISTRIBUTION OF A LINK}

\subsubsection{PATH LOSS}

The path loss model [160] is a radio propagation model that predicts the signal attenuation (in $\mathrm{dB}$ ) at a distance $X$ from the transmitter. Visser et al. [161] used a patch antenna and studied the pass loss of a DSRC link. The path loss of inter-vehicle communication can be modeled by two-ray model which takes the reflection signal from road into consideration. This suggests defining the path loss in $\mathrm{dB}$ as a random variable $L(X)$, a function of $X$ :

$$
L(X)=40 \log X-\left(10 \log G_{t}+10 \log G_{r}+20 \log h_{t}+20 \log h_{r}\right)
$$

where ${ }^{l} G_{t}$ and $G_{r}$ are the antenna gains of the transmitter and the receiver, respectively; $h_{t}$ and $h_{r}$ are, respectively, the heights of the transmitting and receiving antennas [160].

\subsubsection{LINK DISTANCE}

We are interested in link distance $X$, a distance between a sender and a receiver. Write

\footnotetext{
${ }^{1}$ Here, and in the remainder of this thesis, we use log to represent $\log _{10}$ and $\ln$ to represent the natural logarithm $\log _{e}$.
} 


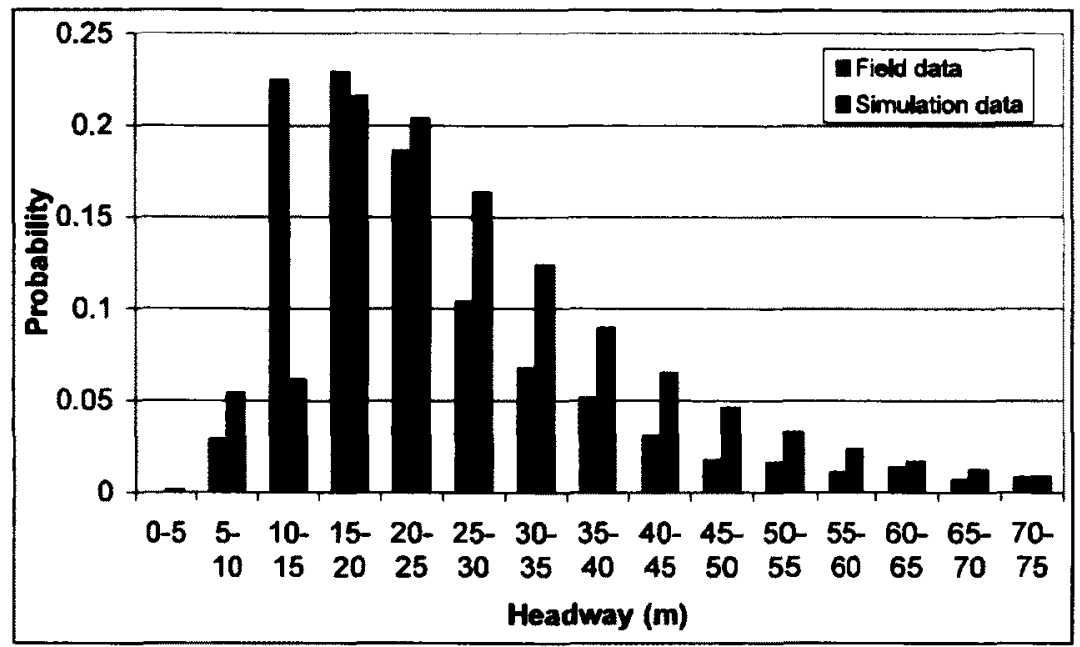

FIG. 22. Headway simulation vs. Puan's field data.

$X=\sum_{i=1}^{m} X_{i}$ where the $X_{i}$ s are independent log-normal random variables with a common distribution, specifically $X_{i} \in \log \mathrm{N}\left(\mu_{i}, \sigma_{i}\right)$. As illustrated in Figure 23, $X$ represents the convolution of $m$ independent headway distances. As it turns out, $X$ is approximately log-normal; the commonly-used Fenton-Wilkinson approximation [162] of $X$ is obtained by setting

$$
\begin{aligned}
& \sigma_{X}^{2}=\log \left[\frac{\left.\sum e^{2 \mu_{i}+\sigma_{i}^{2}\left(e_{i}^{2}\right.}+1\right)}{\left(\sum e^{\mu_{i}+\sigma_{i}^{2} / 2}\right)^{2}}+1\right] \\
& \mu_{X}=\log \left(\sum e^{\sigma_{i}^{2}}\right)-\frac{\sigma_{Z}^{2}}{2}
\end{aligned}
$$

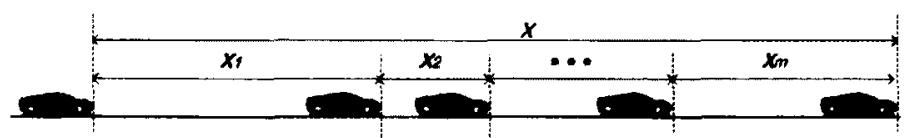

FIG. 23. Illustrating the convolution $X=X_{1}+X_{2}+\cdots+X_{m}$.

The headway distance can be calculated from the time headway by multiplying with 


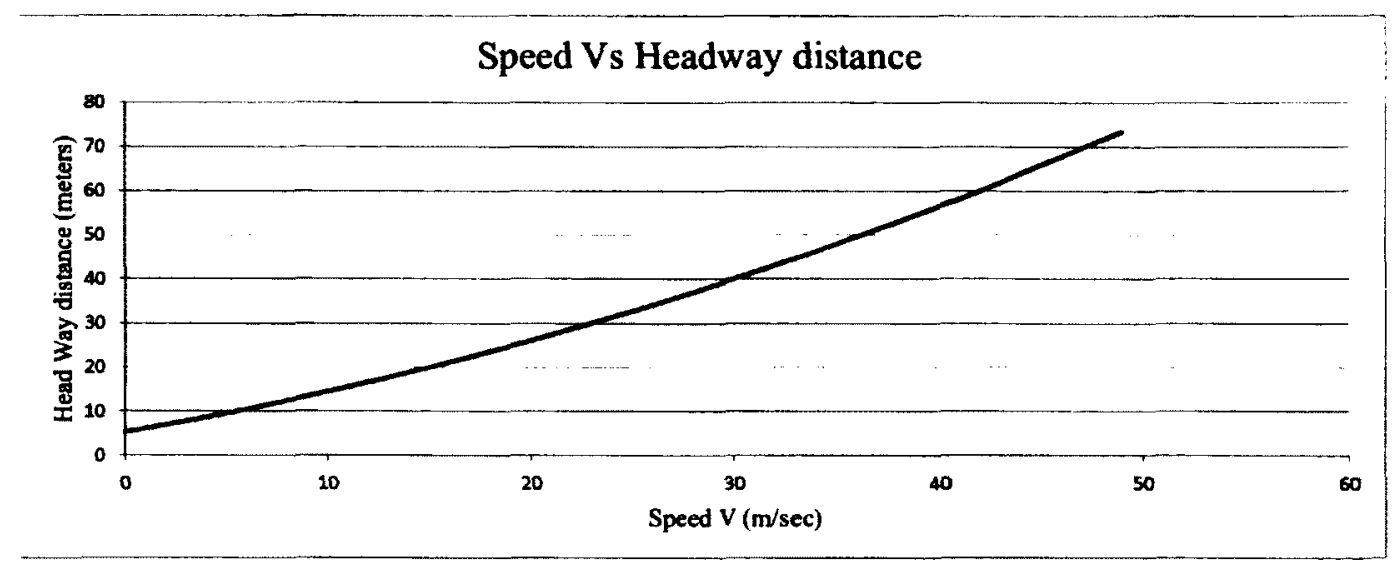

FIG. 24. Speed vs headway distance.

the speed, assuming a constant speed during the headway. A steady state headway distance has been derived in the form of

$$
H=5.34+0.792 V+0.01221 V^{2}
$$

where $H$ is the headway distance in meters and $V$ is the vehicle speed in $\mathrm{m} / \mathrm{sec}$. As mentioned above, Greenberg [156] was among the first to suggest that the distribution of the vehicle distance headway under constrained traffic situations is log-normal, where the standard deviation is dependent on the speed. The conclusion was derived from the assumption that drivers allow a constant reaction time and a constant buffer distance at all speeds. Figure 24 shows the plot of the relation between speed and headway distance from equation (20).

The mean headway distance in Greenberg study turns out to be

$$
H=1.37 \times V+10.338
$$

where the speed, $V$, is measured in $\mathrm{m} / \mathrm{s}$.

In general, the feasible range for speed and distance headway is illustrated in Figure 25 , where $l$ represents the minimum headway distance, defined as a vehicle's physical dimensions plus the minimum gap between two vehicles, and $V_{f}$ is the maximum freeflow speed. As a result, the headway distance increases as the speed increases. The points 


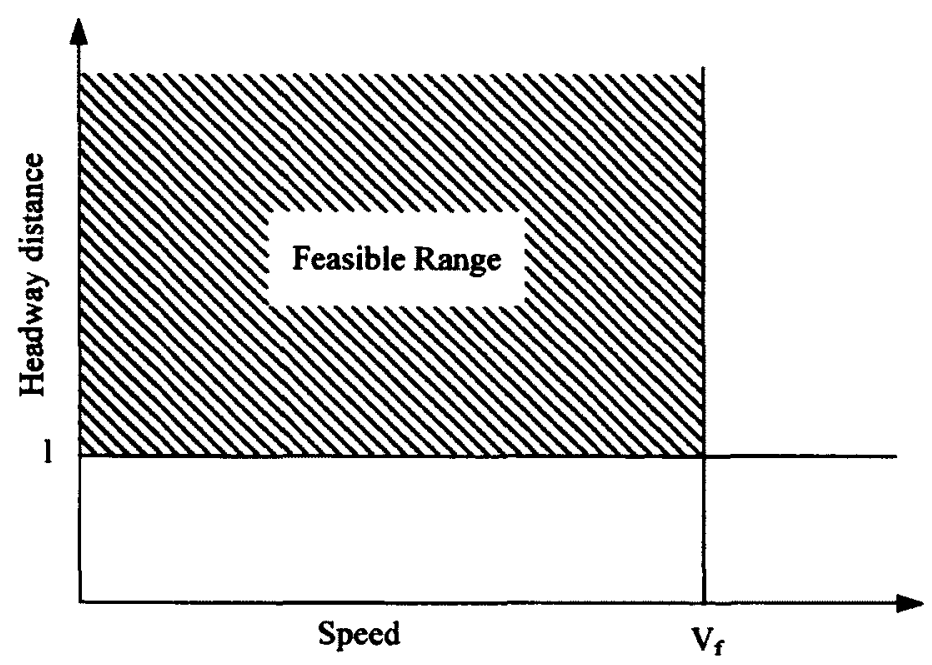

FIG. 25. Feasible range for speed and headway distance.

on the headway axis represent the stop condition with different densities, while the points on the $V$-axis represent the bumper to bumper situation. The hashed area bounded by the three lines represents a feasible range for speed and headway distance [163].

\subsubsection{SAFE HEADWAY DISTANCE}

In Figure 25, any point within the feasible range can present a possible vehiclefollowing condition. To avoid collision, the general distance headway is determined by travel speed and deceleration rate as mentioned in [163] as shown in equation:

$$
h_{g}=l+V \delta+\frac{V^{2}}{2 d_{2}}-\frac{V^{2}}{2 d_{1}}
$$

where

$l=$ vehicle length plus minimum gap between two successive vehicles,

$V=\operatorname{speed}(\mathrm{m} / \mathrm{s})$,

$d_{2}=$ following vehicle's deceleration rate $\left(\mathrm{m}^{2} / \mathrm{sec}\right)$, and

$d_{1}=$ leading vehicle's deceleration rate $\left(\mathrm{m}^{2} / \mathrm{sec}\right)$.

As noticed, all these pieces of information are given to the RSU while in communication with the vehicles. Safe distance headway varies with the speed and deceleration rates of the 
leading and following vehicles. The difference between two vehicle's deceleration rates results in different levels of safety. In general, a leading vehicle brakes harder than the following vehicle $\left(d_{1}<d_{2}\right)$ because the following vehicle can detect the situation by the brake lights of the leading vehicle. In case that leading vehicles stops instantly $\left(d_{1}=\infty\right)$, then the sufficient distance headway:

$$
h_{s}=l+V \delta+\frac{V^{2}}{2 d_{2}} .
$$

If the deceleration rates of both vehicles are the same, only the first terms will remain and this yields to minimum headway distance of

$$
h_{m}=l+V \delta .
$$

\subsection{REASONING ABOUT THE EXPECTED HEADWAY DISTANCE IN FREE-FLOW TRAFFIC}

Our next goal is to investigate clustering. It is customary to define a cluster in codirectional traffic as the collection of all the cars that have end-to-end DSRC connectivity. The motivation for this concept is clear: first, in terms of radio communications, messages can be routed from any one car in a cluster to any other car in the same cluster and to no car outside of the cluster. Second, in terms of traffic flow parameters, in conjunction with the headway distance, the size of a cluster (in a single lane) allows us to reason about traffic density.

We begin by an investigation of the expected headway distance in a single lane $L$ of co-directional traffic, assuming that the cars are distributed uniformly at random. The assumption about the cars being distributed uniformly is justified as follows. Assuming free-flowing traffic, it is natural to model the vehicle arrivals at an observer as a Poisson process with a certain parameter $\lambda$. Now, it is well known in probability theory that conditioned upon there being $k$ vehicles recorded in a time interval, these vehicles appear to be distributed uniformly at random along the lane.

Consider the lane $L$ partitioned into $n$ small slots, each the size of a hypothetical car, and assume that $k$ cars are placed, uniformly at random, in the $n$ slots. Let $X(j)$ be the random variable that denotes the slot in which we find the $j$-th car in left-to-right order. Theorem 4.3.1 calculates the expected headway distance for the $j$-th car.

Theorem 4.3.1 $E[X(j)]=j \cdot \frac{n+1}{k+1}$. 
Proof. By definition,

$$
E[X(j)]=\sum_{i=j}^{n-k+j} i \cdot p_{i, j}
$$

where $p_{i, j}$ is the probability that the $j$-th car occupies slot $i$.

To evaluate $p_{i, j}$, imagine that the $n$ slots are filled with $n$ objects, of which $k$ are cars and $n-k$ are some other objects. We concentrate on the number of permutations of the $n$ elements for which the $i$-th slot contains the $j$-th car. Indeed,

- the slots occupied by the leftmost $j-1$ cars can be selected out of the leftmost $i-1$ slots in $\left(\begin{array}{c}i-1 \\ j-1\end{array}\right)$ ways;

- once selected, these slots can be filled in $k(k-1) \cdots(k-j+2)=\frac{k !}{(k-j+1) !}$ ways;

- the remaining $i-j$ slots among the leftmost $i-1$ slots can be filled in $(n-k)(n-$ $k-1) \cdots(n-k-i+j+2)=\frac{(n-k) !}{[(n-i)-(k-j)] !}$ ways;

- the car to be placed in the $i$-th slot can be selected in $k-j+1$ ways;

- finally, the rightmost $n-i$ slots can be filled in $(n-i)$ ! ways.

It follows that

$$
\begin{aligned}
p_{i, j} & =\left(\begin{array}{l}
i-1 \\
j-1
\end{array}\right) \frac{k !}{(k-j) !} \cdot \frac{(n-k) !}{[(n-i)-(k-j)] !} \cdot \frac{(n-i) !}{n !} \\
& =\left(\begin{array}{l}
i-1 \\
j-1
\end{array}\right) \frac{k !(n-k) !}{n !} \frac{(n-i) !}{(k-j) ![(n-i)-(k-j)] !} \\
& =\left(\begin{array}{l}
i-1 \\
j-1
\end{array}\right) \cdot\left(\begin{array}{l}
n-i \\
k-j
\end{array}\right) \cdot\left(\begin{array}{l}
n \\
k
\end{array}\right)^{-1} \\
& =\frac{j}{i} \cdot\left(\begin{array}{l}
i \\
j
\end{array}\right) \cdot\left(\begin{array}{l}
n-i \\
k-j
\end{array}\right) \cdot\left(\begin{array}{l}
n \\
k
\end{array}\right)^{-1} .
\end{aligned}
$$

With this observation, $E[X(j)]$ can be written as 


$$
\begin{aligned}
E[X(j)] & =\sum_{i=j}^{n-k+j} i \cdot p_{i, j} \\
& =\sum_{i=j}^{n-k+j} j \cdot\left(\begin{array}{l}
i \\
j
\end{array}\right) \cdot\left(\begin{array}{l}
n-i \\
k-j
\end{array}\right) \cdot\left(\begin{array}{l}
n \\
k
\end{array}\right)^{-1} \\
& =j \cdot\left(\begin{array}{l}
n \\
k
\end{array}\right) \sum_{i=j}^{-1}\left(\begin{array}{l}
i \\
j
\end{array}\right) \cdot\left(\begin{array}{l}
n-i \\
k-j
\end{array}\right) \\
& =j \cdot\left(\begin{array}{l}
n \\
k
\end{array}\right)^{-1} \sum_{t=0}^{n-k}\left(\begin{array}{c}
n-j-t \\
k-j
\end{array}\right)\left(\begin{array}{c}
j+t \\
j
\end{array}\right) \\
& =j \cdot\left(\begin{array}{l}
n \\
k
\end{array}\right)^{-1} \sum_{t=0}^{n-j}\left(\begin{array}{c}
n-j-t \\
k-j
\end{array}\right)\left(\begin{array}{c}
j+t \\
j
\end{array}\right) \\
& =j \cdot\left(\begin{array}{l}
n \\
k
\end{array}\right)^{-1} \cdot\left(\begin{array}{c}
n+1 \\
k+1
\end{array}\right) \\
& =j \cdot \frac{(n+1) ! k !(n-k) !}{(k+1) !(n-k) ! n !} \\
& =j \cdot \frac{n+1}{k+1} .
\end{aligned}
$$

This completes the proof.

Even though intuitively satisfying, the result derived above is almost too good to be true. What can go wrong? For one thing, it is an easy exercise to show that in spite of the regularity suggested by the value of $E[X(j)]$, the variance of the headway distance is quite significant. With this in mind, our goal becomes to provide an answer to the following natural question: Given that $m$ cars are deployed uniformly at random in a single lane of traffic of one kilometer and given that dependable radio communications between cars requires a maximum inter-car distance of 200 meters $^{2}$ what is the probability that there is end-to-end radio connectivity between the $m$ cars? This question is fundamental since virtually all proponents of multihop routing algorithms involving co-directional cars in VANET take end-to-end-connectivity for granted. We prove that, quite surprisingly, the number of cars per kilometer must be at least 16 in order to have a better than even chance for connectivity. Moreover, it takes about 25 cars per kilometer for end-to-end connectivity to be present with $90 \%$ probability.

\footnotetext{
${ }^{2}$ While the transmission range in DSRC is stipulated to be a minimum of $300 \mathrm{~m}$, several recent studies have shown that at more than $200 \mathrm{~m}$ packet reception is unreliable. With this in mind, we set out to study reliable end-to-end connectivity, assuming that the maximum transmission range is only $200 \mathrm{~m}$.
} 


\subsubsection{EVALUATING THE PROBABILITY OF LARGE HEADWAY DISTANCES IN CO-DIRECTIONAL TRAFFIC}

The goal of this subsection is to provide an answer to the following natural question: Given that $m$ cars are deployed uniformly at random in a single lane of traffic of one kilometer and given that dependable radio communications between cars require a maximum inter-car distance of $\mathbf{2 0 0}$ meters, what is the probability that there is end-to-end radio connectivity between the $m$ cars? We prove that, surprisingly, the number of cars per kilometer must be at least $\mathbf{1 6}$ in order to have a better than even chance for connectivity; it takes about 25 cars per kilometer for end-to-end connectivity to be present with $90 \%$ probability.

We model the situation as follows: the $m$ cars determine $m-1$ distinguishable bins (inter-car spaces), enumerated in left-to-right order as $B_{1}, B_{2}, \ldots B_{m-1}$. The number of distinguishable ways in which the $n$ indistinguishable balls (unit inter-car spaces) can be distributed into the $m-1$ bins is easily seen to be $\left(\begin{array}{c}m+n-2 \\ n\end{array}\right)=\left(\begin{array}{c}m+n-2 \\ m-2\end{array}\right)$. To see that this is the case, observe that the $m-1$ bins involve $m$ separators and that we can lay down the balls and bins in a linear sequence flanked on both sides by a separator. The problem now is that of selecting $n$ places for the balls out of a total of $n+m-2$ places available. The conclusion follows.

Now suppose that we want a given bin to contain $k,(0 \leq k \leq n)$, balls. This amounts to distributing $k$ balls into one bin and $n-k$ balls into the remaining $m-2$ bins. Reasoning as above, the number of distinguishable ways in which this can be achieved is $\left(\begin{array}{c}(n-k)+(m-3) \\ n-k\end{array}\right)=\left(\begin{array}{c}n+m-k-3 \\ n-k\end{array}\right)$. Thus, the probability $p_{k},(0 \leq k \leq n)$, of the event that a given bin contains exactly $k$ balls is

$$
p_{k}=\left(\begin{array}{c}
n+m-k-3 \\
n-k
\end{array}\right)\left(\begin{array}{c}
m+n-2 \\
n
\end{array}\right)^{-1} .
$$

To show that the $p_{k} s$ are a valid probability distribution, we need to prove that $\sum_{k=0}^{n} p_{k}=1$. This, in turn, amounts to showing that $\sum_{k=0}^{n}\left(\begin{array}{c}(n-k)+(m-3) \\ n-k\end{array}\right)=\left(\begin{array}{c}m+n-2 \\ n\end{array}\right)$. Recalling that for integers $r$ and $n$,

$$
\sum_{t \leq n}\left(\begin{array}{c}
r+t \\
t
\end{array}\right)=\left(\begin{array}{c}
r+n+1 \\
n
\end{array}\right)
$$


we write

$$
\begin{aligned}
\sum_{k=0}^{n} p_{k} & =\left(\begin{array}{c}
m+n-2 \\
n
\end{array}\right)^{-1} \sum_{k=0}^{n}\left(\begin{array}{c}
(n-k)+(m-3) \\
n-k
\end{array}\right) \\
& =\left(\begin{array}{c}
m+n-2 \\
n
\end{array}\right)^{-1} \sum_{i \leq n}\left(\begin{array}{c}
(m-3)+i \\
i
\end{array}\right) \\
& =\left(\begin{array}{c}
m+n-2 \\
n
\end{array}\right)^{-1}\left(\begin{array}{c}
(m-3)+n+1 \\
n
\end{array}\right) \text { [by (28)] } \\
& =\left(\begin{array}{c}
m+n-2 \\
n
\end{array}\right)^{-1}\left(\begin{array}{c}
m+n-2 \\
n
\end{array}\right) \\
& =1
\end{aligned}
$$

as desired.

Observe that two neighboring vehicles become disconnected if the bin corresponding to the distance between them accumulates at least $d+1$ balls, where $d$ corresponds to the maximum effective transmission range. Let $A_{i},(1 \leq i \leq m-1)$, be the probability that a generic bin $B_{i}$ contains at least $d+1$ balls.

Lemma 4.3.2 For all $i,(1 \leq i \leq m-1)$,

$$
\operatorname{Pr}\left[A_{i}\right]=\left(\begin{array}{c}
m+n-(d+1)-2 \\
m-2
\end{array}\right)\left(\begin{array}{c}
m+n-2 \\
n
\end{array}\right)^{-1}
$$

Proof. Let us compute the probability of $\bar{A}_{i}$. By (27) and (28) we can write

$$
\begin{aligned}
\operatorname{Pr}\left[\bar{A}_{i}\right] & =\left(\begin{array}{c}
m+n-2 \\
n
\end{array}\right)^{-1} \sum_{j=0}^{d}\left(\begin{array}{c}
(m-3)+(n-j) \\
n-j
\end{array}\right) \\
& =\left(\begin{array}{c}
m+n-2 \\
n
\end{array}\right)^{-1} \sum_{t=n-d}^{n}\left(\begin{array}{c}
(m-3)+t \\
t
\end{array}\right) \\
& =\left(\begin{array}{c}
m+n-2 \\
n
\end{array}\right) \sum_{t=0}^{-1}\left(\begin{array}{c}
(m-3)+t \\
t
\end{array}\right) \\
& -\left(\begin{array}{c}
m+n-2 \\
n
\end{array}\right)^{-1} \sum_{t=0}^{n-d-1}\left(\begin{array}{c}
(m-3)+t \\
t
\end{array}\right) \\
& =1-\left(\begin{array}{c}
m+n-2 \\
n
\end{array}\right)^{-1}\left(\begin{array}{c}
m+n-d-3 \\
m-2
\end{array}\right)
\end{aligned}
$$


Thus, $\operatorname{Pr}\left[A_{i}\right]=1-\operatorname{Pr}\left[\bar{A}_{i}\right]=\left(\begin{array}{c}m+n-2 \\ n\end{array}\right)^{-1}\left(\begin{array}{c}m+n-d-3 \\ m-2\end{array}\right)$, and the proof of the lemma is complete.

Let $A$ be the event that there is no end-to-end connectivity between the $m$ cars. Clearly, $A=\cup_{i=1}^{m-1} A_{i}$. Since the $A_{i} \mathrm{~s}$ are not independent, the principle of inclusionexclusion implies that $\operatorname{Pr}[A]=\sum_{i=1}^{m-1} \operatorname{Pr}\left[A_{i}\right]-\sum_{1 \leq i<j \leq m-1} \operatorname{Pr}\left[A_{i} \cap A_{j}\right]+\cdots+$ $(-1)^{i} \sum_{1 \leq j_{1}<j_{2}<\ldots<j_{i} \leq m-1} \operatorname{Pr}\left[A_{j_{1}} \cap A_{j_{2}} \cap \cdots \cap A_{j_{i}}\right]+\cdots$

Lemma 4.3.3 For all $i, j,(1 \leq i<j \leq m-1), \sum_{1 \leq i<j \leq m-1} \operatorname{Pr}\left[A_{i} \cap A_{j}\right]=$ $\left(\begin{array}{c}m-1 \\ 2\end{array}\right)\left(\begin{array}{c}m+n-2(d+1)-2 \\ m-2\end{array}\right)\left(\begin{array}{c}m+n-2 \\ n\end{array}\right)^{-1}$.

Proof. Observe that the number of distinguishable arrangements in which bins $i$ and $j$ contain at least $d+1$ balls is obtained by first placing $d+1$ balls in bins $i$ and $j$ and then by distributing the remaining $n-2(d+1)$ balls uniformly at random in all the $m-1$ bins. This can be done in $\left(\begin{array}{c}m+n-2(d+1)-2 \\ n-(d+1)\end{array}\right)=\left(\begin{array}{c}m+n-2(d+1)-2 \\ m-2\end{array}\right)$ distinct ways. Since there are $\left(\begin{array}{c}m-1 \\ 2\end{array}\right)$ distinct ways of choosing $i$ and $j$ subject to $(1 \leq i<j \leq m-1)$, the conclusion follows.

In a perfectly similar way we can prove the following companion result.

Lemma 4.3.4 For all $1 \leq j_{1}<j_{2}<\ldots<j_{i} \leq m-1, \sum_{1 \leq j_{1}<j_{2}<\ldots<j_{i} \leq m-1} \operatorname{Pr}\left[A_{j_{1}} \cap\right.$ $\left.A_{j_{2}} \cap \cdots \cap A_{j_{i}}\right]=\left(\begin{array}{c}m-1 \\ i\end{array}\right)\left(\begin{array}{c}m+n-i(d+1)-2 \\ m-2\end{array}\right)\left(\begin{array}{c}m+n-2 \\ n\end{array}\right)^{-1}$.

The following important result follows from Lemmas 4.3.2, 4.3.3 and 4.3.4.

\section{Theorem 4.3.5}

$$
\operatorname{Pr}[A]=\frac{\sum_{i=1}^{m-1}(-1)^{i+1}\left(\begin{array}{c}
m-1 \\
i
\end{array}\right)\left(\begin{array}{c}
m+n-i(d+1)-2 \\
m-2
\end{array}\right)}{\left(\begin{array}{c}
m+n-2 \\
n
\end{array}\right)} .
$$

One can interpret (29) as follows. Imagine sliding a $1 \mathrm{Km}$ window down an undivided roadway with one lane of traffic in each direction. If the window contains $m$ co-directional cars, then the probability that there is no end-to-end connectivity between them is precisely $\operatorname{Pr}[A]$ in (29). For example, if there were 12 co-directional cars in the window, the probability of no end-to-end connectivity between them would be about $86 \%$. Naturally, the probability decreases with the number of co-directional lanes of traffic in each direction.

\subsubsection{EVALUATING THE EXPECTED SIZE OF A CLUSTER}

Since, as we saw, co-directional traffic is inherently partitioned into clusters, each consisting of all the cars enjoying end-to-end connectivity, an interesting question is to estimate the expected size of a cluster. The goal of this subsection is to provide an answer 
to this natural question. For this purpose, we inherit the notation and terminology of the previous subsection.

Theorem 4.3.6 The expected size of a cluster is

$$
E[\text { cluster_size }]=\frac{m \cdot\left(\begin{array}{c}
m+n-2 \\
n
\end{array}\right)}{\left(\begin{array}{c}
m+n-2 \\
n
\end{array}\right)+(m-1) \cdot\left(\begin{array}{c}
m+n-d-3 \\
n
\end{array}\right)} .
$$

Proof. As we saw, the probability $p$ that a given bin contains at least $d+1$ balls is $p=\left(\begin{array}{c}m+n-d-3 \\ m-2\end{array}\right)\left(\begin{array}{c}m+n-2 \\ n\end{array}\right)^{-1}$. Let $X$ be the random variable that counts the number of "gaps" (i.e., the number of bins containing at least $d+1$ balls). Since $X$ is binomial, the expected value $E[X]$ of $X$ is

$$
\begin{aligned}
E[X] & =(m-1) \cdot p \\
& =(m-1) \cdot\left(\begin{array}{c}
m+n-d-3 \\
m-2
\end{array}\right)\left(\begin{array}{c}
m+n-2 \\
n
\end{array}\right)^{-1}
\end{aligned}
$$

Once we have the expected number of gaps in co-directional traffic, the expected number of clusters becomes $1+E[X]=1+(m-1) \cdot\left(\begin{array}{c}m+n-d-3 \\ m-2\end{array}\right)\left(\begin{array}{c}m+n-2 \\ n\end{array}\right)^{-1}$. Thus, the expected size of a cluster is

$$
E[\text { cluster_size }]=\frac{m \cdot\left(\begin{array}{c}
m+n-2 \\
n
\end{array}\right)}{\left(\begin{array}{c}
m+n-2 \\
n
\end{array}\right)+(m-1) \cdot\left(\begin{array}{c}
m+n-d-3 \\
n
\end{array}\right)},
$$

completing the proof of the theorem.

Traffic flow on highways tends to be disconnected. The cluster size can help approximating density on the highway using the Average Headway Distance (AHD) calculated by each RSU. Researchers proved that, somewhat surprisingly, the cluster size is quite stable and easy to maintain [164]. In [164], the authors show that the expected size of a cluster is a function of the density and the communication range. We track the number of vehicles in a road segment of one kilometer and the size of the cluster. We ran a simulator that calculated the number of clusters that is created in a $1 \mathrm{Km}$ length (i.e. the expected distance between two adjacent RSUs) and compared it to the number of vehicles in the segment as shown in Figure 26.

\subsection{LEARNING TRAFFIC FLOW PARAMETERS}




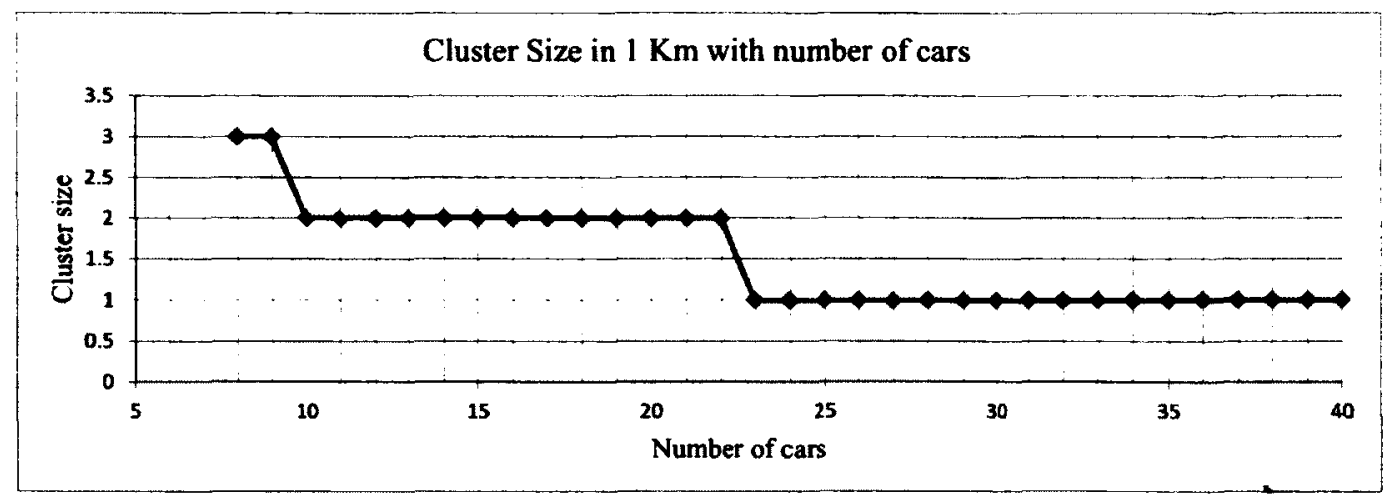

FIG. 26. Number of clusters vs number of vehicles in $1 \mathrm{Km}$.

It is important to mention that it is a non-trivial task for FRIEND to learn the state of the traffic parameters from passing vehicles only. One of the valuable tools used are lessons learned from the theoretical results in Section 4.3. However, it is important to bear in mind that the above derivations are modeling a simple scenario and that, as any mathematical model, they can only approximate the real world.

For example, it is natural to employ sampling techniques to evaluate the headway distance. We discuss the parameters that changes the flow between states. In order to aggregate the data collected from the passing cars and, if necessary, from the SCEs, the RSUs keep track of three main parameters:

- Historical data: The RSUs have a record of time of the day, day of the week and flow record which is a function of the speed, headway and density. This record of historical data is the single most important ingredient in deciding about the current status of the traffic. For example, if the most recent batch of collected data, suitably thresholded, is within acceptable tolerance from the collected historical data, then the traffic is deemed to be normal and no special action needs to be undertaken. If however, a significant deviation from the accumulated historical data is detected then the RSU concludes that the traffic is not normal and further action is necessary as will be discussed in Chapter 6;

- Speed: The RSUs, while exchanging information with vehicles, record the previous segment's average speed. Then, they calculate the average speed of the passing vehicles; 
- Headway distance: The RSUs keep track of the average headway distance of the passing vehicles. This can be done through two methods:

1. Method A: From data recorded in vehicles, each vehicle keeps track of the headway distance of the front vehicles using its radar;

2. Method B: From (time, location, lane, speed) where data exchange occurs as shown below in Figure 27.

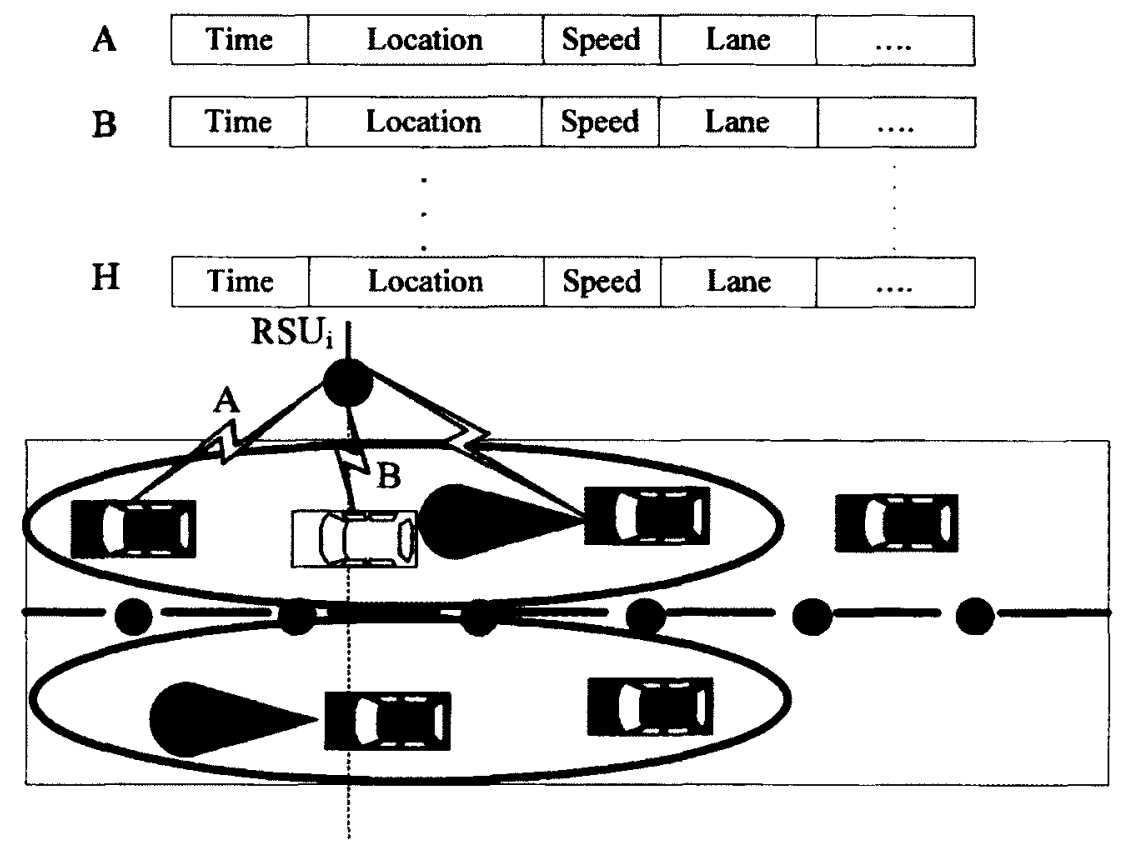

FIG. 27. Headway computation by RSUs.

We now discuss our strategy for determining and recording the average headway distance of vehicles. Each RSU maintains a buffer that keeps the most recent recorded headway distance. If a change occurs in the values of the headway distance, an update to the buffer is needed. In case of rapidly changing traffic (i.e. a cluster of vehicles are passing), an increase in the size of the buffer is done. The following are the steps performed by the RSU to calculate the average headway distance:

1. $R S U_{i}$ (in time period $\left[t_{0}, t_{1}\right]$ ) receives number of records from different vehicles, each record includes time, location, speed and lane; 
2. $R S U_{i}$ calculate the location of each vehicle at time $T$ within same lane;

3. $R S U_{i}$ sorts the records and calculates the headway distance between each vehicle;

4. $R S U_{i}$ updates headway buffer with headway distances recorded;

5. $R S U_{i}$ compares the headway in the buffer with any received headway data from vehicles;

6. the recorded data in the buffer can give us an indication of the traffic density on the highway at the $R S U_{i}$.

\subsection{SUMMARY}

This chapter started with background on traffic flow parameters. Then, we discussed type of flow measurements and Greenshield's model for traffic flow. Then, we showed our idea of reasoning about headway distance by explaining the probability distribution of a link and defining the safe beadway distance concept. We calculated the expected headway distance in free-flow traffic and the probability of large headway distance in a co-directional traffic. Then, we showed how to evaluate the expected cluster size. Finally, we explained the steps to track the three main parameters in FRIEND: the historical data, speed, and headway distance. 


\section{CHAPTER 5}

\section{COMMUNICATION PROTOCOLS IN FRIEND}

The main goal of this chapter is to discuss the communication protocols employed by various entities in FRIEND. We discuss one of the most fundamental issues in computer networking, which is how two entities can reliably communicate. In Section 5.1, we start by introducing notation and by establishing terminology that will be used throughout the remainder of the thesis. In Section 5.2, we discuss how the RSUs communicate with different other entities in FRIEND. In Section 5.3, we explain the communication between SCEs and other FRIEND entities, then we give some other definitions. Finally, for later reference, we explain what is meant by the Head and Tail of a traffic backup or incident.

\subsection{DEFINITIONS}

Definition 5-1: RSU-RSU distance The distance between two adjacent RSUs is called RSU-RSU distance. Further, $R S U-R S U[i, j]$ is the distance between $R S U_{i}$ and $R S U_{j}$ where $i<j$ as shown in Figure 28.

Definition 5-2: Segment The distance between two adjacent RSUs is divided into a number of segments, each segment is named Segment $[i, j, n u m]$ where $i<j$ refer to the number of $R S U-R S U[i, j]$. num is the segment number. Each segment contains a number of SCEs.

\subsection{RSU COMMUNICATION}

The RSUs are the entities responsible for communicating with both the vehicles and with other SCEs and neighboring RSUs. As it turns out, in FRIEND, the RSUs play a key role in data collection, in processing the map algorithm, and in the dissemination and propagation of traffic information, to be discussed in detail in Chapter 6.

\subsubsection{COMMUNICATION BETWEEN ADJACENT RSUS}


RSU-RSU[i,i+1]

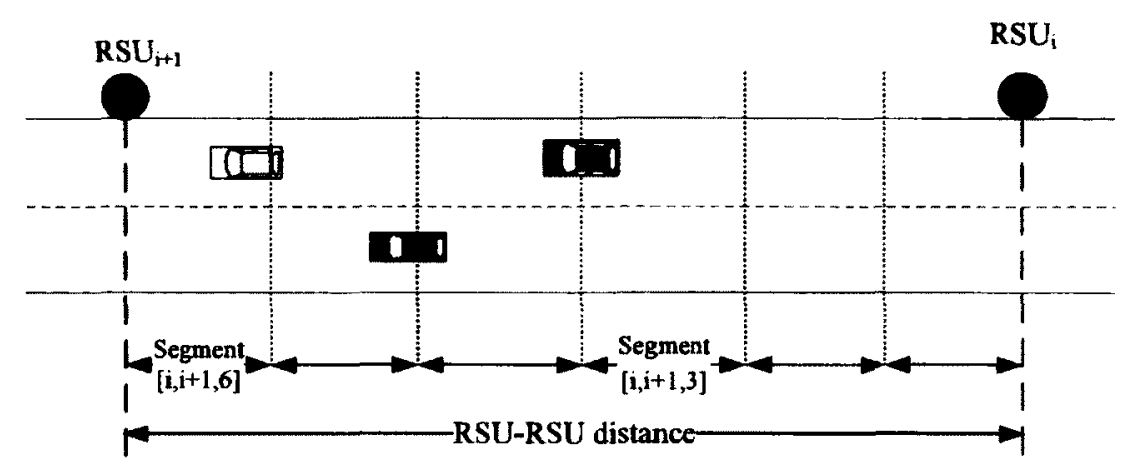

FIG. 28. Segment and RSU-RSU[i,j] definition

Under normal traffic conditions, adjacent RSUs along the roadway do not communicate with each other directly, relying instead on passing cars to act as couriers carrying non time-critical information between them. However, whenever time-critical messages need to be exchanged, adjacent RSUs can, and do, communicate directly for transient periods of time using some form of radio communications, e.g. a DSRC radio interface that covers distances up to $1 \mathrm{~km}$.

In order to make the communication between adjacent RSUs secure, each adjacent pair, say $A$ and $B$, of RSUs along the roadway (see Figure 29) shares a time-varying symmetric key $\mu(A, B, t)$ used to encrypt, at time $t$, the data exchanged between them. Since the RSUs are synchronous (by virtue of the GPS), they switch from one key to the next in a pre-established order based on their local time.

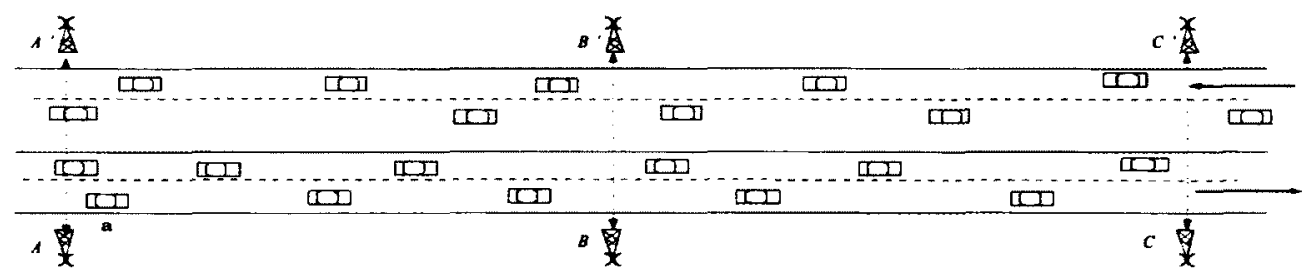

FIG. 29. Illustrating non-time critical communication between adjacent RSUs.

Referring to Figure 29, assume that RSU $A$ has a non time-critical message $m$ for RSU $B$. $A$ will encrypt $m$ with $\mu(A, B, t)$ and will upload it onto passing car $a$. When car $a$ 
reaches $B$, the message $m$ will be dropped off by car $a$ and decoded by $B$. In turn, $B$ may decide to send a message to RSU $C$. This would be done using the symmetric key $\mu(B, C, t)$, known only to $B$ and $C$. It is important to note that, given a sufficiently large set of keys in the key-chain, the RSU to RSU encryption keys appear random to an external observer. A key component of FRIEND is a robust scheme that allows adjacent RSUs to communicate securely.

Occasionally, adjacent RSUs need to communicate to corroborate sudden changes in the basic parameters of the traffic flow that they (or one of them) experience(s). Such is the case, for example, in the initial stages of congestion or when an incident occurring in the segment between them triggers changes in the traffic flow. Under such conditions, adjacent RSUs communicate directly. In the case of an incident, the first key issue that the RSUs need to determine is a coarse-grained determination of the location of the incident to the granularity of a RSU-to-RSU segment. This task is done as follows: every RSU that has detected an abnormal drop in traffic (relative to historical data) sends a query to its adjacent neighbors. This allows to identify a unique pair of RSUs such that (with high probability) the incident (assuming there is one) has occurred in the road segment flanked by them. The second key task involves the fine-grained determination of the location of the accident, to the granularity of the distance $d$ between adjacent SCEs.

A third type of communication between adjacent RSUs is in support the propagation of the color-coded traffic status reports to vehicles along the roadway. We anticipate that this kind of communication is low data rate and will involve sending, once a minute or so, an aggregated packet containing the local traffic view of a group of about ten consecutive RSUs.

\subsubsection{RSU COMMUNICATION WITH VEHICLES}

As mentioned in Chapter 3, the RSUs exchange data with passing vehicles. A car approaching a RSU is either entitled to drop off EDR data with the RSU or else it is considered "new" and is not allowed to do so. Indeed, cars that have completed a handshake with the previous RSU have received a one-time session key $\alpha$ that entitles them to drop off their EDR data upon correctly handshaking with the next RSU. Vehicles that either have just entered the roadway or have failed to handshake with the previous RSU are considered "new" and are not entitled to drop off EDR information with the RSU. Since the RSUs are synchronized, a RSU can easily validate an alleged session-key $\alpha$. In effect, using onetime session keys issued by the previous RSU precludes cars (including those stationed 
by the roadside) from mounting a Sybil attack on the RSU. Also, the session key is independent of the identity of the vehicle allowing for privacy-preserving communications between vehicles and RSUs.

Referring to Figure 30, a connection between a vehicle and a RSU is established only when the vehicle enters the RSU's coverage area and receives, on a control channel $\lambda$, a beacon containing handshaking information. The vehicle replies with a "HELLO" message containing either $\lambda$ encrypted with the one-time key $\alpha$, or contains only $\lambda$ if the car is "new" and does not possess a key $\alpha$. The RSU then replies with an ACK message either encrypted with $\alpha$ or else issues to the car a one-time session key $\beta$ representing the exact contact time encrypted with the symmetric key between the current and next RSU down the road.

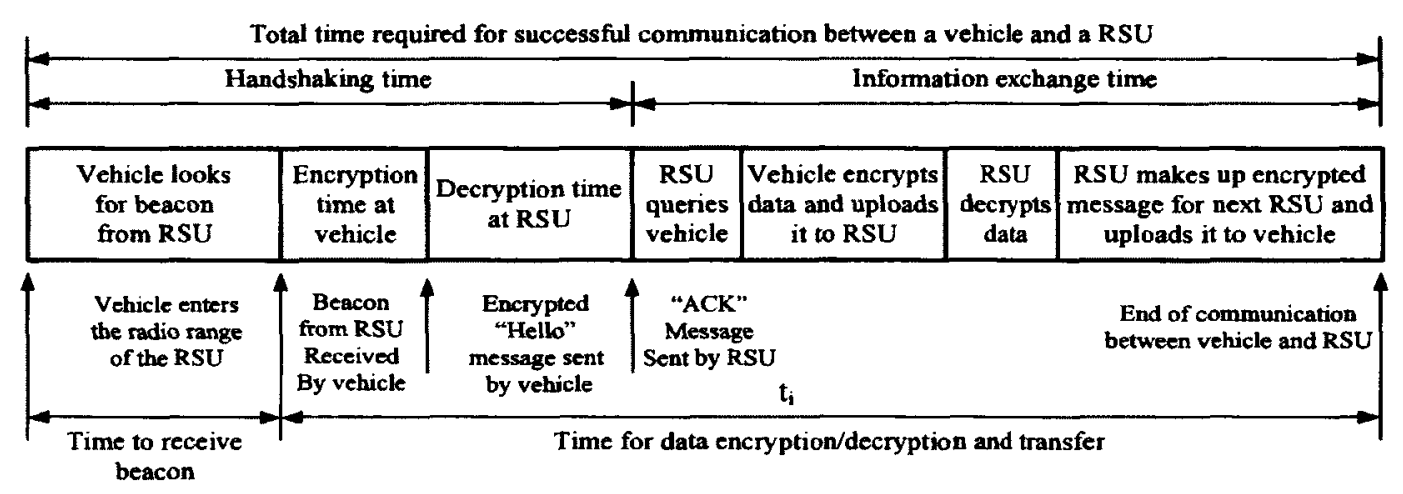

FIG. 30. Illustrating the details of vehicle to RSU communication.

Let us follow a hypothetical car that was issued a one-time key $\beta$. When this car reaches the next RSU, it will present the RSU with $\beta$ and, upon successful validation, will be allowed to drop off data collected by its EDR. As a precautionary measure, the RSU verifies that the time at which the car passed the previous RSU as recorded by its EDR corresponds to the value $\beta$ properly decrypted. If the two match, the credentials are accepted and the data exchange proceeds. Otherwise, the credentials are rejected and the car is, again, considered "new" and is not allowed to drop off EDR data.

Consider now a car whose one-time session key $\alpha$ is recognized as valid. The ACK message returned by the RSU contains a random frequency channel $\sigma$ (encrypted with $\alpha$ ) on which subsequent data exchange is to take place; in addition, a secure one-time key may be established for the purpose of the data exchange. The car switches to channel 


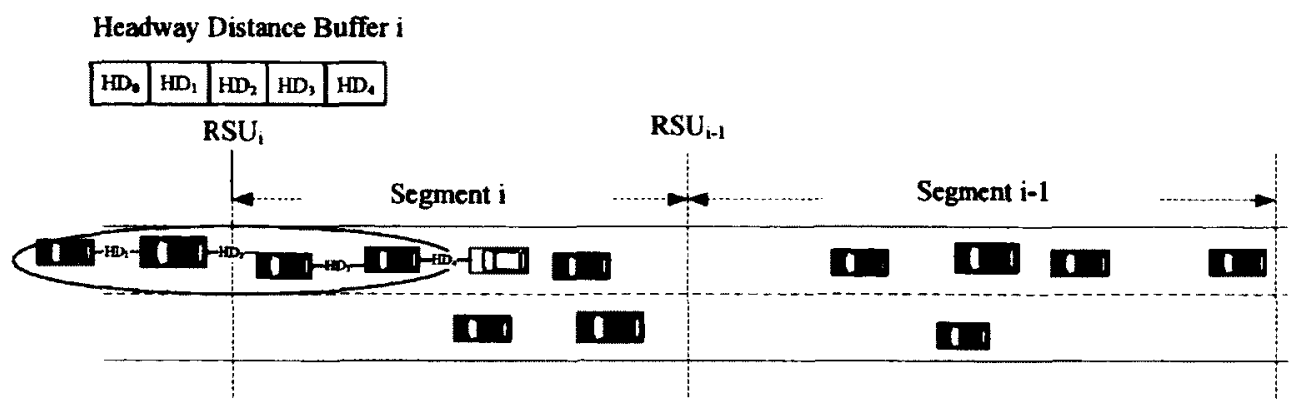

FIG. 31. Illustrating the headway distance buffer at $R S U_{i}$

$\sigma$ and proceeds to the data exchange with the RSU by: (1) sending the data in its EDR (along with encrypted data from the previous RSU); and (2) downloading any notification and warning messages that the RSU has available. Furthermore, the data exchange between car and RSU requires that a wireless radio link be established and that data be successfully transmitted. The former requirement depends on the RSU beacon spacing (that is how often the beacon signal is sent by the RSU) and on the time required to encryption/decryption the "HELLO" message sent by the car. The latter requirement depends on the encryption/decryption times and the data rate of the wireless link.

\subsubsection{DATA COLLECTED AND EXCHANGED BETWEEN RSU AND VEHICLES}

As will be justified in detail in Chapter 6, each RSU maintains a headway buffer to save the headway distance over time only if the values are changing, which indicates a change in the density of the traffic.

\subsubsection{EVALUATING THE INSTANTANEOUS AVERAGE HEADWAY DISTANCE}

Each RSU maintains a sample average of the most recent headway distance information. This corresponds to the data currently in the headway distance buffer. We define the Average Headway Distance (AHD) to be the sample mean of the average headway inferred from the data available in the headway distance buffer.

Let us assume that the headway distance buffer contains the following values: 



$$
\begin{gathered}
\because H D_{1}, H D_{2}, H D_{3}, H D_{4}, H D_{5} \\
\therefore A H D=\frac{H D_{1}+H D_{2}+H D_{3}+H D_{4}+H D_{5}}{5}
\end{gathered}
$$

and 1 Unit is

$$
1 U=A H D+L
$$

where $\mathrm{L}$ is the estimated length of a vehicle on the highway

$$
\begin{gathered}
L \epsilon\{\text { Truck, Vehicle }\} \\
L=\frac{T \% * L_{t}+V \% * L_{v}}{T \%+V \%}
\end{gathered}
$$

where $\mathrm{T}$ are Trucks and $\mathrm{V}$ are Vehicles

Assume, we have $30 \%$ Trucks and $70 \%$ Vehicles on the highway

$$
\begin{gathered}
\text { Then } L=\frac{30 \% * L_{t}+70 \% * L_{v}}{0.3+0.7} \\
\text { Then } L=0.30 * L_{t}+0.70 * L_{v}
\end{gathered}
$$

Assume that the length of the vehicle is 5 meters, which is the length of a full size car, and the length of the truck on average 15 meters. Then $L=8$ meters.

\subsubsection{RSU COMMUNICATION WITH THE SCES}

Assume that the RSUs become aware of an incident. The first task that is performed is to locate the exact segment where the incident has occurred. Equivalently, this amounts to identifying an adjacent pair of RSUs that flank the incident on both sides.

However, in order to pinpoint the exact location of the incident and that of the corresponding Head and Tail, it becomes necessary for the RSUs to collect speed data from SCEs. A RSU can request information from SCEs in the surrounding area. SCEs can propagate the request to adjacent SCEs until a response is returned back. This type of communication is done on demand only to save power. Communication from RSU to SCEs is done using broadcast. A RSU can send a broadcast message to SCEs requesting information with the direction required.

\subsection{SCE COMMUNICATION}

Along with the RSUs, the SCEs are the workhorses of FRIEND. The SCEs play the role of having an exact view of the highway all over the segments. Although SCEs work only on demand in case of sudden change of traffic pattern, their main jobs are: 
1. to communicate with vehicles on the highway to collect information about the traffic flow;

2. to identify (on demand) the exact location of an incident or event;

3. to keep track of Head and Tail of an incident as mention in Section 5.3.4.

Although there are three different types of communication involving SCEs. These types of communication can be working simultaneously and even can give a feedback from one to other. Using different types of technology helps in avoiding collisions. However, power consumption is important in SCEs, so most of the communication is done only when requested, as shown in Figure 32, the following three types of communication involving the SCEs are:

1. communication from SCEs to RSUs,

2. communication between adjacent SCEs,

3. communication from vehicles to SCEs.

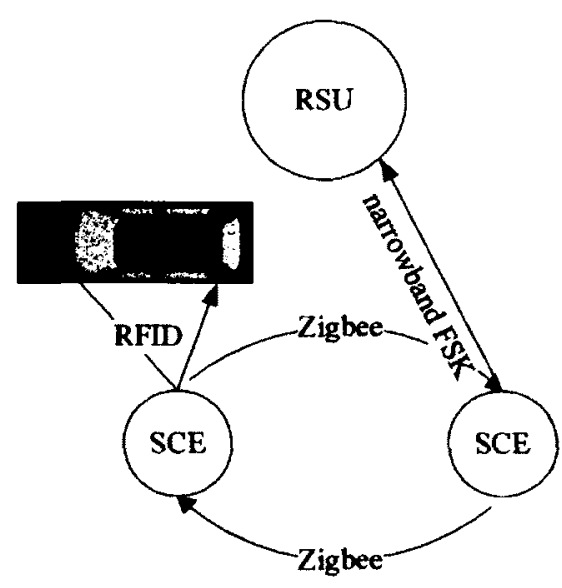

FIG. 32. Three different types of communications for SCEs.

\subsubsection{COMMUNICATION BETWEEN SCES AND RSUS}

The SCEs respond to a request sent from the closest RSU. We use simple narrow-band FSK radio data transmitters that turn on within milliseconds and draw only $10-20 \mathrm{~mA}$. 
Adjacent-channel interference and jamming are very real problems, but can be mitigated by using a frequency-agile narrow-band system. Since this communication does not require a high data rate, we choose to use narrow-band FSK data transceivers in SCEs, as in [111].

Each SCE randomly selects a time slot within a 60-second interval and transmits there. If it detects that another SCE is present when its randomly selected transmission time arrives, it waits for it to pass before transmitting. Redundancy and sparse use of the channel reduce the probability of collisions to an acceptable level.

\begin{tabular}{|c|c|c|c|c|c|}
\hline Packer into & SCE ID & $\begin{array}{c}\text { Information about Vohicles in sogment [I. -11] } \\
\text { (Avorage Speed. Count) }\end{array}$ & SCE ID 2 & Recorded data at SCE ID 2 & Unused spaces \\
\hline
\end{tabular}

FIG. 33. Packet format for data sent from SCE to RSU.

For a sparse time-multiplexed network with $n$ nodes and $s$ time slots, the probability of no collisions during a cycle is

$$
P(\text { nocollisions })=\frac{C(s, n)}{C(n+s-1, n)}
$$

Where $C(s, n)$ is the number of combinations that do not involve a collision and $C(n+$ $s-1, n)$ total number of combinations.

With $\mathrm{n}=10$ per RSU (which means in this case that RSU can communicate with 10 different SCEs), there is a $1.2 \%$ chance of a collision per minute. The chance of a collision occurring during two consecutive 60-second intervals, assuming good random number generators in the nodes is $(1.2 \% * 1.2 \%)=0.014 \%$, so the expected time between any two consecutive collisions is about two hours. However, the expected time between collisions that cause data loss is greater, because the collisions would have to involve the same node [111].

The packet format is shown in Figure 33 [111]. The first field is the packet info, which can be the system type, software version and any other information: Then, comes the node ID, and we assume that the SCE ID will take around 2 bytes which gives 16bits. Next is information about vehicles, including the average speed and the count of vehicles involved, we assume 18 bytes (as in [111]). Then, in case of information sent to another SCE to check the speed and density measured in the middle of the segment, another node 
ID 2 is included with data from SCE 2. Finally, an unused space is reserved for future enhancements (e.g. security).

\subsubsection{COMMUNICATION BETWEEN ADJACENT SCES}

The SCEs are networked together in order to request information about the highway flow traffic condition in middle of a segment or to identify the location of an incident. This type of communication happens only on demand and it is in the case of an incident in the middle segment that cannot have a direct communication with a RSU.

As mentioned before, in case of an incident that occurs on the highway, the first concern is to identify the pair $R S U-R S U[i, i+1]$ of RSUs that flank the segment where the incident occurred. It can be done by asking each RSU what flow it has seen and by propagating the question until identifying the $R S U-R S U[i, i+1]$ where the incident takes place as shown in Section 5.2.

Second, $R S U_{i}$ and $R S U_{i+1}$ send a broadcast request to the SCEs in the nearby area to request the exact segment of incident. When $R S U_{i}$ broadcasts its request, only the SCEs in the direction of traffic will start the process. And when $R S U_{i+1}$ broadcasts its request, only the SCEs in the opposite direction of traffic will start the process as shown in Figure 34.

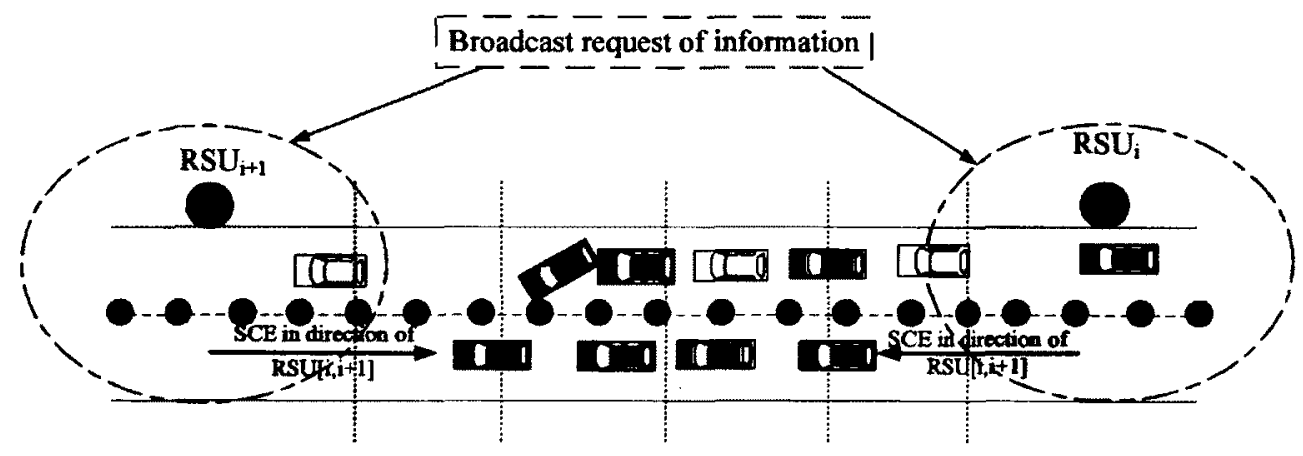

FIG. 34. RSU requesting information from SCEs in the direction of RSU-RSU[i,j].

Third, after sending the request to SCEs, the request is propagated to identify the segment of an incident. Both sides of SCEs propagate the request to the next segment of SCEs as shown in Figure 35. The moment a reply is received, information is sent back to the RSU to inform adjacent RSUs. 


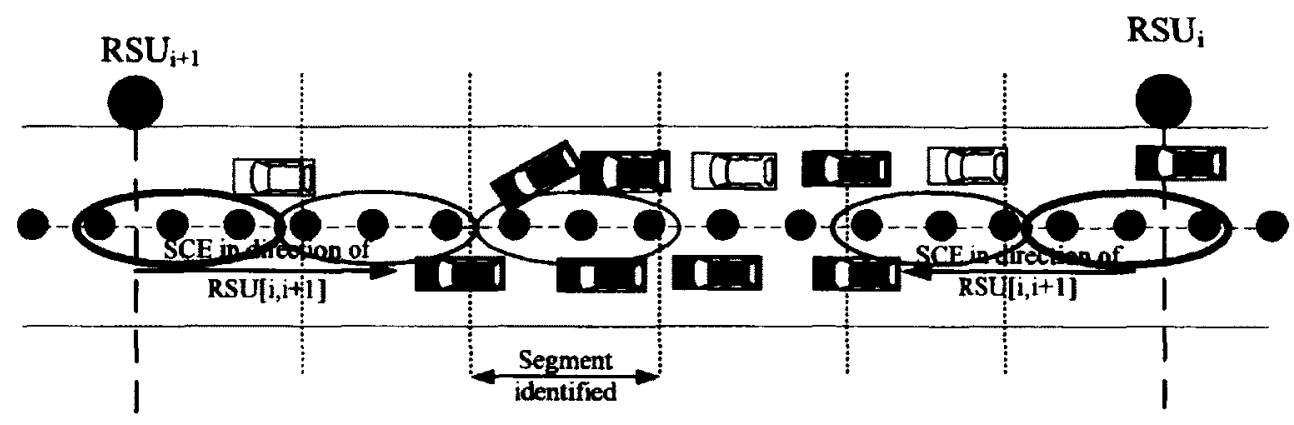

FIG. 35. SCEs identifying the segment of an incident.

We note that the chance that the SCEs need to communication with other SCEs is equal to the chance that the incident or event occurs in the middle of one of the segments. Assuming we have six segments, then the chance is $\frac{1}{3}$.

\subsubsection{COMMUNICATION FROM VEHICLES TO SCES}

FRIEND assumes the use of RFID technology as the communication medium between the smart wheels and SCEs, as mentioned in Chapter 3. The details of the RFID-based communication that takes place between the smart wheels of vehicles and SCEs follows. The RFID reader in the smart wheels allows the vehicle to inform the SCEs about speed, stability loss due to road conditions (if any), and ambient temperature. The SCEs collect data sent from vehicles every $\Delta t$, where $t$ depends on highway conditions. The RFID reader in the smart wheels transmits an object identity using electromagnetic waves. In the SCE, an RFID tag stores its ID in memory. The RFID reader which is installed in the vehicle wheels emits RF radio waves eliciting a signal back from the tag. We use RFID with a radio range up to approximately $3 \mathrm{~m}$. The most important benefit of an RFID tag is the battery-free operation. A tag works without a power source since it gathers energy from a reader's waves [135].

The idea of having a tag on both sides of the SCE is feasible due to the four sides a SCE has, two of them used as reflectors (the same way they have been used for years) and the other two can hold the RFID tag to exchange information with passing vehicles. This idea can be improved later to allow giving information to passing vehicles. Figure 36 shows an exchange of information between a vehicle smart wheel reader and a tag installed in a SCE node. It is important to mention that the term "smart wheels" has been used for a while in vehicle manufactures as the inside steering wheel that has the capability to control many functions in the vehicles. However, our term "smart wheels" refers to the actual wheels of 
the vehicles, and we expect that the RFID reader can be installed in the middle of the rim itself.

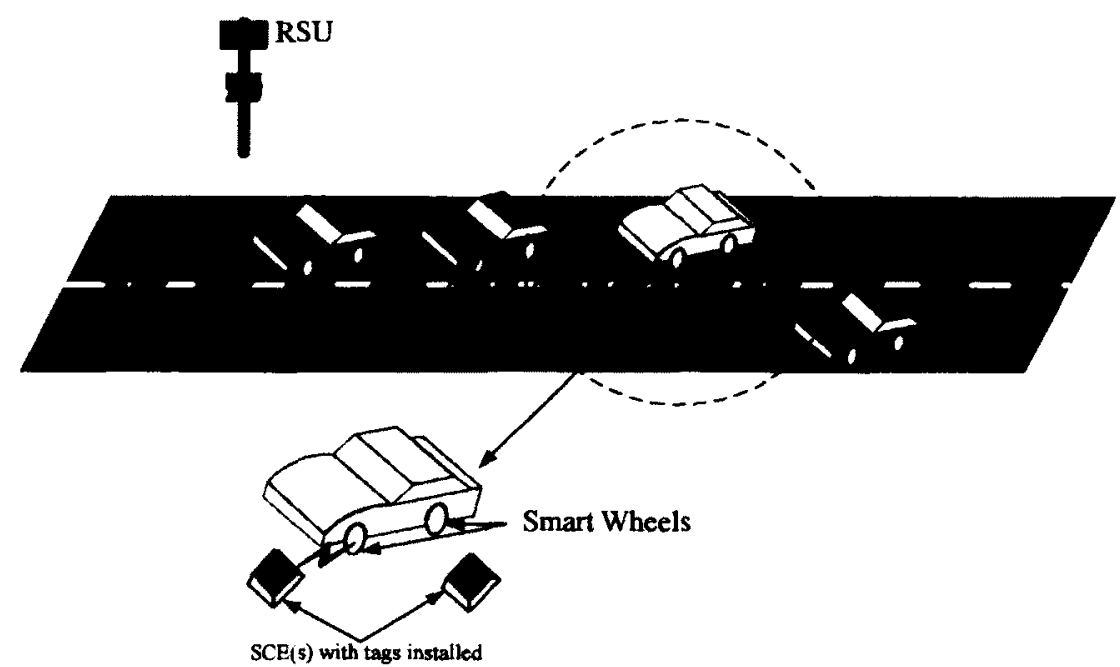

FIG. 36. SCEs exchanging data with smart wheels in vehicles.

\subsubsection{IDENTIFYING THE LOCATION OF HEAD AND TAIL}

In this subsection, we explain how RSUs with help of the SCEs keep track of the traffic backup size. First, we start with some definitions of Head and Tail of an incident or event and what is meant by principal of locality. Then, we explain our technique to track the backup.

Definition 5-3: "Head of a backup" We define the Head of a backup to be the location of the incident. In case an incident has been cleared, it is the start location of the backup where speed of vehicles tend to be almost zero.

Definition 5-4: "Tail of a backup" We define the Tail of a backup to be the end of a backup, where vehicles' speeds decrease rapidly.

Definition 5-5: "Principle of Locality" Let $S_{1}, S_{2}, S_{3}, \ldots S_{i} \ldots, S_{n}$ be a group of consecutive SCE nodes on the highway. The distance between two consecutive nodes on the highway is known as $d$. We define the locality of nodes as recording the same parameters or data over a given distance. Referring to Figure 37, in case of normal 
density traffic or sparse traffic with no incidents, nodes from $S_{1}$ to $S_{j}$ where $j<i$ record the same data, which we call locality of nodes [109].

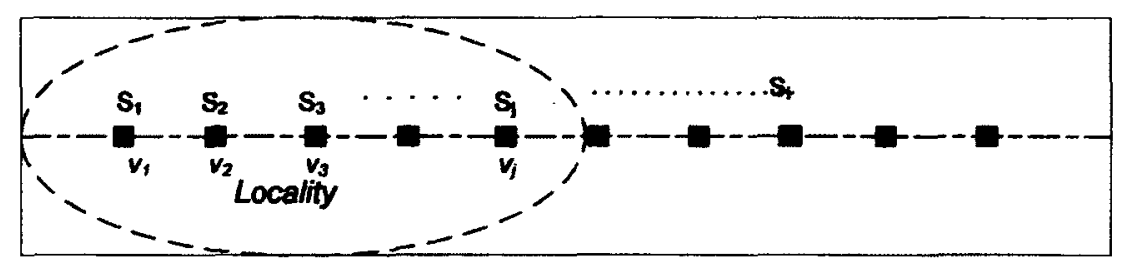

FIG. 37. Illustrating the principle of locality.

To identify the initial Head of the backup is simple; this is the location of the incident until it has been cleared. The moment the Head is identified, a request from $R S U_{i}$ to the previous $R S U_{x}$ where $x<i$, is sent to inform it of the location of the Tail of the backup. After identifying the Tail segment, a SCE request will be generated to identify the exact location. $R S U_{x}$ will reply with both the location of Tail and average speed of previous segment as shown in Figure 38.

\subsubsection{TRACKING THE HEAD AND TAIL OF A TRAFFIC BACKUP}

For simplicity, we assume that only one backup has occurred on the highway. However, the scenario of having more than one backup on the highway due to multiple incidents or events can occur, which will be studied in future work. In Chapter 6, we give more details about data required to track the traffic backup. In brief, to keep track of the Head and Tail locations, FRIEND keeps track of the following parameters:

1. incident location and status;

2. time of the incident and time of the last update;

3. speed of vehicles moving after incident clearance;

4. speed of vehicles reaching the tail of the backup. 


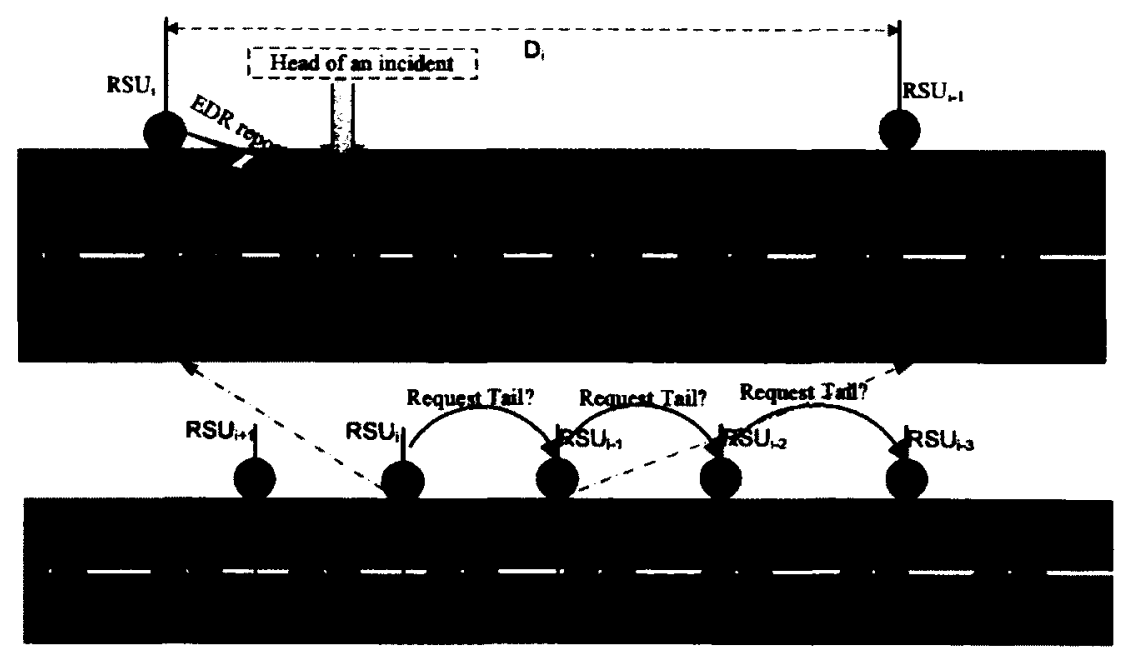

FIG. 38. Identifying the Head and Tail of a backup created from an incident on the highway

\subsection{SUMMARY}

In this chapter, we have addressed the communication protocols employed by each of the entities in FRIEND. We explained how RSUs communicate with adjacent RSUs, vehicles, and SCEs. We also evaluated the average headway distance. The communication protocols for the SCEs with RSUs, adjacent SCEs, and vehicles are described in detail. Finally, we explained the identification of an incident location and how tracking the Head and Tail occur. 



\section{CHAPTER 6}

\section{MAKING TRAFFIC-RELATED DECISIONS IN FRIEND}

As mentioned before, in most of the current VANET-based systems, individual vehicles are responsible for inferring the presence of an incident on the basis of reports received from other vehicles. This invites a host of serious and well-documented security attacks intended to cause vehicles to make incorrect inferences, possibly resulting in increased traffic congestion and a higher chance of severe accidents. On the other hand, it is very hard for most of the existing purely ITS-based Automated Incident Detection (AID) techniques to detect incidents in relatively sparse traffic, especially those incidents that do not block/occlude all lanes.

The past few years have witnessed a rapid converge of ITS and VANET leading to the emergence of Intelligent Vehicular Networks (InVeNet) with the expectation to revolutionize the way we drive by creating a safe, secure, and robust ubiquitous computing environment that will eventually pervade our highways and city streets. As part of the InVeNet partnership, vehicular networks are expected to be instrumental in helping the existing ITS infrastructure with the following tasks:

- informing the driving public and other interested parties about the current status of local traffic;

- AID, especially with those tasks that are either not feasible or, indeed, impossible under current ITS technology;

- traffic-related information dissemination to the driving public and other interested parties.

One of the important contributions of FRIEND is to provide support for detecting traffic incidents and for disseminating traffic-related information to the drivers both in the form of a color-coded instantaneous traffic status report and in the form of more specific incident-related information. The intention is for the color-coded instantaneous traffic status report to be available, as a community service, to all the cars in the traffic, regardless of whether or not they have contributed their underlying EDR data. The color-coded information can be displayed in a suitable form to give the driver an "at-a-glance" synopsis of the state of the traffic up to ten miles ahead. 
The main purpose of this chapter is to present the various mechanisms whereby FRIEND makes decisions about the state of the traffic and also about the possible occurrence of traffic-related incidents.

Recall that FRIEND bases most of the inferences it makes about the status of traffic on two perceived parameters of the traffic flow: speed and density. In turn, the instantaneous density of the flow is deduced by sampling the headway distance of the most recently passing cars. A fundamental theoretical question that we address is the extent to which the sample mean of the collected headway distance data is a good approximation of the overall headway distance. Our results are offered in Section 6.1.

The traffic parameters discussed above along with historical data collected over a reasonable time period (duly adjusted for diurnal and seasonal variations), and with the aggregated traffic information firmly in hand, FRIEND is ready to disseminate to the traveling public a color-coded traffic status report. The details of what is involved can be found in Section 6.2.

Section 6.3 presents the classification of traffic-related incidents employed by FRIEND. Next, Sections 6.4 and 6.5 offer the details of the tasks involved in incident detection and subsequent dissemination of traffic-related information to the driving public and other parties.

\subsection{REASONING ABOUT THE SAMPLE MEAN OF HEADWAY DISTANCE}

It is of great theoretical interest and unmistakable practical relevance to evaluate the suitability of the sample mean of collected headway distances as a bona-fide approximation of the population headway distance.

While this is a difficult, and to the best of our knowledge, unsolved problem under general traffic conditions, we offer a relatively simple answer in the case of free-flow traffic. Such traffic can be thought of as a Poisson process with rate $\lambda>0$. In turn, this assumption implies that the headway distances are exponential random variables with mean $\frac{1}{\lambda}$.

Recall that, as pointed out in Chapter 4, contemporary studies have confirmed that under most traffic conditions the headway distance on highways is best described by a log-normal distribution. It is reassuring to know that, as pointed out by Tijms [165] (see p. 445), the densities of the exponential, log-normal and Weibull distributions have a very similar, indeed almost identical, shape. This mathematical fact explains, to some extent, the reason for the controversy about the distribution of the headway distance in the 
ITS literature. We refer to [4] for a comprehensive discussion of the history of headway distance.

We take advantage of the similarity between the distribution functions of the lognormal and exponential distributions to proceed with the assumption of exponentiallydistributed headway distance, at least in the free-flow regimen.

Assume that a given RSU has collected and recorded $N(t)$ headway distance readings $X_{1}, X_{2}, \cdots, X_{N(t)}$ in the interval $(0, t]$. Assume, further, that the $X_{i} \mathrm{~s},(1 \leq i \leq N(t))$, are independent exponential random variables with mean $\frac{1}{\lambda}$. It is natural to assess how closely the sample mean

$$
\frac{X_{1}+X_{2}+\cdots+X_{N(t)}}{N(t)}
$$

approximates the population mean $\frac{1}{\lambda}$. For this purpose, we propose to evaluate the expression

$$
\frac{1}{\lambda}-E\left[\frac{X_{1}+X_{2}+\cdots+X_{N(t)}}{N(t)}\right]
$$

representing the difference between the population mean and the sample mean. Our results are summarized in the following statement.

Theorem 6.1.1 Assuming $N(t)>0$, it is the case that

$$
\frac{1}{\lambda}-E\left[\frac{X_{1}+X_{2}+\cdots+X_{N(t)}}{N(t)}\right]=\frac{t}{e^{\lambda t}-1} .
$$

Before proceeding with the proof of Theorem 6.1.1, we take note of three technical results that, in addition to being stepping stones towards the proof, are of an independent interest.

Consider an arbitrary probability space $(\Omega, \mathcal{F}, \operatorname{Pr})$ and let $A, B$ and $C_{n},(n \geq 1)$, be events over the probability space such that $\cup_{n \geq 1} C_{n}=\Omega$ and $C_{i} \cap C_{j}=\emptyset$ for all $i \neq j$. Further, let $X$ be a discrete random variable over the same probability space. We assume that the set of jump points of the distribution function $F_{X}$ of $X$ is $J$.

Lemma 6.1.2 The following statement holds

$$
\operatorname{Pr}[B \mid A]=\sum_{n \geq 1} \operatorname{Pr}\left[B \mid A \cap C_{n}\right] \operatorname{Pr}\left[C_{n} \mid A\right] .
$$

Proof. Observing that

$$
\begin{aligned}
B & =B \cap\left(\cup_{n \geq 1} C_{n}\right) \\
& =\cup_{n \geq 1}\left(B \cap C_{n}\right)
\end{aligned}
$$


we can write

$$
\begin{aligned}
\operatorname{Pr}[B \mid A] & =\operatorname{Pr}\left[\cup_{n \geq 1}\left(B \cap C_{n}\right) \mid A\right] \\
& =\sum_{n \geq 1} \operatorname{Pr}\left[B \cap C_{n} \mid A\right] \\
& =\sum_{n \geq 1} \frac{\operatorname{Pr}\left[B \cap C_{n} \cap A\right]}{\operatorname{Pr}[A]} \\
& =\sum_{n \geq 1} \frac{1}{\operatorname{Pr}[A]}\left(\operatorname{Pr}\left[B \mid C_{n} \cap A\right] \operatorname{Pr}\left[C_{n} \cap A\right]\right) \\
& =\sum_{n \geq 1} \frac{1}{\operatorname{Pr}[A]} \operatorname{Pr}\left[B \mid C_{n} \cap A\right] \operatorname{Pr}\left[C_{n} \mid A\right] \operatorname{Pr}[A] \\
& =\sum_{n \geq 1} \operatorname{Pr}\left[B \mid C_{n} \cap A\right] \operatorname{Pr}\left[C_{n} \mid A\right]
\end{aligned}
$$

and the proof of Lemma 6.1.2 is complete.

For the proof of Theorem 6.1.1 we also need the following technical result that provides a closed form for the expected value of $X$ given the event $A$.

Lemma 6.1.3 The following statement holds

$$
E[X \mid A]=\sum_{n \geq 1} E\left[X \mid C_{n} \cap A\right] \operatorname{Pr}\left[C_{n} \mid A\right] .
$$

Proof. Since $X$ is discrete,

$$
\begin{aligned}
E[X \mid A] & =\sum_{x \in J} x \operatorname{Pr}[\{X=x\} \mid A] \\
& =\sum_{x \in J} x \sum_{n \geq 1} \operatorname{Pr}\left[\{X=x\} \mid C_{n} \cap A\right] \operatorname{Pr}\left[C_{n} \mid A\right] \\
& =\sum_{n \geq 1} \operatorname{Pr}\left[C_{n} \mid A\right] \sum_{x \in J} x \operatorname{Pr}\left[\{X=x\} \mid C_{n} \cap A\right] \\
& =\sum_{n \geq 1} \operatorname{Pr}\left[C_{n} \mid A\right] E\left[X \mid C_{n} \cap A\right] \\
& =\sum_{n \geq 1} E\left[X \mid C_{n} \cap A\right] \operatorname{Pr}\left[C_{n} \mid A\right]
\end{aligned}
$$

[by Lemma 6.1.2]

and the proof of Lemma 6.1.3 is complete.

Suppose that $n$ points $T_{1}, T_{2}, \cdots, T_{n}$ are distributed uniformly and independently over $(0, t]$ and let

$$
0=T_{(0)}<T_{(1)}<T_{(2)}<\cdots<T_{(n)}<t
$$


be the corresponding order statistics. We are interested in the distribution of the random variables

$$
T_{(1)}-T_{(0)}, T_{(2)}-T_{(1)}, \cdots, T_{(n)}-T_{(n-1)} .
$$

More specifically, we prove the following result.

Lemma 6.1.4 For all $k=0,1, \cdots, n-1$,

$$
\operatorname{Pr}\left[\left\{T_{(k+1)}-T_{(k)} \leq y\right\}\right]= \begin{cases}0 & \text { for } y \leq 0 \\ 1-\left(1-\frac{y}{t}\right)^{n} & \text { for } 0<y \leq t \\ 1 & \text { for } y>t\end{cases}
$$

Proof. A possible proof goes as follows; let $k$ be arbitrary but fixed and $0 \leq y \leq t$ Assume without loss of generality that $T_{(k)}=x$; observe that conditional on $\left\{T_{(k)}=x\right\}$, the event $\left\{T_{(k+1)}-T_{(k)} \leq y\right\}$ occurs if and only if $k-1$ points lie in $(0, x)$, one point lies in some differential interval $[x, x+\mathrm{d} x)$ and the remaining $n-k$ points lie in the interval $(x+y, t]$

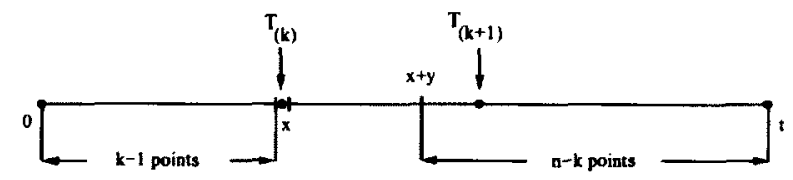

FIG. 39. Illustrating the proof of Lemma 6.1.4.

Notice that for a given $x$ the probability that:

- the probability that exactly one point lies in $[x, x+\mathrm{d} x)$ is $\left(\begin{array}{l}n \\ 1\end{array}\right) \frac{\mathrm{d} x}{t}$,

- the probability that of the remaining $n-1$ points, $k-1$ lie in $(0, x)$ is $\left(\begin{array}{c}n-1 \\ k-1\end{array}\right)\left(\frac{x}{t}\right)^{k-1}$.

Thus, for a given $x$ the conditional probability that the event $\left\{T_{(k+1)}-T_{(k)} \leq y\right\}$ occurs given that $\left\{T_{(k)}=x\right\}$ is

$$
n\left(\begin{array}{l}
n-1 \\
k-1
\end{array}\right)\left(\frac{x}{t}\right)^{k-1}\left(\frac{t-x-y}{t}\right)^{n-k} \frac{\mathrm{d} x}{t}
$$


and so the probability $P_{k}$ of the event $\left\{T_{(k+1)}-T_{(k)} \leq y\right\}$ is

$$
P_{k}=\int_{0}^{t-y} n\left(\begin{array}{l}
n-1 \\
k-1
\end{array}\right)\left(\frac{x}{t}\right)^{k-1}\left(1-\frac{x+y}{t}\right)^{n-k} \frac{\mathrm{d} x}{t}
$$

All that remains is to evaluate

$$
P_{k}=\int_{0}^{t-y} n\left(\begin{array}{l}
n-1 \\
k-1
\end{array}\right)\left(\frac{x}{t}\right)^{k-1}\left(1-\frac{x+y}{t}\right)^{n-k} \frac{\mathrm{d} x}{t}
$$

As it turns out, this integral reduces to the Euler's Beta function (see (61) in the Apendix). Indeed, we write

$$
\begin{aligned}
P_{k}= & n\left(\begin{array}{l}
n-1 \\
k-1
\end{array}\right) \int_{0}^{t-y}\left(\frac{x}{t}\right)^{k-1}\left(1-\frac{x+y}{t}\right)^{n-k} \frac{\mathrm{d} x}{t} \\
= & n\left(\begin{array}{l}
n-1 \\
k-1
\end{array}\right) \int_{0}^{1} u^{k-1}(1-u)^{n-k}\left(1-\frac{y}{t}\right)^{n} \mathrm{~d} u \quad\left[\text { after } u=\frac{x}{t-y}\right] \\
= & n\left(\begin{array}{l}
n-1 \\
k-1
\end{array}\right)\left(1-\frac{y}{t}\right)^{n} \int_{0}^{1} u^{k-1}(1-u)^{n-k} \mathrm{~d} u \\
= & n\left(\begin{array}{l}
n-1 \\
k-1
\end{array}\right) \operatorname{Beta}(\mathbf{k}, \mathrm{n}-\mathbf{k}+1)\left(1-\frac{\mathrm{y}}{\mathrm{t}}\right)^{\mathrm{n}} \\
= & \frac{n !}{(k-1) !(n-k) !} \frac{\Gamma(k) \Gamma(n-k+1)}{\Gamma(n+1)}\left(1-\frac{y}{t}\right)^{n} \\
& {[\text { by Theorem 62 in the Appendix] }} \\
= & \frac{n !}{(k-1) !(n-k) !} \frac{(k-1) !(n-k) !}{n !}\left(1-\frac{y}{t}\right)^{n} \\
& {[\text { by Theorem 60 in the Appendix] }} \\
= & \left(1-\frac{y}{t}\right)^{n},
\end{aligned}
$$

independent of $k . \square$

Lemma 6.1.4 has two important corollaries that we state and prove next.

Corollary 6.1.5 For all $k=0,1, \cdots, n-1$,

$$
E\left[T_{(k+1)}-T_{(k)}\right]=\frac{t}{n+1}
$$

Proof. Letting $G$ stand for the probability distribution function of the random variable $T_{(k+1)}-T_{(k)}$, it is well known that

$$
E\left[T_{(k+1)}-T_{(k)}\right]=\int_{0}^{\infty}[1-G(y)] \mathrm{d} y .
$$


With this, we can write

$$
\begin{aligned}
E\left[T_{(k+1)}-T_{(k)}\right] & =\int_{0}^{\infty}[1-G(y)] \mathrm{d} y \\
& =\int_{0}^{t}[1-G(y)] \mathrm{d} y \\
& =\int_{0}^{t}\left(1-\frac{y}{t}\right)^{n} \mathrm{~d} y \\
& =\int_{0}^{1} t u^{n} \mathrm{~d} u \quad\left[\operatorname{after} u=1-\frac{y}{t}\right] \\
& =\frac{t}{n+1}
\end{aligned}
$$

and the proof of Corollary 6.1 .5 is complete.

In turn, Corollary 6.1.5 implies the following result

Corollary 6.1.6 For all $k=0,1, \cdots, n$

$$
E\left[T_{(k)}\right]=\frac{k t}{n+1}
$$

Proof. The proof is by induction on $k$. To settle the basis, observe that since $T_{(0)}=0$, Corollary 6.1.5 implies that

$$
E\left[T_{(1)}-T_{(0)}\right]=E\left[T_{(1)}\right]=\frac{t}{n+1} .
$$

Let $k$ be arbitrary and assume that $E\left[T_{(k)}\right]=\frac{k t}{n+1}$. We need to show that

$$
E\left[T_{(k+1)}\right]=\frac{(k+1) t}{n+1}
$$

The linearity of expectation yields

$$
\begin{aligned}
\frac{t}{n+1} & =E\left[T_{(k+1)}-T_{(k)}\right] \\
& =E\left[T_{(k+1)}\right]-E\left[T_{(k)}\right] \\
& =E\left[T_{(k+1)}\right]-\frac{k t}{n+1}
\end{aligned}
$$

which, in turn, implies (42) and the proof is complete.

Proof of Theorem 6.1.1 Recall that we assumed $N(t)>0$. It should be clear that the event $\{N(t)>0\}$ is equivalent to $\cup_{n \geq 1}\{N(t)=n\}$ and that the events $\{N(t)=i\}$ and $\{N(t)=j\}$ are disjoint whenever $i \neq j$. It follows that for all $n \geq 1$,

$$
\{N(t)>0\} \cap\{N(t)=n\}=\{N(t)=n\} .
$$


Now, Lemma 6.1.3 allows us to write

$$
\begin{aligned}
& E\left[\frac{X_{1}+X_{2}+\cdots+X_{N(t)}}{N(t)} \mid\{N(t)>0\}\right] \\
= & \sum_{n \geq 1} E\left[\frac{X_{1}+X_{2}+\cdots+X_{N(t)}}{N(t)} \mid\{N(t)>0\} \cap\{N(t)=n\}\right] \\
& \operatorname{Pr}[\{N(t)=n\} \mid\{N(t)>0\}] \\
= & \sum_{n \geq 1} E\left[\frac{X_{1}+X_{2}+\cdots+X_{N(t)}}{N(t)} \mid\{N(t)=n\}\right] \\
& \operatorname{Pr}[\{N(t)=n\} \mid\{N(t)>0\}] . \text { [by (44)] }
\end{aligned}
$$

We now proceed to evaluate the various ingredients of (45). To begin,

$$
\begin{aligned}
\operatorname{Pr}[\{N(t)=n\} \mid\{N(t)>0\}] & =\frac{\operatorname{Pr}[\{N(t)=n\} \cap\{N(t)>0\}]}{\operatorname{Pr}[\{N(t)>0\}]} \\
& =\frac{\operatorname{Pr}[\{N(t)=n\}}{\operatorname{Pr}[\{N(t)>0\}]} \\
& =\frac{\frac{(\lambda t)^{n}}{n !} e^{-\lambda t}}{1-e^{-\lambda t}} \\
& =\frac{(\lambda t)^{n} e^{-\lambda t}}{n !\left(1-e^{-\lambda t}\right)}
\end{aligned}
$$

Next, it is well known that, conditional on $n$ Poisson arrivals in $(0, t]$, the actual times $T_{1}, T_{2}, \cdots, T_{n}$ of these arrivals are uniformly distributed in $(0, t]$. This observation allows us to write

$$
\begin{aligned}
& E\left[\frac{X_{1}+X_{2}+\cdots+X_{N(t)}}{N(t)} \mid\{N(t)=n\}\right] \\
= & E\left[\frac{X_{1}+X_{2}+\cdots+X_{n}}{n} \mid\{N(t)=n\}\right] \\
= & \frac{1}{n} E\left[X_{1}+X_{2}+\cdots+X_{n} \mid\{N(t)=n\}\right] \\
= & \frac{1}{n} E\left[\max \left\{T_{1}, T_{2}, \cdots, T_{n}\right\}\right] \\
= & \frac{1}{n} \frac{t n}{n+1} \text { [by Corollary 6.1.6] } \\
= & \frac{t}{n+1} .
\end{aligned}
$$


Finally, replacing (48) and (49) in (45) yields

$$
\begin{aligned}
& E\left[\frac{X_{1}+X_{2}+\cdots+X_{N(t)}}{N(t)} \mid\{N(t)>0\}\right] \\
= & \sum_{n \geq 1} \frac{t}{n+1} \frac{(\lambda t)^{n} e^{-\lambda t}}{n !\left(1-e^{-\lambda t}\right)} \\
= & \frac{e^{-\lambda t}}{\lambda\left(1-e^{-\lambda t}\right)} \sum_{n \geq 1} \frac{(\lambda t)^{n+1}}{(n+1) !} \\
= & \frac{e^{-\lambda t}}{\lambda\left(1-e^{-\lambda t}\right)}\left[e^{\lambda t}-1-\lambda t\right] \\
= & \frac{1-e^{-\lambda t}-\lambda t e^{-\lambda t}}{\lambda\left(1-e^{-\lambda t}\right)} \\
= & \frac{1}{\lambda}-\frac{t e^{-\lambda t}}{1-e^{-\lambda t}} \\
= & \frac{1}{\lambda}-\frac{t}{e^{\lambda t}\left(1-e^{-\lambda t}\right)} \\
= & \frac{1}{\lambda}-\frac{t}{e^{\lambda t}-1}
\end{aligned}
$$

and the proof of Theorem 6.1.1 is complete.

A few observations are in order at this point.

- First, it is easy elementary to verify that

$$
\lim _{t \rightarrow \infty} \frac{t}{e^{\lambda t}-1}=0
$$

confirming that the larger the sample of headway distances collected by the RSU, the better the approximation of the population mean $\frac{1}{\lambda}$. In common practice, there is a tradeoff between the accuracy of the approximation and the timely detection of any departure from the "normal" headway distance accumulated over time (historic data). One good compromise seems to be to collect headway distance data until it either fills a buffer or else it times out, whichever comes first;

- On the numerical side, Theorem 6.1.1 implies that for $\lambda=1$, i.e. assuming one vehicle passes the RSU per unit time, a sample size of five yields a sample mean that differs from the population mean by 0.03 which, in the interest of expediency, is considered acceptable. Naturally, a larger sample reduces the difference even further, in fact, as close to zero as desired; 
- A final observation, mostly for the cognoscenti, is that the result of Theorem 6.1.1 together with (50) are a confirmation of the Strong Law of Large Numbers. The value added offered by Theorem 6.1.1 is the exact value of the difference between the sample mean and the population mean as a function of $t$. As seen above, this allows to tailor the sampling regimen to suit pre-established QoS parameters in terms of a desired trade-off between latency and accuracy.

\subsection{COLOR-CODED TRAFFIC STATE}

The concept of Level of Service (LoS) is a measure used in ITS by traffic engineers to assess the effectiveness of various elements of transportation infrastructure. LoS is most commonly used to analyze highways, but the concept has also been applied to intersections, transit, and water supply. LoS classifies the state of the traffic, heuristically, into six categories, from $\mathrm{A}$ to $\mathrm{F}$ as follows [166].

- $A=$ Free flow;

- $B=$ Reasonably free flow;

- $\mathrm{C}=$ Stable flow;

- $D=$ Approaching unstable flow;

- $\mathbf{E}=$ Unstable flow;

- $F=$ Forced or breakdown flow.

In order to use the LoS concept, FRIEND maps the various LoS categories into headway distance values. We now describe the detailed mapping between the LoS categories for traffic on highway and a proposed set of colors on the driver monitor. One of the services offered by FRIEND to the traveling public is to monitor the traffic flow on the highways and to provide a colored map system in front of the driver screen. In order to explain the data collection, data processing and information update we take an example of an application from the highest level application which is "Real-time Traffic Monitoring".

Input: Data collected from highway infrastructure and vehicles passing RSUs.

Output: A user friendly touch screen that allows two levels of traffic view, as illustrated in Figure 40. The first level is giving the overall status of the road segment between two adjacent RSUs along the highway. Upon demand, FRIEND can present a more detailed 
view of a given road segment to a finer granularity. In Figure 40 a granularity of 0.25 miles $(\approx 400 \mathrm{~m})$ is shown. The meaning of the various colors is described next.

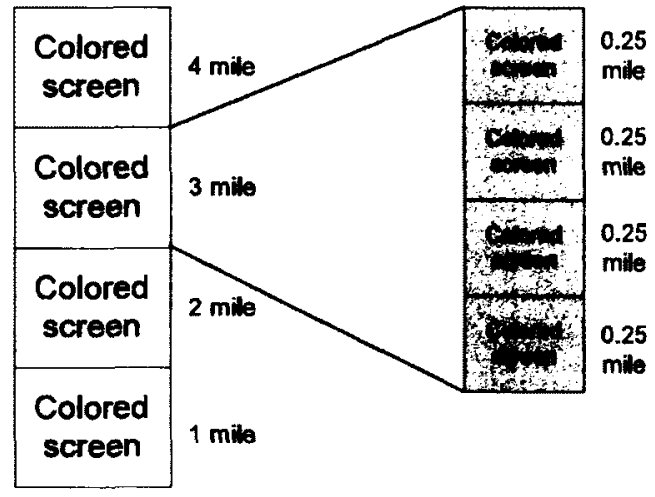

FIG. 40. Illustrating a two-level color-coded display of the state of traffic in FRIEND.

FRIEND maps the current LoS measurement to a four-state Markov chain, each state corresponding to a color, as shown in Figure 41:

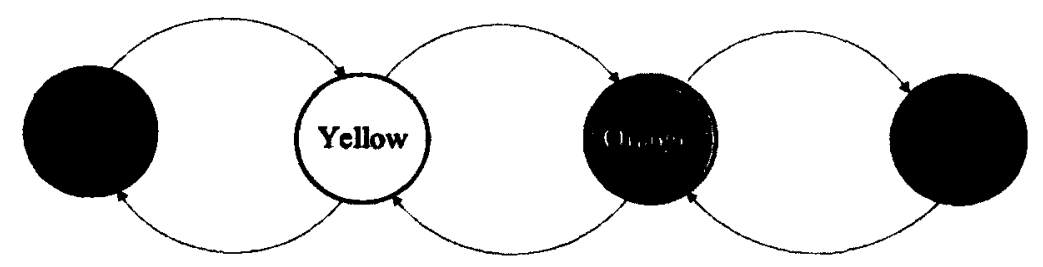

FIG. 41. Illustrating the high-level four-state Markov chain defining color transitions in FRIEND.

The mapping from the LoS letters to the states of the Markov chain is described next.

- Green: an initial state that describes a flow that allows the driver to reach maximum speed on the highway in conjunction with low density. A highway with a green state means that a driver can change lanes easily and can reach maximum speed if such a speed is desired. The green state represents the A and B states in LoS;

- Yellow: a state that describes a flow where traffic is stable but the density is high. A highway with a yellow state means that it is hard to change lanes and speed can be 
below maximum speed for some periods. The yellow state represents the $\mathrm{C}$ state in LoS;

- Orange: a state that describes a speed less than the maximum speed with high density. A highway with orange state means it is approaching an unstable flow and might turn to an unstable flow. The orange state represents the D and $E$ states in LoS;

- Red: a state that describes a breakdown flow where speed is less than $25 \mathrm{miles} / \mathrm{hr}$ and density is almost jammed. A driver seeing a highway with a red state might take an alternate route if possible. The red state represents the F state in LoS.

In order to avoid spurious transitions between colors, FRIEND has a built-in "laziness" that records traffic flow trends without necessarily taking immediate action, i.e. without triggering a state change. FRIEND implements this idea by mapping the high-level fourstate Markov chain discussed above to another, internal Markov chain that keeps more detailed information about the trends in traffic flow. In the internal Markov chain, each state of the high-level Markov chain (i.e. each of the four colors disseminated to the public) is mapped to a set of states of the internal Markov chain as we are about to describe.

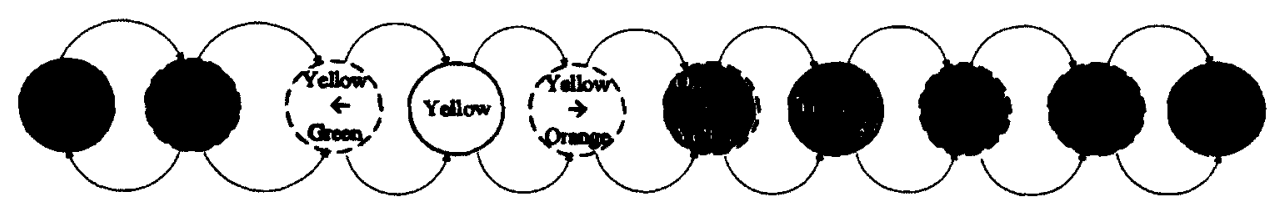

FIG. 42. Illustrating the internal 10-state Markov chain defining color transitions.

Referring to Figure 42, FRIEND maintains an internal 10-state Markov chain with two states for green and red and three states for each of yellow and orange. However, the status report disseminated to the public is just the color without any mention to the specific "shade" of color that the internal Markov chain is in. For example, the reported status is yellow if the internal Markov chain is in any of the three yellow states in Figure 42 . The exact decisions that trigger the actual transitions between states in the internal 10-state Markov chain will be discussed in Subsection 6.2.1.

\subsubsection{TRANSITIONS IN THE INTERNAL MARKOV CHAIN}


The main goal of this subsection is to give the details of the algorithm that induces transitions in the internal Markov chain. The key decision elements that FRIEND employs to effect state transitions in the internal Markov chain are the Average Headway Distance (AHD) and speed (V) aggregated from the most recent EDR data collected from passing cars, along with historical data collected over a longer time span involving months (or even years) of monitoring data at the same locale.

We begin by presenting the mapping algorithm that converts the AHD and speed to colors in Figure 43. Speed is the average speed of vehicles, and MaxSpeed is the highest speed observed from the historical data for the particular time of day and day of week. The safe distance can vary depending on number of lanes, historical data, and maximum speed allowed on the highway as explained in Section 4.2.4. In Figure 45, the transitions in the internal Markov Chain is shown.

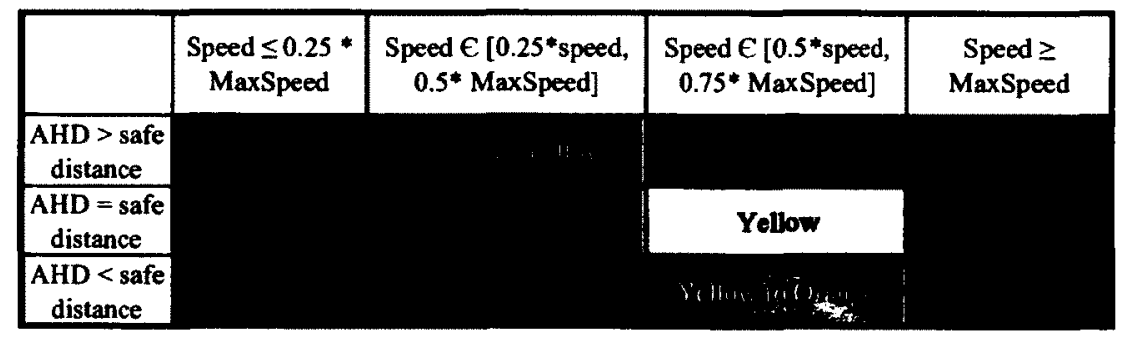

FIG. 43. State diagram and relation with average headway distance and speed.

function Map-State $\left(A H D_{i}, V_{i}\right)$

1. Define SD Safe Distance, MaxV Maximum Speed

2. Define Threshold From Historical Data with time and day input

3. $\mathrm{HD}-\mathrm{Diff}=\left|A H D_{i}-S D\right|$

4. state= lookup-table(HD-Diff, $\left.V_{i}\right)$ As shown in figure 44, represented in 4 bits

5. return (state)

6. end 


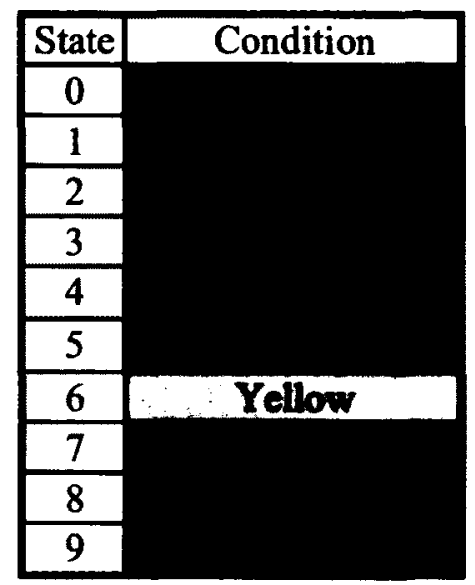

FIG. 44. Truth table for states.

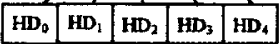

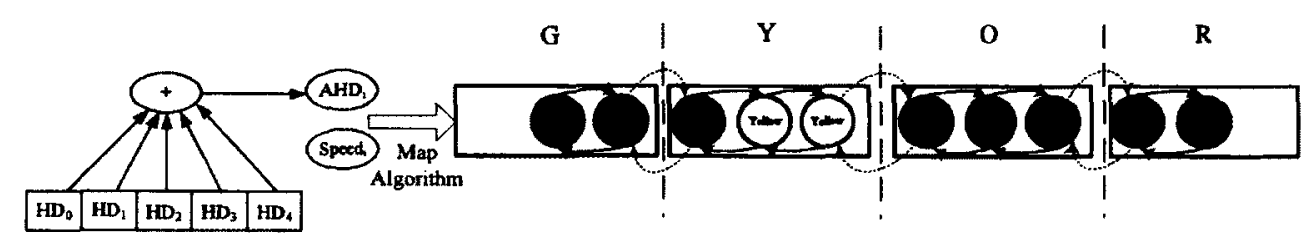

Headway Distance Buffer $i$

FIG. 45. Transitions in the internal Markov chain.

\subsection{INCIDENT CLASSIFICATION}

In this section, we present the classification of incidents used in FRIEND. An incident is defined as any non-recurring event that causes a reduction of highway capacity or an abnormal increase in demand. Such events include traffic crashes, disabled vehicles, spilled cargo, highway maintenance and reconstruction projects, and special nonemergency events (e.g., ball games, concerts, or any other event that significantly affects roadway operations) [167]. In addition weather conditions are often considered incidents that reduce the flow on highways.

FRIEND classifies incidents into three categories: 


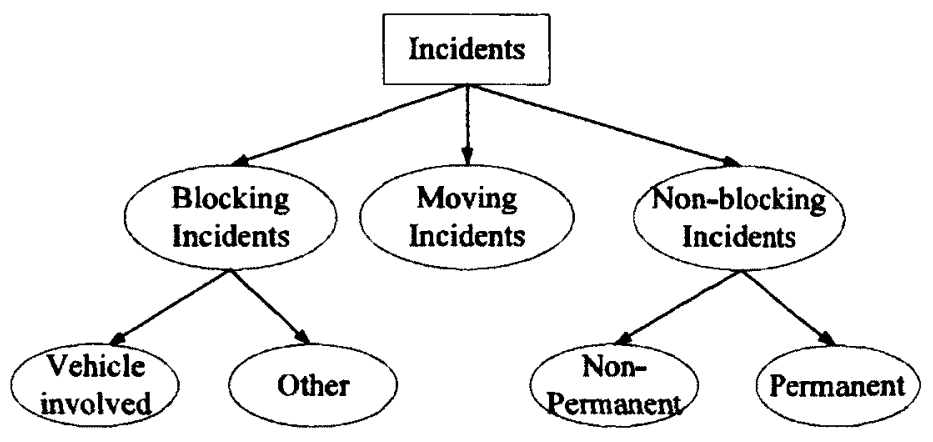

FIG. 46. Highway incident classification.

- First, a blocking incident is an incident that blocks a lane or multiple lanes on highways. As such, an incident blocking one lane of a three-lane freeway reduces capacity by almost 50 percent, although only a third of the lanes are blocked [167];

- Second, a non-blocking incident is an incident that some vehicles can pass over and other will switch lanes if possible. Examples of this kind of incidents include dead animals on the roadway, potholes, lost cargo, and some weather-induced occlusions (such as icy road conditions or accumulated water after a heavy rain or flood). The major difference between a blocking incident and a non-blocking one is that the former renders the lane/road impassable, while the latter does not. In fact, some drivers do not mind driving through a pothole or even standing water;

- Third, a moving incident is a slow-moving vehicle or a convoy of trucks (e.g., military units) traveling together as a group, usually at low speed. This type of incident can affect the flow on the highway and, depending on traffic density, not all drivers will be able to change lanes to pass the convoy.

Figure 46 illustrates the classifications of incidents on highways.

\subsubsection{BLOCKING INCIDENTS}

Blocking incidents are events that cause the occlusion or blocking of one or multiple lanes on the highway. These type of incidents affect the traffic flow and cause a delay in travel time for vehicles. A blocking incident can involve a vehicle or there may be no vehicle involved (i.e. a fallen tree blocking one or more lanes of a highway);

\subsubsection{NON-BLOCKING INCIDENTS}


Non-blocking incidents are non-occluding incidents that some vehicles can pass over and others will have to switch lanes to avoid. We further partition this type of incident into two sub-categories. A non-blocking incident is non-permanent when it occurs for some time after which it disappears. An example of non-blocking non-permanent incidents are icy road conditions in the early morning. The second category represents permanent nonblocking incidents, such as potholes which stay for long time period before being fixed.

\subsubsection{MOVING INCIDENTS}

Moving incidents are non-recurring events that cause a reduction of highway capacity. Referring to Figure 47, a slow-moving truck carrying an over-sized load (e.g. a mobile home) is a typical example of what FRIEND calls a moving incident. Observe that in addition to being slow-moving, the truck turns out to impact the flow of traffic in adjacent lanes. Most of the time, moving incidents are avoided by lane changes. In free traffic flow where vehicles change lanes freely, the effect of a moving incident is often negligible. However, as traffic density increases, the effect of a moving incident becomes more and more pronounced. A driver would like to know if a moving incident will appear, in order to be prepared to change lanes.

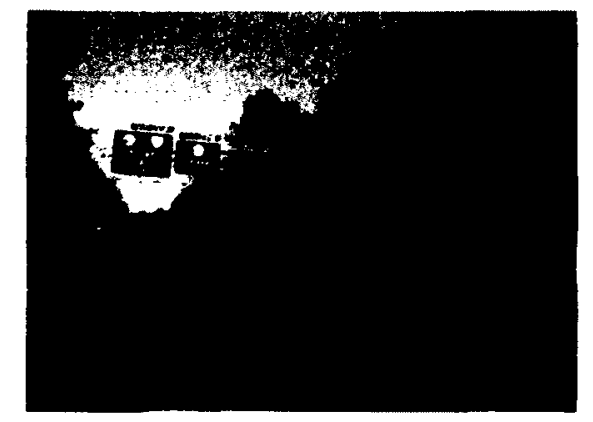

FIG. 47. Example of a moving incident on Interstate 64, Feb 2012

\subsection{INCIDENT DETECTION}

The workhorses of incident detection are the RSUs and the SCEs working together. We defined the term RSU-RSU[i,j] and Segment in Chapter 5 as shown in Figure 28. Recall that the RSUs receive (from most cars) EDR data reporting, among others, any lane change 
that occurred in the current road segment. One of the key ideas of incident detection in FRIEND is that, in case of an incident, there will be numerous correlated lane changes. These transactions will be correlated both in time and space. In this regard, FRIEND builds on the work of Abuelela [168] who has studied how far ahead of an incident drivers will change lanes. Finally, the hardest to detect are moving incidents, which require an intelligent tracking of the offset change of lanes.

Here are, in outline, the tasks performed by the incident detection and information dissemination algorithm for detecting various incidents:

1. Task 0: $R S U$ initialization: Initially, we assume that $R S U_{i}$ has just started to collect data. FRIEND assumes that the RSU initialization involves obtaining the historical data of the highway flow, speed, density and headway distance expected at a specific time or date. Each RSU keeps track of the average speed $v_{a v g}$, headway, and density of vehicles $k_{\text {avg }}$ in the previous $R S U-R S U[i, i-1]$;

2. Task 1: Incident detection: $R S U_{i}$ is notified of an incident or $R S U_{i}$ notices a change of speed or density in RSU-RSU[i,j]. A notification of lane changing in the same location in the previous RSU-RSU[i-1,j] area in a short time, identifies the possibility of an incident. Threshold, which we will call it $T_{h i}$, can be determined from historical data, the higher the threshold, the more time needed to detect an incident and the less chance to generate alarms;

3. Task 1-1: Identifying RSU-RSU: Determining which RSU-RSU[i,j] area where an incident occurs "Global view"; a communication between adjacent RSUs is required to identify the RSU-RSU[i,j] where the incident has occurred;

4. Task 1-2: Identifying segment and location: Identify segment with incident; vehicles that have changed lanes in the last segment report lane change $L_{c}$ and location of lane change (offset). This task can be done using communication between RSU(s) and $\mathrm{SCE}(\mathrm{s})$ as described in Chapter 5;

5. Task 1-3: Classifying the incident: To distinguish between blocking, non-blocking and moving incidents. Figure 48 shows the difference between expected patterns of changing lanes in case of blocking and non-blocking. Figure 49 shows the pattern of moving a incident on highway;

6. Task 2: Information dissemination: To inform other vehicles of an incident or change of highway traffic conditions as explained in Section 6.5. 


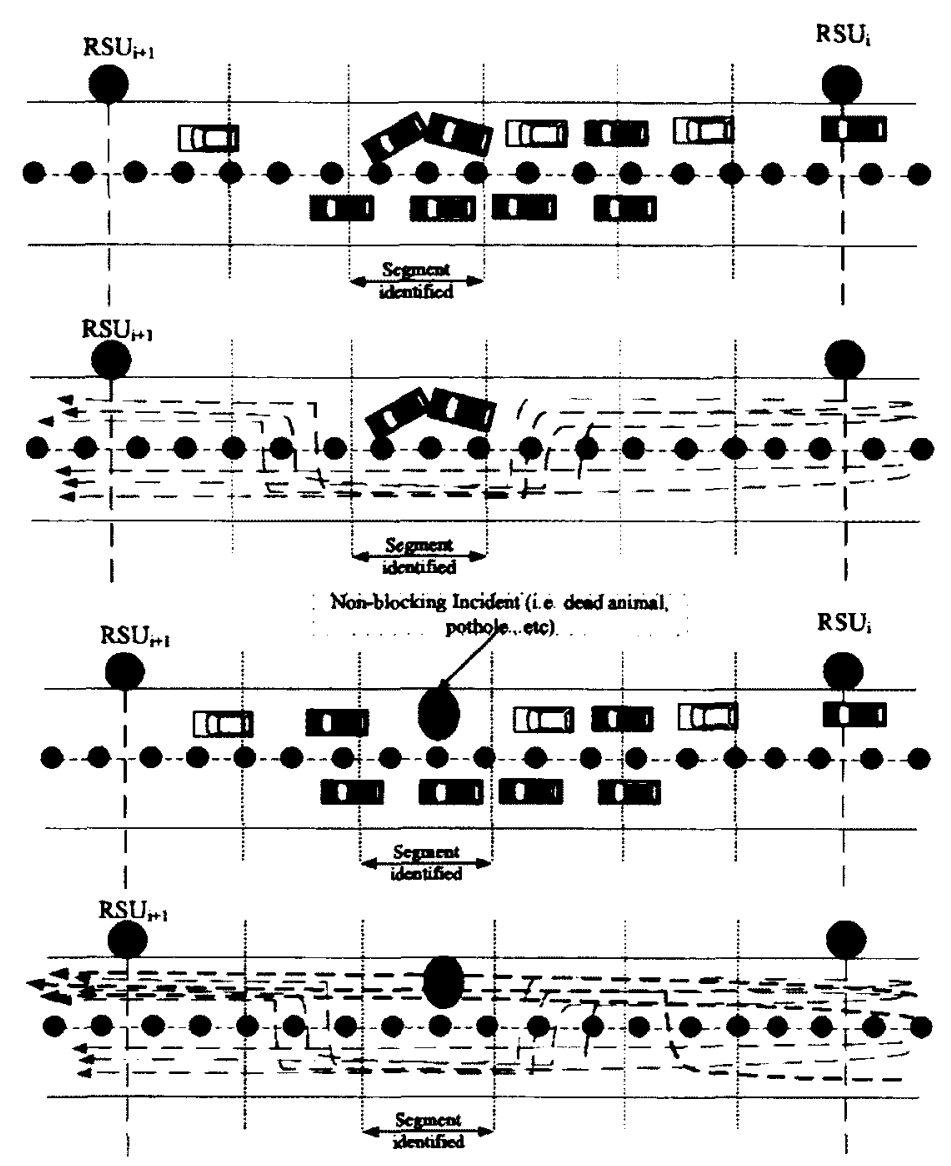

FIG. 48. Different patterns of traffic in case of a blocking and non-blocking incident on highway. Blue lines represent the drivers who have changed lane. Black lines represent the drivers who passed on the pot-hole.

In the case of a moving incident, as shown in Figure 49, the pattern is harder to detect and it requires extra information. We plan to address the issues involved in dealing with moving incidents in future work as described in Chapter 8.

\subsection{INCIDENT INFORMATION DISSEMINATION}

One of the important issues in vehicular networks is the dissemination of information captured from an event or incident to the drivers potentially affected by the incident. In this section, we introduce an information propagation technique that notifies drivers about an incident that has happened along the road. After detecting an incident, as discussed in 


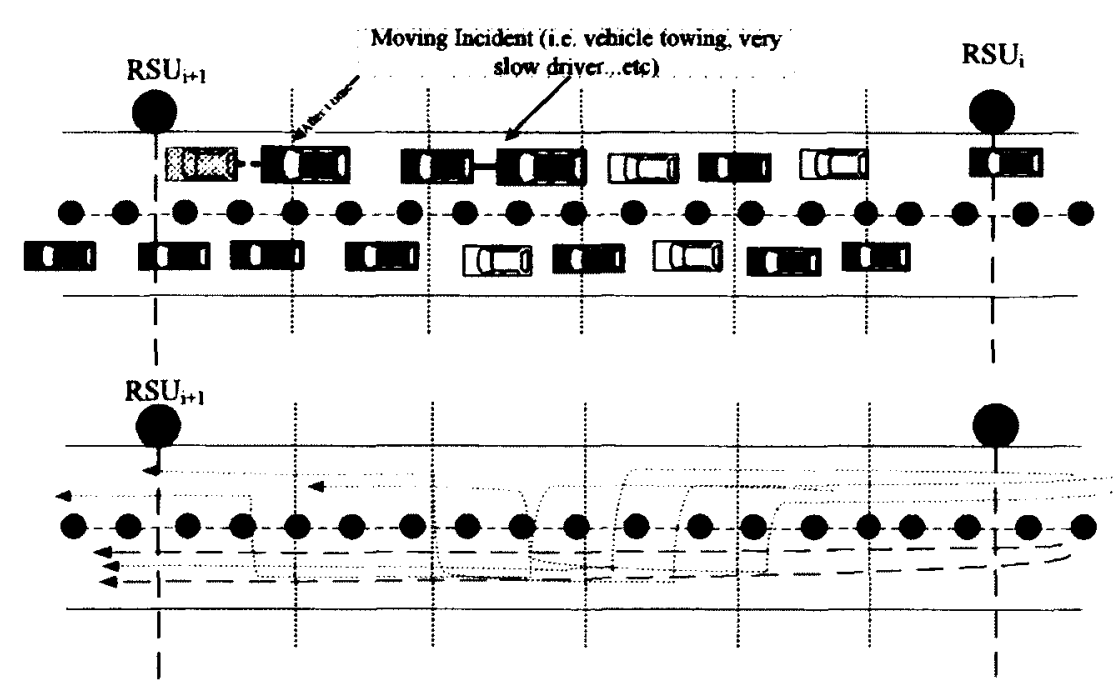

FIG. 49. Patterns of traffic in case of a moving incident on a highway. The change of lane occurs with a shifted distance.

Section 6.4, it is very important to inform vehicles of what a driver should expect to see in the coming miles.

Different types of events or incidents require different levels of propagation depending on how critical the incident and how long it stays. Drivers would like to receive information that affects their decision rather than just notification about incidents that will be solved by the time they reach this point on the highway. Currently GPS with life traffic information can give warning messages about incidents that are far away from other vehicles. Moreover, it depends more on the traffic flow than the event itself. Also, Virginia 511 offered by Virginia Department of Transportation (VDOT) is a similar example of a service that disseminates information on a website or mobile application. Interested drivers can check Virginia 511 for incidents and current highway conditions. A comparison between FRIEND and Virginia 511 is given in Chapter 8.

In FRIEND, the longer the incident stays, the farther the information will be propagated. FRIEND compares different densities with the level or distance of propagation bearing in mind the principle of locality, where drivers very close to an incident can see the incident and close nodes can sense the slowing of the flow on the highway.

In this section, we assume that an incident has just started at time $t_{0}$, and it is detected using the technique in Section 6.4. We have two aims for information propagation. First, we aim to prevent secondary accidents $[98,169]$. A secondary accident is an accident that 
occurs after another accident. Second, we notify drivers far away from the accident of an expected delay by updating their coloring system. FRIEND gives the drivers the option to continue or exit before reaching the location of the incident. RSUs can disseminate the information backwards to the other nodes on the roadway. Most current research focuses on one of the two aims mentioned above. Wisitpongphan et al. [99] depend on vehicles to forward the messages, which can suffer from disconnection problems with sparse (few number of vehicles) traffic. Moreover, they cannot solve the problem of blocking incidents, for example a vehicle that blocks the road completely.

\subsubsection{THE PROPOSED TECHNIQUE}

Our idea of information propagation depends on two main factors: density of the traffic and time necessary to clear the accident. The longer the incident stays, the farther the information needs to be propagated. Furthermore, the higher the density on the highway, the faster a backup will build. In high density highways, an incident may form a backup of vehicles faster than on low density highways. In order to satisfy this point, we need to propagate information as a function of density and time.

In FRIEND the dissemination is handled in two stages:

- Stage I: Focus on the first goal, which is notifying vehicles close to an accident;

- Stage II: A new mechanism to track the source of the incident, while notifying drivers away from the accident, as shown in Figure 50.

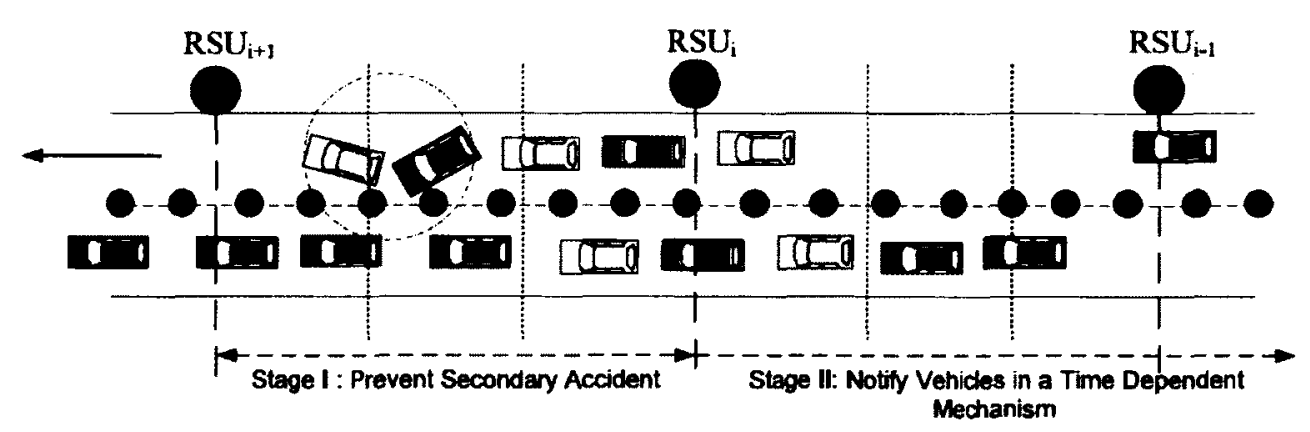

FIG. 50. The two stages of incident dissemination in FRIEND.

In Stage I, the RSU is responsible for informing the previous RSU immediately of the incident to the vehicles passing beside it of the incident. The longer the incident takes to 
be cleared, the more frequently the previous RSU will be informed of the incident. Figure 51 shows how Stage I works in case of an incident.

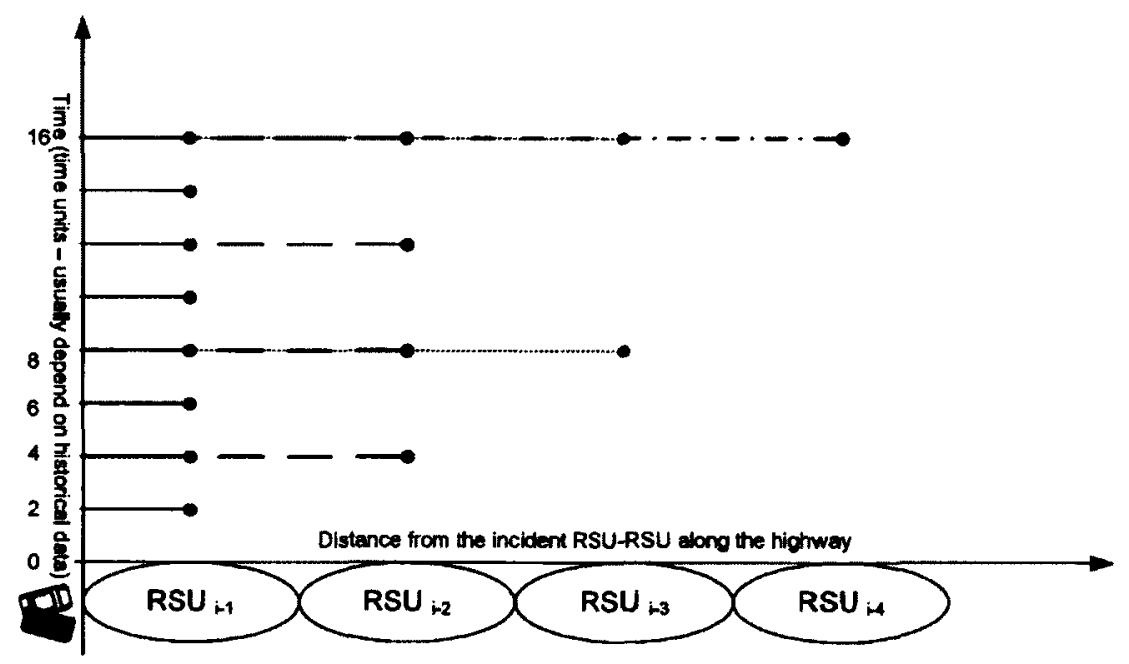

FIG. 51. Illustrating Stage I of information dissemination.

As shown in Figure $51, R S U_{x}$ is notified every $2^{x}$ time units, where $x$ is the RSU number and the value depends on the density of the highway. Stage I in FRIEND protects the highway from flooding of messages [170]. On the other hand, we inform RSU(s) (which notify vehicles) with the incident.

In Stage II, we obey two rules. The first rule is to track the source of the incident to be able to track the movement of vehicles after the event is cleared. The second rule is to send a long time to live message every $T$ seconds, this message targets far away vehicles in order to help drivers to make the decision of keep going or taking an exit. The decision of switching between Stages I and II depends on the average headway distance (AHD), speed of vehicles, and historical data, and time and day of the incident.

\subsubsection{TRACKING THE HEAD AND TAIL OF AN INCIDENT IN STAGE II}

One of the key tasks of Stage II is to keep track of the locations of the Head and Tail of an incident. In this subsection we describe in detail how FRIEND keeps track of the Head and Tail of the incident, as shown in Figure 52. The idea of identifying the area of backup is important as a driver in a traffic backup would like to be updated, but a driver 
coming to a backup would value the information more as the driver has the option to exit before reaching the backup area. So, knowing the length of the backup and tracking the Head and Tail are important information that can be propagated and used in Stage II to inform approaching vehicles of an incident at a specific location. Also it helps safety to know when approaching end of a backup queue.

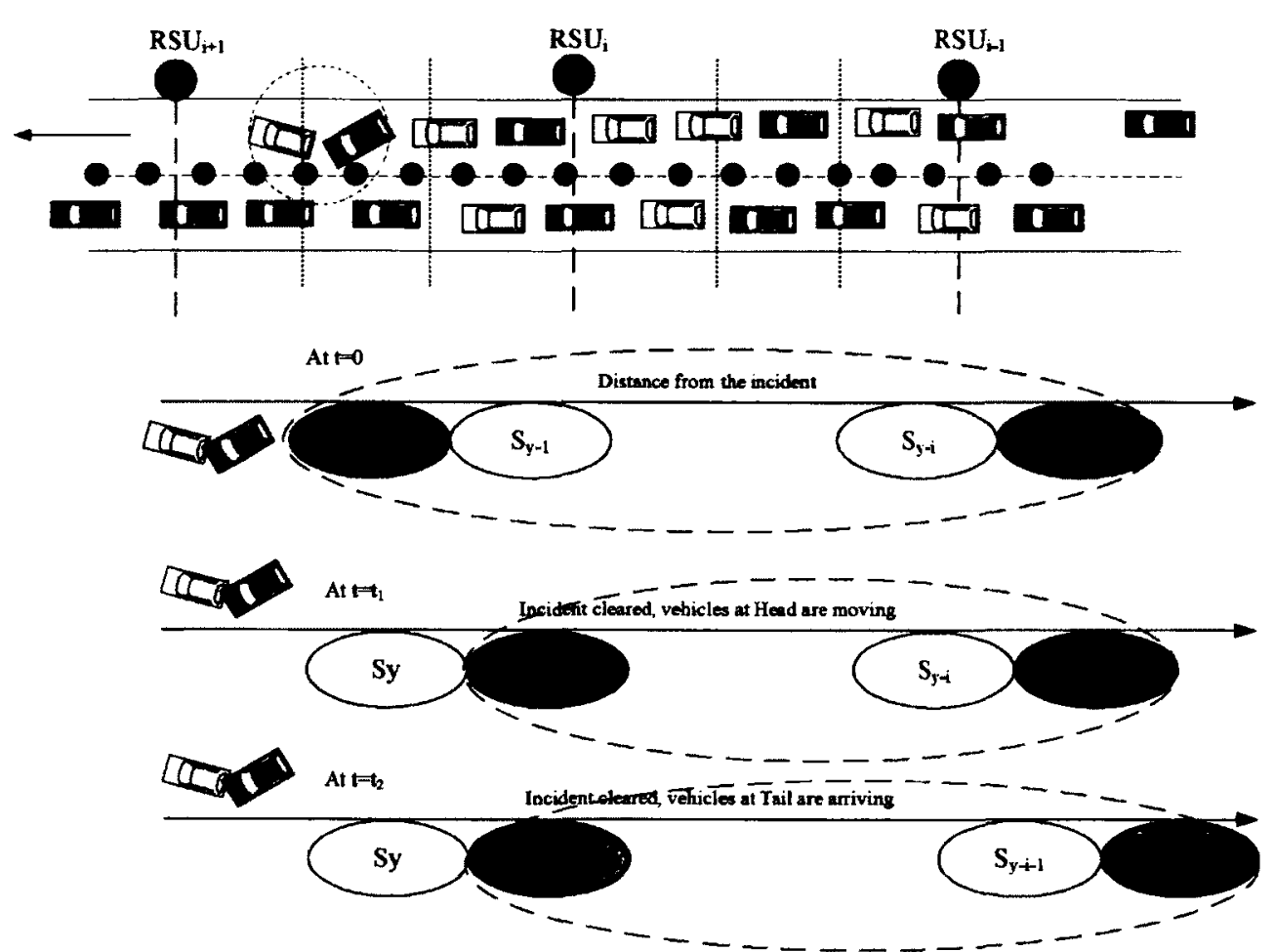

FIG. 52. Illustrating Stage II of information dissemination in FRIEND. At $t=0$, incident just occurred, Head and Tail is identified. At $t=t_{1}$, vehicles started to move and Head is updated. At $t=t_{2}$, vehicles started to arrive in the Tail and Tail is updated.

The following information is sent between adjacent RSU(s):

- Time: the time of last update;

- Head location: The location of the Head of the incident;

- Tail location: The location of the Tail of traffic backup; 
- Incident clearance flag: A bit that shows whether or not the incident has been cleared;

- Average speed of arriving vehicles at the $R S U_{t}$ : where $R S U_{t}$ is the first RSU after Tail;

- Average speed of moving vehicles at the $R S U_{h}$ : where $R S U_{h}$ is the first RSU before Head.

Figure 53 shows the packet information sent between adjacent RSU(s).

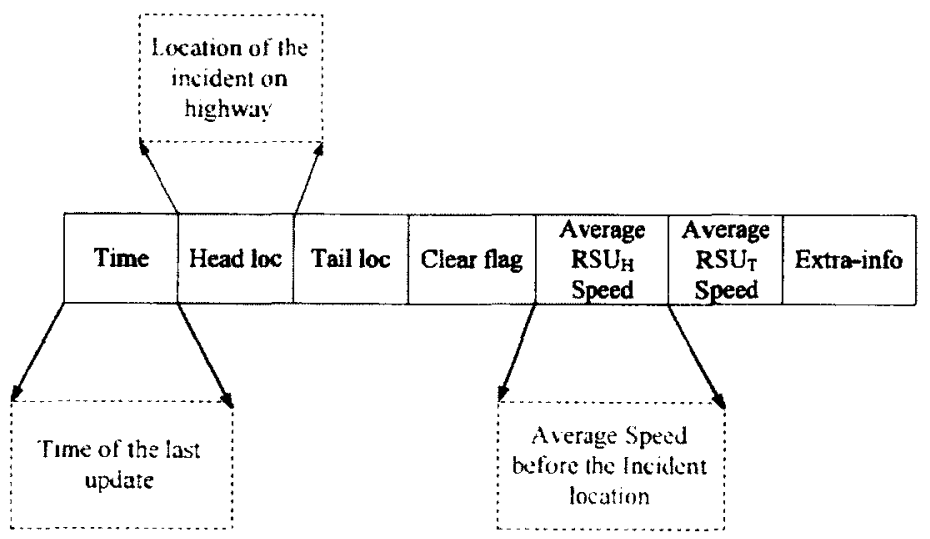

FIG. 53. Illustrating the data exchanged in Stage II of information dissemination.

\subsubsection{THE EXPECTED NUMBER OF VEHICLES THAT PASS BEFORE TURN- ING TO POWER SAVE MODE AT NIGHT}

To save power, we switch all RSU nodes to power save mode at night. Now, let us assume that vehicles pass a certain RSU location according to a Poisson process with parameter $\lambda$. Before allowing the whole cluster to sleep, we wait until no vehicles will come by the next $T$ time units. Then, the expected time RSU will wait before going to power saving mode (sleep) is given by:

Let $X_{1}, X_{2}, \cdots, X_{n}, \cdots$ be the car inter-arrival times, assumed to be independent identically distributed. Let further, $W$ be the random variable that counts the cars that will pass before the cluster can go to power save mode: 


$$
\begin{aligned}
& \operatorname{Pr}[W=k]=\quad \operatorname{Pr}\left[X_{1} \leq T \cap \ldots X_{k} \leq T \cap X_{k+1}>T\right] \\
& =\operatorname{Pr}\left[X_{1} \leq T\right] . \operatorname{Pr}\left[X_{2} \leq T\right] \ldots \operatorname{Pr}\left[X_{k} \leq T\right] . \operatorname{Pr}\left[X_{k+1}>T\right] \\
& =\quad\left(1-e^{-\lambda T}\right)^{k} \cdot e^{-\lambda T}
\end{aligned}
$$

Thus, the expected number of vehicles that pass before turning to power save mode:

$$
\begin{array}{rlc}
E[W] & = & \sum_{k \geq 0} k \cdot\left(1-e^{-\lambda T}\right)^{k} \cdot e^{-\lambda T} \\
& = & \left(1-e^{-\lambda T}\right) \cdot e^{-\lambda T} \cdot \sum_{k \geq 0} k \cdot\left(1-e^{-\lambda T}\right)^{k-1} \\
& = & \left(1-e^{-\lambda T}\right) \cdot e^{-\lambda T} \cdot e^{2 \lambda T} \\
& = & e^{\lambda T-1}
\end{array}
$$

\subsection{SUMMARY}

In this chapter, we explained how making traffic-related decision is done in FRIEND. We started with a reasoning about the sample mean of headway distance. Then, we defined our color states and its mapping from the concept of Level of Service. A Markov chain definition and transitions are described after.

Incident classification is explained in detail. Then, our incident detection algorithm is described. Moreover, we explained the information dissemination mechanism in FRIEND. The proposed technique is divided into two stages. Stage I aims to prevent secondary accidents. Stage II is to track the Head and Tail of an incident. Finally, we calculated the expected number of vehicles to pass before switching to power save mode at night. 


\section{CHAPTER 7}

\section{SIMULATION, EVALUATION AND APPLICATIONS OF FRIEND}

Having presented the theoretical underpinnings of FRIEND in the previous chapters of this thesis, it is now time to turn to an empirical evaluation of FRIEND using simulation. For this purpose, we have settled on the Opportunistic Network Environment (a.k.a. "the ONE simulator") [171]. ONE can generate node movement using different mobility models, and can route messages between nodes using various routing algorithms and sender and receiver types. It allows the user to visualize both mobility and message passing in real time in its graphical user interface. FRIEND testing scenarios differ depending on the different maps, movement of vehicles, messages, routing and reports output as shown in Figure 54. The goal of this evaluation is to build a simulator that can be reflect FRIEND affect on highways.

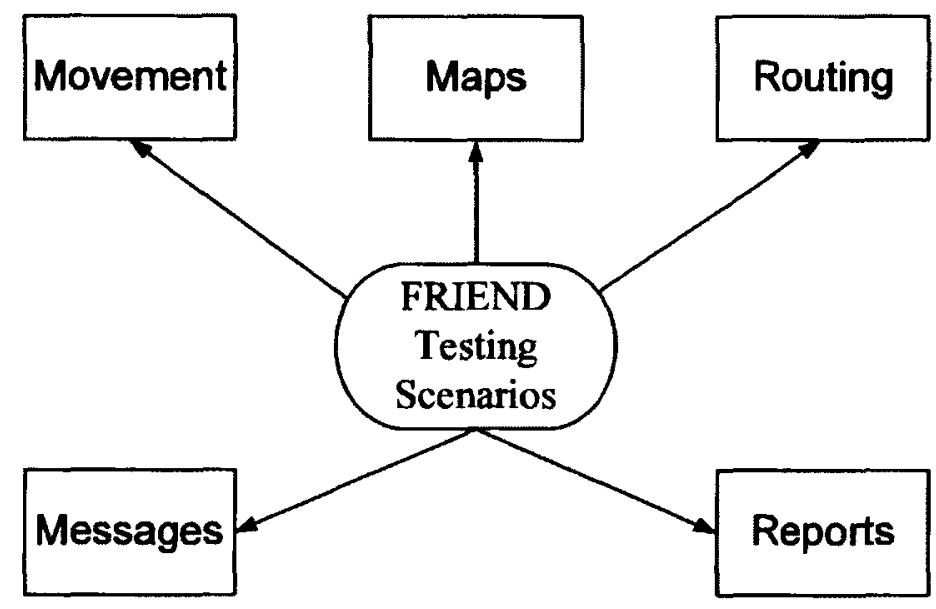

FIG. 54. Block diagram of FRIEND simulator parameters.

\subsection{SIMULATION SETUP AND PARAMETERS}

For the purpose of simulating FRIEND, we adopted a two-lane highway similar to an 11 mile stretch of $U S 13$ highway in Virginia, USA. We generated vehicles randomly from 
the start points. Vehicles can upload the color system in FRIEND for the coming five miles as shown in Figure 55.

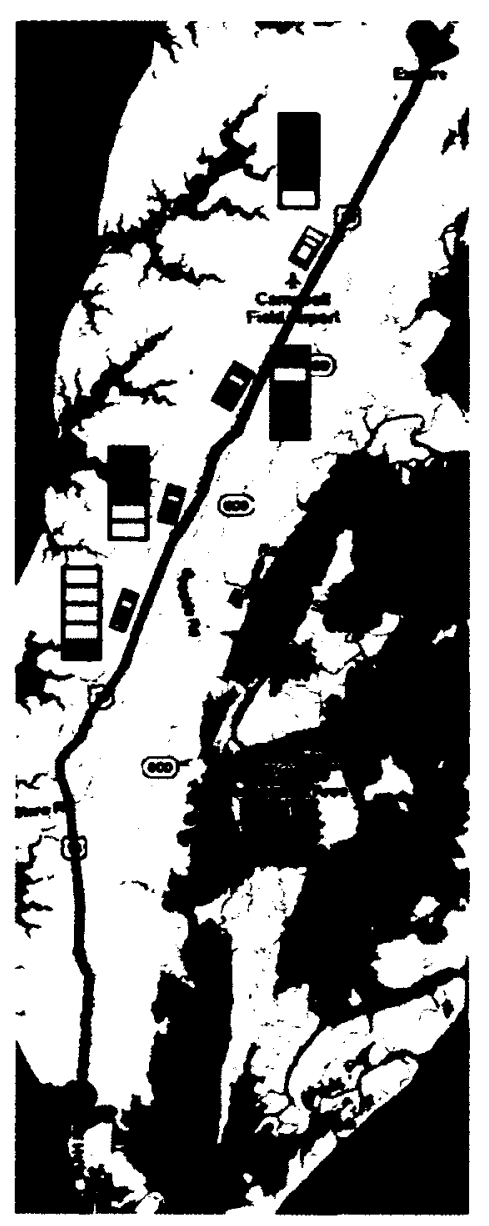

FIG. 55. Illustrating vehicles color-mapped US13 highway in FRIEND.

The model has two types of nodes: fixed nodes and moving nodes. The model assumes fixed nodes between the two lanes, which represent SCEs along the highway. We call these Group I fixed nodes. These stations are 24.384 meters ( 80 feet)[172] apart from each other. We also have another type of fixed nodes every one mile, Group II fixed nodes, which are nodes that communicate with vehicles to upload and download information. Each vehicle, we call them Group III moving nodes, broadcasts a packet every 2 seconds in the range of a circle with radius 12.192 meters ( 40 feet). 


\subsubsection{ASSUMPTIONS}

In our simulation model we make a number of assumptions that represent a simplified (relaxed) version of FRIEND. These simplifying assumptions are discussed next in relation to their impact on FRIEND:

- We assume that the headway distance buffer has size 100 . In other words, we do not have a problem of collecting all information about vehicles and keep it saved through our simulation. The buffer size in the SCEs nodes is of size 5. The buffer size of vehicles is 10 ;

- We assume that we can calculate the density of the RSU-RSU in our simulation. So, we depend on the number of vehicles rather than the headway distance in our simulation. However, in real world scenario, headway distance would be easier to measure if a sufficient number of vehicles are calculating their headway distance as shown before;

- We assume that all nodes are communicate using the same radio technology. This assumption increases the number of packets dropped due to collision between nodes;

- We assume that SCEs are collecting information that can be analyzed later. The SCEs can store infinite amounts of data;

- Finally, historical data is assumed to be given. However, in future work, we would like to study the idea of building our historical database in FRIEND by allowing our system to run for certain amount of time.

\subsubsection{SIMULATION PARAMETERS}

Our simulation uses a two-lane highway of approximately 11 miles. We have three different types of nodes, each with its own buffer that can store a number of records received from other nodes. Fixed nodes have zero speed, while vehicular speed can vary from 0 to $55 \mathrm{miles} / \mathrm{hr}$ which is the maximum speed on $U S 13$. Vehicles use a map-based mobility model. The simulation parameters and values are listed in Table 8. 
TABLE 8. Our simulation parameters.

\begin{tabular}{|c||c|}
\hline Parameters & Values \\
\hline Number of lanes & Two \\
\hline Highway length & $\approx 11$ miles \\
\hline No. of groups on the highway & Three \\
\hline Buffer size for SCEs group & 5 \\
\hline Buffer size for RSU group & 100 \\
\hline Buffer size for vehicle group & 10 \\
\hline Group I and II max speed & zero mile/hr \\
\hline Model movement group I and II & Stationary movement \\
\hline Group III max speed & $100 \mathrm{~km} / \mathrm{hr} \approx 55 \mathrm{mile} / \mathrm{hr}$ \\
\hline Model movement group III & Map based movement \\
\hline Simulation time & $30 \mathrm{~min}=1800 \mathrm{sec}$ \\
\hline GUI map & Head northeast on US-13 $11.2 \mathrm{mi}$ \\
\hline
\end{tabular}

We use OpenJUMP to build our maps, which is an open source Geographic Information System (GIS) written in the Java programming language [173]. OpenJUMP allows you to design your own roads and export it as a wkt file which can be read by ONE simulator.

To simulate mobility, we use a simple map-based mobility model wherein a vehicle starts from the start point and ends at the end of the highway map. In highways, it is easier to get a realistic mobility movement than in cities [174]. However, the ONE simulator offers other various mobility models such as random walks, working day movement, shortest path map based movement, office activities movement, among many others. In the real world, some vehicles enter and exit the highway which can be done in future work to study the effect of these types of events.

To evaluate the performance of FRIEND (modulo the simplifications just mentioned), we ran our simulator to compare the number of

- packets dropped due to collision vs the total number of packets offered;

- consecutive SCEs required as a function of a certain percentage of vehicles detection.

\subsubsection{EVALUATION}


In evaluation, we ran our simulator to produce the following reports:

1. Number of packets dropped due to collision vs the total number of packets with different traffic densities as shown in Table 9. Figure 56 shows the percentage of messages dropped as a function of traffic density.

2. The number of SCEs required consecutively with the percentage of vehicles detection. In this part of the simulation, we study the SCEs without having any RSUs assisting. Our model compares the ratio of messages dropped over all messages that passed in a cluster (we assume that a group of SCEs can be called "cluster") with different cluster sizes. We assume that the cluster size can take values of 2, 3 and 4. We cannot have a cluster of more than four nodes as this will not allow cluster to cluster communication as the range of transmission of SCEs nodes is assumed to be less than 300 feet.

TABLE 9. Total number of messages sent with percentage of collision.

\begin{tabular}{|l||l|l||l|}
\hline Density level & $\begin{array}{l}\text { Total messages } \\
\text { sent }\end{array}$ & $\begin{array}{l}\text { Number of } \\
\text { collisions }\end{array}$ & Percentage \\
\hline Low density & 198,823 & 2,253 & $1.13 \%$ \\
\hline Medium to low density & 241,401 & 3,151 & $1.31 \%$ \\
\hline Medium to high & 288,117 & 5,487 & $1.90 \%$ \\
\hline High density & 397,640 & 12,422 & $3.12 \%$ \\
\hline
\end{tabular}

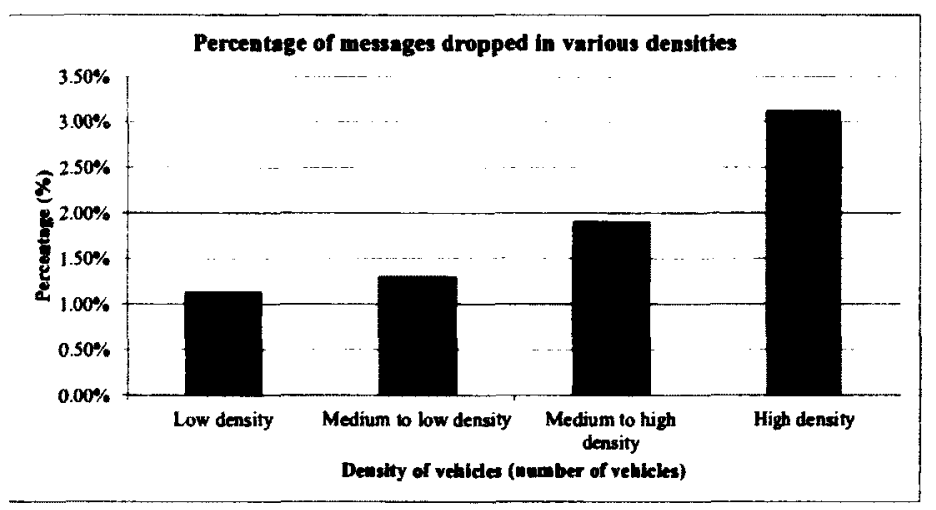

FIG. 56. Illustrating the percentage of message dropped as a function of traffic density. 


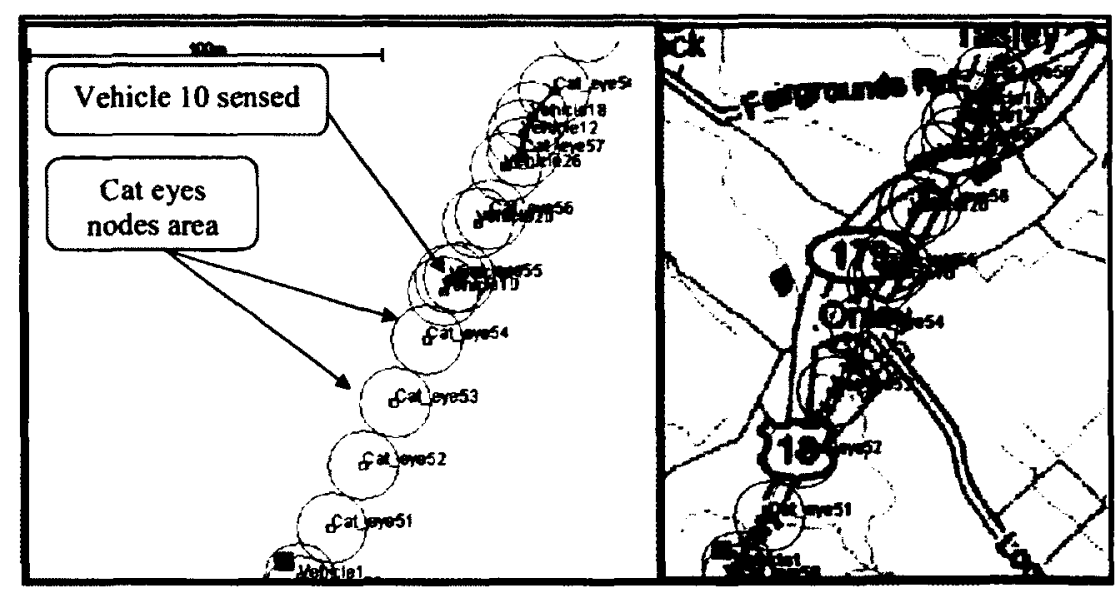

FIG. 57. Simulation- Left A - Right B

Figure 57 shows our simulation; the left side shows our SCEs nodes with the sensing area in the range of 167 feet (50 meters), and the right side shows our map US13.

To evaluate the performance, simulation data is analyzed to get the optimal value of cluster size. In our first scenario, we calculate the cluster size (number of nodes required) in order to detect all vehicles moving with maximum speed of $55 \mathrm{miles} / \mathrm{hr}$. Simulation results are taken and analyzed assuming the three different cases (size of 2, 3 and 4). We expect that the larger the size of the cluster, the more able to detect the vehicles on the highway. At the same time, we cannot increase the cluster size more than four nodes as it will disconnect clusters and prevent cluster communication.

\section{Scenario A: No sleeping mode}

In this case, we study the idea of having two, three, or four SCEs detecting vehicles at high speed. As shown in Figure 58, a four SCE cluster is better at detecting all vehicles moving with maximum speed of $55 \mathrm{mile} / \mathrm{hr}$, while 2 and 3 node clusters suffer from loss.

\section{Scenario B: With sleeping mode}

In the second scenario, we assume that the traffic is low density traffic, which represents the night mode highway traffic or construction on the highway. Our nodes will sleep for 5 mins and wake up for 5 mins. As shown in Figure 59, no vehicles are detected the first 5 mins, then the percentage starts to increase, it reaches about $50 \%$ at the end of the $15 \mathrm{mins}$ then starts to decay at the end. It is also clear that the difference between cluster sizes are 
small. Our explanation that the low density highway does not allow any collision when sensing the vehicles but high speed vehicles still may not be sensed.

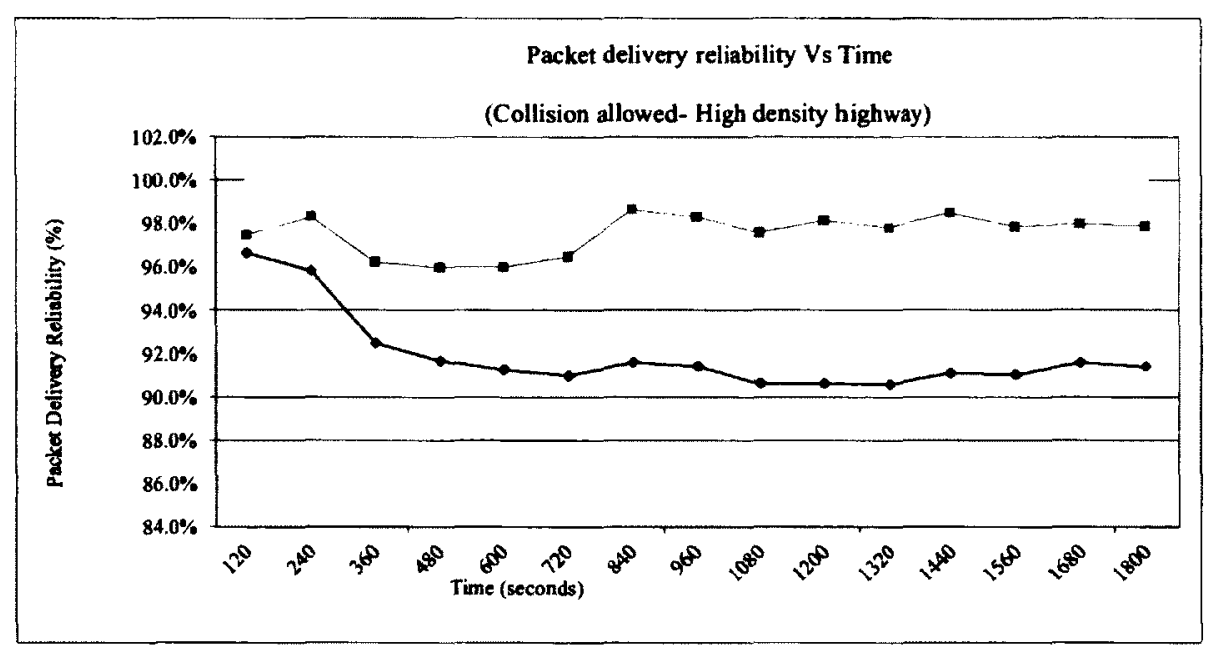

FIG. 58. Simulation- results - no sleeping mode.

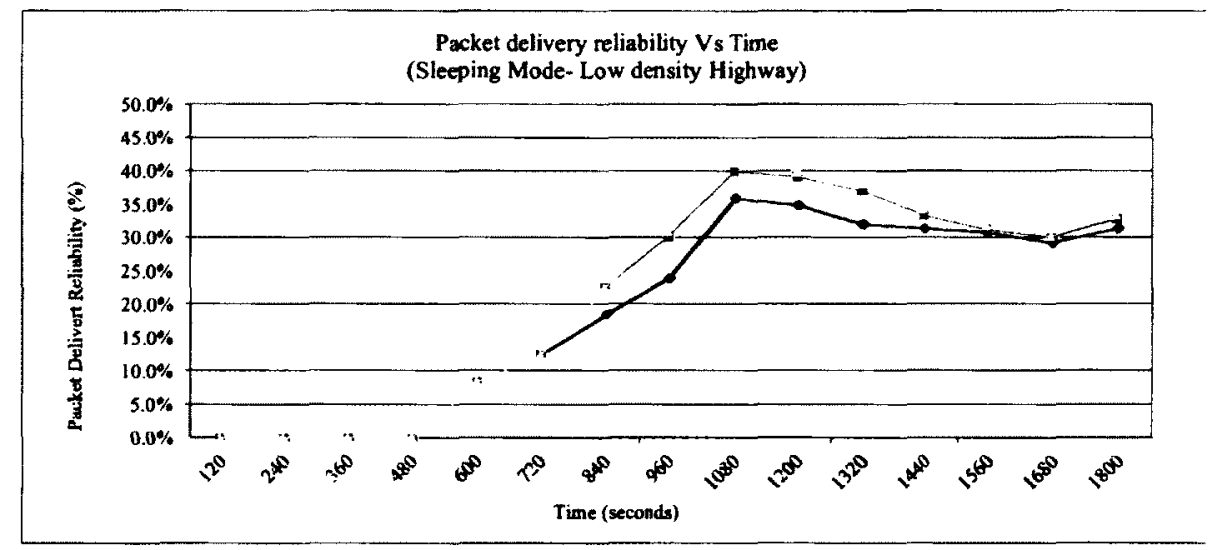

FIG. 59. Simulation- results - sleeping mode

In summary, our results show that four SCEs are sufficient to detect vehicles over the highway and calculate their average speed. Also, our system will be working in case of dead nodes, in case of one or two nodes are dead (or destroyed), SCEs can still calculate 
speed and forward information to other nodes. In case of three consecutive SCEs are destroyed, the cluster will not be able to calculate speed or information but still can forward information. Finally, the case where two consecutive clusters are dead, this will result in a gap in our system which is expected not to happen unless on purpose maintenance.

\subsection{EXAMPLE: A WEATHER CONDITION ALERT SYSTEM}

In Chapter 3, we gave a taxonomy of applications of FRIEND. Our system gives the developers the chance to build different applications over FRIEND. With the amount of data provided and information disseminated, FRIEND can handle many applications from different levels. In this section, we start by introducing the Bayesian network model [175], then we build a simple application over FRIEND. Our idea to show how an application can detect various weather conditions, such as foggy or icy conditions. Each year, approximately 7,000 highway deaths and 800,000 injuries are associated with about 1.2 million weather-related accidents. The estimated annual cost from these weather-related crashes (deaths, injures and property) amounts to nearly $\$ 42$ billion [176]. Alerting drivers of weather conditions including heavy rain, snow, sleet, fog, smoke, dust, ice and black ice can reduce the risks of accidents and improve the safety and efficiency of the highway $[107,110]$.

\subsubsection{THE BAYESIAN NETWORK MODEL}

Bayesian networks are known to be used for updating current beliefs as new information (evidence) becomes available [175]. The basic task of the inference system is to compute the posterior probability upon arrival of some evidences. In our case, new evidences would be - in the case of icy road condition - node temperature values and Electronic Stability Control (ESC) signals. This is called belief updating, or probabilistic inference. We consider the effect of new evidence on the probability of having a weather warning condition. Assume that we know from our data that the probability (our belief) of having a weather warning on a given section of the highway is $p$. For example, in icy conditions, if we noticed that there are some evidences about many ESC signals and low temperatures that are correlated in time and location (we can detect this from EDR data), then we may need to update our beliefs about having a weather warning that may exist and have caused these many correlated ESC signals and low temperature. FRIEND uses a Bayesian network weather warning. We will ignore the case of freezing fog, which is the rare case of both fog and ice happen in the same time. 
Let $\operatorname{Pr}[W W]$ be the probability of weather warning condition. Let $\operatorname{Pr}[F]$ and $\operatorname{Pr}[I]$ be the probability of foggy condition and icy condition respectively. Since, we ignore the case of freezing fog then, we assume that the fog and ice events are independent. Thus, we have

$$
\begin{aligned}
\operatorname{Pr}[W W] & =\operatorname{Pr}[F \cup I] \\
& =\operatorname{Pr}[F]+\operatorname{Pr}[I]-\operatorname{Pr}[F \cap I] \\
& =\operatorname{Pr}[F]+\operatorname{Pr}[I]
\end{aligned}
$$

where $\operatorname{Pr}[F \cap I]=0$

\subsubsection{DETECTING DIFFERENT WEATHER CONDITIONS}

We have two main cases of weather conditions that result in an accident with a high probability, foggy conditions and icy roads. We will discuss the two cases and how to notify drivers with warning messages using our system.

\section{Foggy conditions}

Fog is a visible aggregate of minute water droplets suspended in the atmosphere at or near the surface of the earth. Let $\operatorname{Pr}[F]$ be an priori probability (or belief) of having a foggy condition at a given position on the road. When cameras above cars report a number of low visibility evidences $C$, correlated in both time and location, we update our belief by using Bayesian mechanism. We compute the posteriori probability of a fog condition $F$ at the given location as:

$$
\begin{aligned}
\operatorname{Bel}(F) & =\frac{\operatorname{Pr}[F] \cdot \operatorname{Pr}[C \mid F]}{\operatorname{Pr}[C]} \\
& =\alpha \operatorname{Pr}[F] \cdot \operatorname{Pr}[C \mid F]
\end{aligned}
$$

where $\operatorname{Pr}[C \mid I]$ is the likelihood. $C$ represents any evidence such as camera detection and $\alpha$ is computed by the law of total probability as

$$
\alpha=\frac{1}{\operatorname{Pr}[F] \cdot \operatorname{Pr}[C \mid F]+\operatorname{Pr}[\bar{F}] \cdot \operatorname{Pr}[C \mid \bar{F}]}
$$

Figure 60 shows a vehicle detecting a fog condition using the thermal camera. 


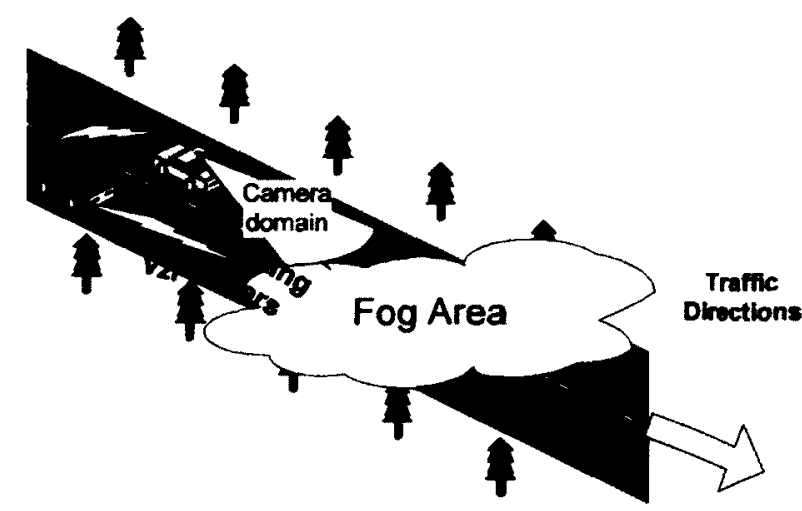

FIG. 60. Vehicle experiencing fog on the highway notifies other vehicles and the infrastructure.

\section{Icy conditions}

In icy conditions, we depend on two factors, the ESC to detect instability situations and temperature measured from the sensors in the nodes over the road. Using the same technique discussed above, we would be able to detect icy conditions and confirm this information using the Bayesian model discussed.

Let $\operatorname{Pr}[I]$ be a priori probability (or belief) an icy condition at a given position on the road. When ESC in the cars report a number of instability conditions evidences $E$, correlated in both time and position, we update our belief by using Bayesian mechanism. We compute the posteriori probability of an icy condition $I$ at the given location as:

$$
\begin{aligned}
\operatorname{Bel}(I) & =\frac{\operatorname{Pr}[I] \cdot \operatorname{Pr}[E \mid I]}{\operatorname{Pr}[E]} \\
& =\beta \operatorname{Pr}[I] \cdot \operatorname{Pr}[E \mid I]
\end{aligned}
$$

where $\operatorname{Pr}[E \mid I]$ is the likelihood, $E$ represents any evidence such as temperature record or ESC signal and $\beta$ is computed by the law of total probability as

$$
\beta=\frac{1}{\operatorname{Pr}[I] \cdot \operatorname{Pr}[E \mid I]+\operatorname{Pr}[\bar{I}] \cdot \operatorname{Pr}[E \mid \bar{I}]}
$$

where $\operatorname{Pr}[E \mid I]$ is the probability of temperature is being recorded low or the probability of the ESC signals given that there is icy condition. Both depend on the sensitivity of the ESC sensors and the sensor placed over the road. 


\subsection{SUMMARY}

In this chapter, we showed how to map our work to a network simulator that can be extended later. We started with the simulation setup and parameters. Then, our assumptions are explained. The evaluation is shown and finally, we gave an example of a simple application (weather application) that used bayesian network model to update our beliefs and decisions. 


\section{CHAPTER 8}

\section{CONCLUDING REMARKS AND DIRECTIONS FOR FUTURE}

\section{WORK}

The main goal of this chapter is to put the work done in this dissertation in perspective: we will present the problems that we have addressed and the solutions we have proposed. We then will point out features of FRIEND that will be implemented as part of future work.

Section 8.1 summarizes the results and the main conclusions of this dissertation. Future extensions and developments of this work are discussed in Section 8.2.

\subsection{CONCLUDING REMARKS}

In this thesis, we proposed FRIEND, a secure and privacy-aware cyber-physical system that automatically detects existing traffic conditions and anticipates discernible trends in the traffic flow, based on which it can intelligently predict imminent traffic events and alert the driving public to their likely occurrence. FRIEND aims to explore the integration of wireless networking with lightweight roadside infrastructure into an embedded system that enables privacy-aware detection and dissemination of traffic-related events.

In summary, the key technical contributions of FRIEND are:

- Laying the theoretical foundations of a scalable, non-intrusive traffic-event detection strategy that is also privacy-aware;

- Exploring the architectural issues and design principles underlying FRIEND;

- Laying the theoretical foundations of detecting traffic-related events based on aggregating collected data; this will allow us to tailor the best information dissemination strategy - we will study formal models that distinguish between dissemination of time-critical and non-time-critical traffic-related events. To the best of our knowledge this is the first study of its kind;

- To the best of our knowledge, FRIEND is the first non-intrusive cyber-physical system that will be able to assist the authorities with managing traffic-related emergencies. 


\subsection{DIRECTIONS FOR FUTURE WORK}

In this section, we describe future research directions to extend FRIEND by the addition of more features. In spite of many interesting and useful features already incorporated into FRIEND, which we perceive to a large extent as a proof of concept, a good deal more can and, perhaps, should be done in the future.

As part of our future work, we plan to continue to improve the current implementation, develop new data collection and aggregation algorithms and conduct scalability studies under various traffic scenarios. We summarize possible extensions to FRIEND in the guise of a bulleted list of "action items" that we plan to address in the near future:

1. Enhance the energy efficiency both of data collection and data dissemination;

2. Exploit existing (or anticipated) correlation of traffic data to put RSUs "to sleep" instead of mandating them to continually collect data. We anticipate that this will safe a great deal of power in dense traffic;

3. Perfect an efficient way whereby the vehicles wake up the RSUs in sparse traffic;

4. Better understand the triggers that signal to FRIEND trends in the traffic flow that need immediate action to prevent congestion from building up (rather than mitigating its effects). One possible outcome is that FRIEND may recommend to the competent authorities to dedicate some lanes as HOV dynamically rather than on a static schedule;

5. Enhance the data aggregation engine of FRIEND. In particular, a more sophisticated version of data aggregation seems to be possible by merging ideas from FRIEND with those from Abuelela's PhD Dissertation [168] which turn out to be quite general;

6. It is important to better assess and evaluate the effect of traffic buildup in the case of a serious incident. One idea is to merge two backups on the highway in the case of different incidents occurring at the same time and impacting the traffic flow;

7. Concerning information dissemination, a number of important issues are still open. Clearly, in order to determine how far to disseminate information about an existing incident it is important to know the following: 
- the expected duration $\Delta$ of the incident, in terms of how long it will take the clear the cause of the incident,

- the expected size of the backup accumulated during $\Delta$,

- the expected speed at which the backup will dissipate, given suitably adjusted historical data about the expected traffic parameters,

- a probabilistic model of discounted value of the disseminated information, mainly in terms of the topology of the affected area;

8. Extending the current simulation model for FRIEND by incorporating more realistic assumptions. 


\section{REFERENCES}

[1] Subramaniam, S. Technical report, University Center of Transportation Research, Virginia Polytechnic Institute and State University, Blacksburg, VA, (1991).

[2] Emily Parkany, C. X. Technical report, The New England Transportation Consortium University of Connecticut, February (2005).

[3] Alibaba.com Hong Kong Limited and Licensors.

[4] Yan, G. and Olariu, S. IEEE Transactions on Intelligent Transportation Systems 12(3), 1 (2011).

[5] US Department of Transportation, Research and Innovative Technology Association. (2011). http://www.bts.gov/publications/national_transportation_statistics/ .

[6] NHTSA National Highway Traffic Safety Administration. http://www-nrd.nhtsa. dot.gov/Pubs/81 1255.pdf, March (2010).

[7] Cambridge Systematics, Inc., with the Texas Transportation Institute. http://www. ops.fhwa.dot.gov/congestion_report/, (2005).

[8] Ryus, P., Vandehey, M., Elefteriadou, L., Dowling, R., and Ostrom, B. Highway Capacity Manual, volume 273. Transportation Research Board, April (2011).

[9] Fontaine, M. Traffic monitoring: in Vehicular Networks: From Theory to Practice. In Olariu and Weigle [12], (2009).

[10] Le, L., Festag, A., Baldesari, R., and Zhang, W. Car-2-X Communications in Europe: in Vehicular Networks: From Theory to Practice. In Olariu and Weigle [12], (2009).

[11] Virginia Department of Transportation. www.virginiadot.org/projects/ constsal-smartrd.asp.

[12] Olariu, S. and Weigle, M. C. Vehicular Networks: From Theory to Practice. CRC Press, Boca Raton, (2009).

[13] Wu, X., Michalopoulos, P., and Liu, H. X. Transportation Research Part C 18, 741 (2010). 
[14] US Federal Highway Administration. http://www.itsdocs.fhwa.dot.gov/JPODOCS/ REPT_MIS/13286.pdf, November (2000).

[15] DARPA. http://www.darpa.mil/grandchallenges/index.asp.

[16] May, A. D. Traffic Flow Fundamentals. Prentice Hall, (1990).

[17] de Dios Ortúzar, J. and Willumsen, L. G. Modelling Transport. Wiley, (2002).

[18] Roess, R. P., Prassas, E. S., and McShane, W. R. Traffic Engineering. Pearson Prentice Hall, Third edition, Erewhon, NC, (2004).

[19] Bai, B., Chen, W., Letaief, K. B., and Cao, Z. IEEE Journal on Selected Areas in Communications 29(1), 161 (2011).

[20] Nzounta, J., Rajgure, N., Wang, G., and Borcea, C. IEEE Transactions on Vehicular Technology 58(7), 3609 September (2009).

[21] Saad, W., Han, Z., Hjrungnes, A., Niyato, D., and Hossain, E. IEEE Journal on Selected Areas in Communications 29(1), 48-60 (2011).

[22] ITS Joint Program Office, US Department of Transporation. http://www. vehicle-infrastructure.org/documents/VIV/Architecture/version2005_07_20.pdf, July (2005).

[23] Lin, X., Lu, R., Zhang, C., Zhu, H., Ho, P.-H., and Shen, X. In IEEE Communications Magazine, volume 46, 88, (2008).

[24] Yan, G., Olariu, S., and Weigle, M. C. Computer Communications 31(12), 28832897 (2008).

[25] Yan, G., Olariu, S., and Weigle, M. Wireless Communications IEEE 16(6), 48 December (2009).

[26] Zhou, T., Choudhury, R. R., Ning, P., and Chakrabarty, K. IEEE Journal on Selected Areas in Communications 29(3), 582 (2011).

[27] Haas, J. J., Hu, Y.-C., and Laberteaux, K. P. IEEE Journal on Selected Areas in Communications 29(3), 595 (2011). 
[28] Calandriello, G., Papadimitratos, P., Hubaux, J.-P., and Lioy, A. In VANET '07: Proceedings of the Fourth ACM International Workshop on Vehicular Ad Hoc Networks, 19 (ACM-VANET, New York, NY, USA, 2007).

[29] Jinyuan, S., Chi, Z., Yanchao, Z., and Michael, F. Y. IEEE Transactions on Parallel Distributed System 21, 1227 September (2010).

[30] Xie, H., Kulik, L., and Tanin, E. IEEE Transactions on Intelligent Transportation Systems 11(1), 61 (2010).

[31] SIRIT-Technologies. http://www.itsdocs.fhwa.dot.gov/research_docs/pdf/ 45DSRC-white-paper.pdf, (2005).

[32] Olariu, S., Khalil, I., and Abuelela, M. Int. J. Pervasive Computing and Communications, 7 (2011).

[33] The National Science Foundation: Program Announcements \& Information. (2009).

[34] Japanese Ministry of Land, Infrastructure and Transportation. www.vics.or.jp/ english/, (2006).

[35] Provera, M. Car2Car Communication Consortium, www.car-2-car.org, June (2005). Slides from IPv6 Task Force Italiana.

[36] Reichardt, D., Miglietta, M., Moretti, L., Morsink, P., and Schulz, W. In Proceedings of the IEEE Intelligent Vehicle Symposium (IVS), (2002).

[37] California Partners for Advanced Transit and Highways. www-path.eecs.berkeley. edu.

[38] Saito, M., Tsukamoto, J., Umedu, T., and Higashino, T. In Proceedings of the IEEE International Conference on Advanced Information Networking and Applications. AINA2005, (2005).

[39] Wischhof, L., Ebner, A., and Rohling, H. In Proceedings of the International Workshop on Intelligent Transportation, 49. WIT, (2004).

[40] Morris, R., Jannotti, J., Kaashoek, F., Li, J., and Decouto, D. In Proceedings of the ACM SIGOPS European Workshop, September (2000). 
[41] Zhang, J., Ziliaskopoulos, A., Wen, N., and Berry, R. In Proceedings of the International IEEE Conference on Intelligent Transportation Systems, 473, September (2005).

[42] Aijaz, A., Bochow, B., Dötzer, F., Festag, A., Gerlach, M., Kroh, R., and Leinmülle, T. In Proceedings of the International Workshop on Intelligent Transportation (WIT), March (2006).

[43] Parno, B. and Perrig, A. In Proceedings of HotNets (HotNets-IV, College Park, Maryland, 2005).

[44] Chisalita, I. and Shahmehri, N. In Proceedings of 56th IEEE VTC-Fall, 1002 (VTCFall, Vancouver, Canada, 2002).

[45] Ott, J. and Kutscher, D. In Proceedings of IEEE INFOCOM (INFOCOM, Hong Kong, China, 2004).

[46] Anda, J., LeBrun, J., Ghosal, D., Chuah, C.-N., and Zhang, M. In Proceedings of the IEEE Vehicular Technology Conference - Spring, 2905 (VTC-Spring, Stockholm, Sweden, 2005).

[47] Dikaiakos, M. D., Iqbal, S., Nadeem, T., and Iftode, L. In Proceedings of the ACM Workshop on Vehicular Ad Hoc Networks (VANET) (VANET, Cologne, Germany, 2005).

[48] Luo, J. and Hubaux, J.-P. Technical Report IC/2004/24, School of Computer and Communication Sciences, EPFL, (2004).

[49] Hubaux, J.-P., Capkun, S., and Luo, J. IEEE Security and Privacy Magazine 2(3), 49 (2004).

[50] Choi, J. Y., Jakobsson, M., and Wetzel, S. In Proceedings of the Ist ACM International Workshop on Quality of Service and Security in Wireless and Mobile Networks (Q2SWinet), 79 (Q2SWinet, Montreal, Canada, 2005).

[51] Raya, M. and Hubaux, J.-P. In Proceedings of the ACM Workshop on Security of Ad Hoc and Sensor Networks, 11 (SASN '05, New York, NY, USA, 2005). 
[52] Sampigethaya, K., Huang, L., Li, M., Poovendran, R., Matsuura, K., and Sezaki, K. In Proceedings of the Workshop on Embedded Security in Cars ((ESCAR), Cologne, Germany, 2005).

[53] Papadimitratos, P., Kung, A., Hubaux, J.-P., and Kargl, F. In Workshop on Standards for Privacy in User-Centric Identity Management (, Zurich, Germany, 2006).

[54] Dötzer, F. In Workshop on Privacy Enhancing Technologies (PET, Cavtat, Croatia, 2005).

[55] Raya, M., Papadimitratos, P., and Hubaux, J.-P. Wireless Communications, IEEE 13(5), 8 October (2006).

[56] Raya, M. and Hubaux, J.-P. In Proceedings of the Swiss Transport Research Conference (STRC) (STRC, Ascona, Switzerland, 2005).

[57] ElBatt, T., Goel, S., Holland, G., Krishnan, H., and Parikhan, J. In Proceedings of ACM VANET, 1 (ACM VANET, Los Angeles, CA, USA, 2006).

[58] Misener, J. A., Sengupta, R., and Krishnan, H. In Proceedings of the 12th World Congress on ITS, November (2005).

[59] Sengupta, R., Rezaei, S., Shlavoder, S. E., Cody, D., Dickey, S., and Krishnan, H. In California PATH Technical Report UCB-ITS-PRR-2006-6, May (2006).

[60] Yin, J., ElBatt, T., Yeung, G., Ryu, B., Habermax, S., Krishnan, H., and Talty, T. In Proceedings of the ACM Workshop on Vehicular Ad Hoc Networks (VANET), October (2004).

[61] Ko, Y.-B., Shankarkumar, V., and Vaidya, N. H. In Proceedings of IEEE INFOCOM, March (2000).

[62] Roy, S., Saha, D., Bandyopadhyay, S., Ueda, T., and Tanaka, S. In Proceedings of ACM Mobihoc (Mobihoc, Annapolis, MD, 2003).

[63] Choudhury, R. R., Yang, X., Ramanathan, R., and Vaidya, N. H. In Proceedings of ACM MOBICOM (MOBICOM, Atlanta, GA, 2002).

[64] Roy, S., Saha, D., Bandyopadhyay, S., Ueda, T., and Tanaka, S. In IEEE 59th Vehicular Technology Conference, volume 4 (VTC -Spring, Los Angeles, CA, USA, 2004). 
[65] Persaud, B. and Hall, F. Transportation Research 23A(2), 103 (1989).

[66] Persaud, B., Hall, F., and Hall, L. Transportation Research Record (1287), 167 (1990).

[67] Ahmed, S. and Cook, A. Transportation Research Record (841), 19 (1982).

[68] Levin, M. and Krause, G. Transportation Research Record (722), 49 (1989).

[69] Stephanedes, Y. and Chassiakos, A. Transportation Research 1C(3), 219 (1993).

[70] Ritchie, S. and Cheu, R. Transportation Research 1C(3), 203 (1993).

[71] Michalopoulos, P. Traffic Engineering and Control 34(2), 66 (1993).

[72] University of Virginia Center for Transportation Studies, Virginia Transportation Research Council. http://www.trb.org/NotesDocs/StateofthePractice/Report.pdf, November (2005).

[73] Smith, B. L., Zhang, H., Fontaine, M., and Green, M. Technical report, Center for Transportation Studies at the University of Virginia, June (2003).

[74] Nelson, D. ITS subsystems and technologies in managing traffic, vehicles and systems., chapter 14. Intelligent Transportation Primer (2000).

[75] Labell, L. and May, A. Research Report UCB-ITS-RR-90-18, Institute of Transportation Studies, University of California, Berkeley, CA, August (1990).

[76] Klein, L. A., Mills, M. K., and Gibson, D. R. Traffic Detector Handbook, volume Volume II. US Department of Transportation, 3 edition, October (2006).

[77] Klein, L. and Kelley, M. Research Report FHWA-RD-95-100, FHWA, U.S. Department of Transportation, December (1996).

[78] Klein, L. Technical report, Artech House, Norwood, MA, (2001).

[79] Coutellier, J.-M. In Traffic Technology Intermational: The 2000 International Review of Advanced Traffic Management, Section 7: Detection and Monitoring, 199200. UK \& International Press, (2000). 
[80] Abramson, N. and Chenoweth, A. In Traffic Technology International: The 2000 International Review of Advanced Traffic Management: Section 7: Detection and Monitoring, number 219. UK \& International Press, (2000).

[81] Washburn, S. and Nihan, N. Journal of Transportation Engineering 125(1), 15-20 (1999).

[82] Pucher, M., Schabus, D., Schallauer, P., Lypetskyy, Y., Graf, F., Rainer, H., Stadtschnitzer, M., Sternig, S., Birchbauer, J., Schneider, W., and Schalko, B. In Intelligent Transportation Systems (ITSC), 2010 13th International IEEE Conference on, 837, September (2010).

[83] Sifuentes, E., Casas, O., and Pallas-Areny, R. Sensors Journal, IEEE 11(8), 1669 August (2011).

[84] Li, S., Zhu, K., van Gelder, B. H. W., Nagle, J., and Tuttle, C. Transportation Research Record: Journal of the Transportation Research Board 1804(0361-1981), 17 Janurary (2002).

[85] Skabardonis, A., Chira-Chavala, T., and Rydzewski, D. Technical Report UCB-ITSPRR-98-1, California PATH Research Report University of California. Berkeley, Janurary (1998).

[86] Li, Z.-X., Yang, X.-M., and Li, Z. Journal of Transportation Engineering 132(7), 565-573 July (2006).

[87] Abuelela, M., Olariu, S., and Weigle, M. C. In Proceedings $65^{\text {th }}$ IEEE Vehicular Technology Conference, 3001 (VTC'08 Spring, Singapore, 2008).

[88] Rawat, D., Treeumnuk, D., Popescu, D., Abuelela, M., and Olariu, S. In Proceedings of the International Workshop on Mobile Vehicular Networks (MoVeNet, Atlanta, GA, 2008).

[89] Treeumnuk, D., Popescu, D. C., and Olariu, S. In Proceedings of the IEEE Workshop on Networking Intelligent Vehicles and Infrastructures (NiVi, Honolulu, Hawaii, 2009).

[90] Varaiya, P., Lu, X.-Y., and Horowitz, R. Proposal, http://path.berkeley.edu/ xylu/ TO6327/TO6327_SEMP.pdf, October (2006). 
[91] Briesemeister, L., Schafers, L., and Hommel, G. In Proceedings of the IEEE Intelligent Vehicles Symposium, 522 (IEEE, Dearborn, MI, USA, 2000).

[92] Briesemeister, L. and Hommel, G. In Proceedings of ACM MobiHoc, 45 (MobiHoc, Boston, MA, USA, 2000).

[93] Sun, M.-T., Feng, W.-C., Lai, T.-H., Yamada, K., Okada, H., and Fujimura, K. In Proceedings of the IEEE Vehicular Technology Conference - Fall, 2685 (VTC-Fall, Boston, MA, USA, 2000).

[94] Bachir, A. and Benslimane, A. In Proceedings of the IEEE Vehicular Technology Conference - Spring, 2456 (VTC-Spring, Seoul, Korea, 2003).

[95] Harras, K. A., Almeroth, K. C., and Belding-Royer, E. M. In Proceedings of IFIP Networking, 1180 (IFIP, Waterloo, Canada, 2005).

[96] Shah, R., Roy, S., Jain, S., and Brunette, W. In Proceedings of the IEEE Workshop on Sensor Network Protocols and Applications (SNPA), 30. SNPA, May (2003).

[97] Hull, B., Bychkovsky, V., Zhang, Y., Chen, K., and Goraczko, M. In Proceedings of ACM SenSys (SenSys, Boulder, CO, 2006).

[98] University Transportation Centers Program, U.S. Department of Transportation (MTC Project 2005-04). Technical report, Iowa State University, (2007).

[99] Wisitpongphan, N., Bai, F., Mudalige, P., and Tonguz, O. In (INFOCOM) Proceedings of the 26th IEEE International Conference on Computer Communications, 2291. INFOCOM, May (2007).

[100] Little, T. and Agarwal, A. In Proceedings of the IEEE Intelligent Transportation Systems, 155, September (2005).

[101] Xeros, A., Andreou, M., Pitsillides, A., and Lestas, M. In Processdings of the Third International Workshop on Vehicle-to-Vehicle Communications, June (2007).

[102] Hafeez, K. A., Zhao, L., Liao, Z., and Ma, B. N.-W. In Proceedings of the International Conference on Systems and Networks Communications, 6, September (2009).

[103] Wu, H., Fujimoto, R., and Riley, G. In Proceedings of the IEEE 60th Vehicular Technology Conference, volume 6, 4548 (VTC-Fall, Los Angeles, CA, USA, 2004). 
[104] Nadeem, T., Shankar, P., and Iftode, L. In Proceedings of the third Annual International Conference on Mobile and Ubiquitous Systems: Networking Services, 1, July (2006).

[105] Abuelela, M. and Olariu, S. In Proceedings of the IEEE Vehicular Technology Conference (VTC-Spring, Barcelona, 2009).

[106] Abuelela, M. and Olariu, S. In Proceedings of the 6th International Workshop on Intelligent Transportation (WIT, Hamburg, Germany, 2009).

[107] El-Tawab, S., Abuelela, M., and Yan, G. In Proceedings of the Ist International Workshop on Intelligent Vehicular Networks with IEEE 6th International Conference Mobile Adhoc and Sensor Systems( MASS '09), 627 (InVeNET, Macau, China, 2009).

[108] Boys, J. T. and Green, A. W. In IPENZ Transactions, volume 24, (1997).

[109] Karpinski, M., Senart, A., and Cahill, V. In Proceedings of the 4th Annual IEEE International Conference on Pervasive Computing and Communications Workshops, 306 (PERCOMW '06, Washington, DC, USA, 2006).

[110] El-Tawab, S. and Olariu, S. International Journal of Information Sciences and Computer Engineering 1(2), 1-6 (2010).

[111] Knaian, A. N. Master's thesis, Massachusetts Institute of Technology, June (2000).

[112] Dittrich, T., Menachem, C., Yamin, H., and Adams, L. Technical report, Tadiran Batteries, (2008).

[113] The PC 104 Consortium. http://www.pc104.org.

[114] Del Corso, D., Passerone, C., Reyneri, L., Sansoe, C., Speretta, S., and Tranchero, M. IEEE Transactions on Aerospace and Distributed Systems 47, 1985 Jul. (2011).

[115] Crimmins, D. M., Patty, C. T., Beliard, M. A., Baker, J., Jalbert, J. C., Komerska, R. J., Chappell, S. G., and Blidberg, D. R. In Proceedings of OCEANS (OCEANS, Boston, Massachusetts, 2006).

[116] Boxwell, M. The Solar Electricity Handbook. Code Green Publishing, Warwickshire, UK, (2010). 
[117] Reddy, T. B. and Linden, D. Linden's Handbook of Batteries. McGraw-Hill, New York, NY, fourth edition, (2011).

[118] Sou, S.-I. and Tonguz, O. IEEE Transactions on Vehicular Technology 60(8), 3586 October (2011).

[119] Aslam, B. and Zou, C. In Proceedings of the IEEE Consumer Communications and Networking Conference, 814. CCNC, Janurary (2011).

[120] US Department of Transporation, Research and Innovative Technology Association. http://www.bts.gov/publications/national_transportation_statistics/, (2011).

[121] Arif, S., Olariu, S., Wang, J., Yan, G., Yang, W., and Khalil, I. IEEE Transactions on Parallel and Distributed Systems 99(2) (2012).

[122] Wang, F.-Y. IEEE Transactions on Intelligent Transportation Systems 11(3), 630 638 (2010).

[123] NHTSA National Highway Traffic Safety Administration. Federal Motor Vehicle Safety Standards 215 Part 49, Code of Federal Regulations Part 563, (2006).

[124] US Department of Transporation, National Highway Traffic Safety Administration. www.nhtsa.gov/DOT/NHTSA/Rulemaking/Rules/Associated/\%20Files/ EDRFinalRule_Aug2006.pdf, Aug. (2006). [Docket No. NHTSA-2006-25666], 49 CFR Part 563.

[125] Gabauer, D. J. and Gabler, H. C. http://www.sciencedirect.com/science/article/pii/ S000145750700139X, (2008).

[126] Sapper, D., Cusack, H., and Staes, L. US-DOT, Office of Research, (2009).

[127] Gabler, H. C., Gabauer, D. J., and Newell, H. L. Technical report, National Cooperative Highway Research Program, December (2004).

[128] Kenney, J. Proceedings of the IEEE 99(7), $1162-1182$ july (2011).

[129] Delphi. http://delphi.com/manufacturers/auto/safety/active/ electronically-scanning-radar/.

[130] Edwin Liebermann - Robert Bosch Corp., editor. Safety and Performance Enhancement: the Bosch Electronic Stability Control (ESP), number 05, Oct. (2004). 
[131] Gabauer, D. J. and Gabler, H. C. Accident Analysis and Prevention 40(2), 548 (2008).

[132] Stoleru, R. and Stankovic, J. In Proceedings of First Annual IEEE Communications Society Conference on Sensor and Ad Hoc Communications and Networks, 430 (SECON, Santa Clara, CA, USA, 2004).

[133] Popescu-Zeletin, R., Radusch, I., and Rigani, M. A. Vehicular-2-X Communication: State-of-the-Art and Research in Mobile Vehicular Ad hoc Networks. Springer; $1 \mathrm{st}$ Edition., May (2010).

[134] Morgan, Y. IEEE of Communications Surveys Tutorials 12(4), 504 -518 quarter (2010).

[135] Lee, E., Yoo, Y., Park, C., Kim, M., and Gerla, M. In Proceedings of the sixth ACM international workshop on VehiculAr InterNETworking, 99. VANET '09, (2009).

[136] Lee, E.-K., Yang, S., Oh, S., and Gerla, M. In IEEE 6th International Conference on Mobile Adhoc and Sensor Systems, 621 (MASS '09, China, 2009).

[137] Yamada, I., Shiotsu, S., Itasaki, A., Inano, S. andand Yasaki, K., and Takenaka, M. In Proceedings of the 4th Workshop on UbiComp Privacy, (2005).

[138] Lee, J.-S., Su, Y.-W., and Shen, C.-C. In Proceedings of the $33^{\text {rd }}$ Annual IEEE Conference on Industrial Electronics Society, 46 (IECON'07, Taiwan, 2007).

[139] Tsai, H.-M., Tonguz, O. K., Saraydar, C., Talty, T., Ames, M., and Macdonald, A. IEEE Wireless Communications 14(6), 67-77 Dec. (2007).

[140] Tufail, A., Fraser, M., Hammad, A., Hyung, K. K., and Yoo, S.-W. In Proceedings of the $12^{\text {th }}$ International Conference on Computer Supported Cooperative Work in Design, 553. CSCWD'08, April (2008).

[141] Wheeler, A. IEEE Communications Magazine 45(4), 70 April (2007).

[142] El-Tawab, S. and Gongjun, Y. In Advances in Vehicular Ad-Hoc Networks: Developments and Challenges, Watfa, M. K., editor. IGI Global (2010).

[143] Santa, J., Gómez-Skarmeta, A. F., and Sánchez-Artigas, M. Comput. Commun. 31(12), 2850 July (2008). 
[144] Blum, J. J., Eskandarian, A., and Hoffman, L. J. IEEE Transactions on Intelligent Transportation Systems 5(4), 347 December (2004).

[145] Kerner, B. S. Congested Traffic Flow: Observations and Theory. Transportation Research Board of the National Academies, (1999).

[146] Mathew, T. V. and Rao, K. V. K. Introduction to Transportation Engineering. NPTEL, (2006).

[147] Luttinen, R. T. Statsical Analysis of Vehicle Time headw. PhD thesis, Helsinki University of Technology Lahti Center, May (1996).

[148] Li, P. Y. and Shrivastava, A. Traffic Flow Stability Induced By Constant Time Headway Policy for Adaptive Cruise Control Vehicles, (2002).

[149] Abtahi, S. M., Tamannaei, M., and Haghshenash, H. Transport 26(4), 375 (2011).

[150] Cowan, R. J. Transportation Research 9(6), 371 (1975).

[151] Greenberg, I. Australian Road Research 2(7), 14 (1966).

[152] Chowdhury, D., Ghosh, K., Majumdar, A., Sinha, S., and Stinchcombe, R. B. Physica A: Statistical and Theoretical Physics 246(3), 471 December (1997).

[153] Griffiths, J. D. and Hunt, J. G. Traffic Engineering Control 32(10), 458 (1991).

[154] Krbalek, D. and Seba, P. Journal of Physics A: Mathematical and General 36(2), L7-L11 May (2003).

[155] Chen, X., Li, L., and Zhang, Y. IEEE Transactions on Intelligent Transportation Systems 11(4), 773 (2010).

[156] Greenberg, I. In Aust. Road Res., volume 2, 14, (1966).

[157] Panwai, S. and Dia, H. IEEE Transactions on Intelligent Transportation Systems 6(3), 314 (2005).

[158] Treiber, M. http://www.mtreiber.de/index.html.

[159] Puan, O. C. Malaysian Journal of Civil Engineering (MJCE) 16(2), 15 Jan (2004). 
[160] Rappaport, T. Wireless Communications: Principles and Practice. Prentice Hall PTR, Upper Saddle River, NJ, USA, 2nd edition, (2001).

[161] Visser, A., Yakali, H., van der Wees, A.-J., Oud, M., van der Spek, G., and Hertzberger, L. IEEE Transactions on Intelligent Transportation Systems 3(2), 120 (2002).

[162] Fenton, L. IEEE Transactions on Communications 8(1), 57 (1960).

[163] Zhou, W. and rui Zhang, S. In Proc. of the Eastern Asia Society for Transportation Studies, volume 4, (2003).

[164] Abuelela, M., Olariu, S., and Stojmenovic, I. In Proceedings of the 5th IEEE International Conference on Mobile Ad Hoc and Sensor Systems, 285. MASS 2008, October (2008).

[165] Tijms, H. C. A first course in stochastic models. Wiley, (2003).

[166] Papacostas, C. S. and Prevedouros, P. D. Transportation Engineering and Planning, volume 3rd ed. Upper Saddle River, NJ: Pearson Education, (2001).

[167] Farradyne, P. B. Traffic Incident Management Handbook. Office of Travel Management, Federal Highway Administration, (2000).

[168] Abuelela, M. A Framework for Incident Detection and Notification in Vehicular Ad-Hoc Networks. PhD thesis, Old Dominion University, August (2011).

[169] Suriyapaiboonwattana, K., Pornavalai, C., and Chakraborty, G. In Proceedings of the IEEE International Conference on Fuzzy Systems, 1639 ((FUZZ), Jeju Island, Korea, 2009).

[170] Oh, S., Kang, J., and Gruteser, M. In Proceedings of the Third Annual International Conference on Mobile and Ubiquitous Systems: Networking and Services, 1, July (2006).

[171] TheOne. In http://www.netlab.tkk.filtutkimus/dtn/theone/, (2009).

[172] VDOT Virginia Department of Transportation. (2009).

[173] OpenJUMP. http://www.openjump.org/, May (2012). 
[174] Wu, A., Ma, J., and Zhang, S. In Proceedings of the 44th Annual Simulation Symposium, 57 (ANSS '11Society for Computer Simulation International, San Diego, CA, USA, 2011).

[175] Korb, K. and Nicholson, A. Bayesian Artificial Intelligent. Chapman and Hall/CRC, (2003).

[176] Lombardo, L. Symposium on Weather Information For Surface Transportation [Available online at http://www.ofcm.gov/wist2/presentationstartpage1.htm], December (2000). 


\section{APPENDIX A}

\section{STATISTICS APPENDIX}

\section{A.1 THE LOG-NORMAL DISTRIBUTION}

The log-normal distribution is a continuous probability distribution of a random variable whose logarithm is normally distributed. If $X$ is a random variable with a normal distribution, then $Y=\exp (X)$ has a $\log$-normal distribution; likewise, if $Y$ is log-normally distributed, then $X=\log (Y)$ is normally distributed. This is true regardless of the base of the logarithmic function: if $\log a(Y)$ is normally distributed, then so is $\log b(Y)$, for any two positive numbers $a$ and $b$.

A variable might be modeled as log-normal if it can be thought of as the multiplicative product of many independent random variables each of which is positive. In wireless communication, the attenuation caused by shadowing or slow fading from random objects is often assumed to be log-normally distributed: see log-distance path loss model.

\section{A.2 SAMPLING}

Sampling techniques have been used to calculate the headway distance between two successive vehicles. We now describe the sampling technique used to calculate the headway time which is very similar to the headway distance (multiplying by speed) [147].

In the study of headways, we assume stationary conditions. Headway $\left(t_{i}\right)$ is the time between two vehicles $(i-1$ and $i$ ) as they pass the RSU on the highway. The mean headway $(\bar{t})$ and the traffic volume $(\tilde{\lambda})$ in a sample are related by:

$$
\tilde{\lambda}=\frac{n}{\sum_{i=1}^{n} t_{i}}=\frac{1}{\bar{t}}
$$

where $n$ is the number of observations in the sample. Since, the expected traffic volume is, the expected mean headway at flow rate $(\lambda)$, which is:

$$
E(\bar{t} \mid \lambda)=\frac{1}{\lambda}
$$




\section{A.3 EULER'S GAMMA FUNCTION - A QUICK REFRESHER}

For all $x>0$, the Gamma function $\Gamma:[0, \infty) \rightarrow \mathbb{R}$ is defined as

$$
\Gamma(x)=\int_{0}^{\infty} e^{-t} t^{x-1} \mathrm{~d} t
$$

We take note of the following classic result involving the Gamma function.

Theorem A.3.1 For all $x>1, \Gamma(x)=(x-1) \Gamma(x-1)$

Proof. Follows by simple integration by parts.

Corollary A.3.2 For all positive integer $x, \Gamma(x)=(x-1)$ !

The Gamma function has a large number of properties of which we mention the following:

- $\Gamma\left(\frac{1}{2}\right)=\sqrt{\pi}$

- $\forall n \in \mathbb{N}, \quad \Gamma\left(n+\frac{1}{2}\right)=\frac{1}{2^{\frac{n-1}{2}}} \int_{0}^{\infty} x^{n} e^{-\frac{x^{2}}{2}} \mathrm{~d} x$

- $\forall n \in \mathbb{N}, \quad \Gamma\left(n+\frac{1}{2}\right)=\frac{1 \cdot 3 \cdot 5 \cdot \cdots \cdot(2 n-1)}{2^{n}} \sqrt{\pi}$

\section{A.4 EULER'S BETA FUNCTION - A QUICK REFRESHER}

For all $m, n>0$, Euler's beta function is defined as

$$
\operatorname{Beta}(m, n)=\int_{0}^{1} x^{m-1}(1-x)^{n-1} \mathrm{~d} x
$$

The following classic result, proved by Euler himself, makes the connection between the gamma and Beta function explicit.

Theorem A.4.1 For all $x, n>0$

$$
\operatorname{Beta}(m, n)=\frac{\Gamma(m) \Gamma(n)}{\Gamma(m+n)}
$$


138

Proof. Several proofs are possible. We evaluate $\Gamma(m) \Gamma(n)$

$$
\begin{aligned}
\Gamma(m) \Gamma(n) & =\int_{0}^{\infty} x^{m-1} e^{-x} \mathrm{~d} x \int_{0}^{\infty} y^{n-1} e^{-y} \mathrm{~d} y \\
& \left.=4 \int_{0}^{\infty} \int_{0}^{\infty} u^{2 m-1} v^{2 n-1} e^{-\left(u^{2}+v^{2}\right)} \mathrm{d} u \mathrm{~d} v \quad \text { [after } x=u^{2}, y=v^{2}\right] \\
& =4 \int_{0}^{\infty} \int_{0}^{\frac{\pi}{2}} r^{2 m-1} \cos ^{2 m-1} \theta r^{2 n-1} \sin ^{2 m-1} \theta e^{-r^{2}} r \mathrm{~d} r \mathrm{~d} \theta \\
& {\left[\text { after } u=r \cos \theta ; v=r \sin \theta, r \geq 0 ; \theta \in\left[0, \frac{\pi}{2}\right]\right] } \\
& =4 \int_{0}^{\infty} r^{2 m+2 n-1} e^{-r^{2}} \mathrm{~d} r \int_{0}^{\frac{\pi}{2}} \cos ^{2 m-1} \theta \sin ^{2 m-1} \theta \mathrm{d} \theta \\
& =2 \int_{0}^{\infty} t^{m+n-1} e^{-t} \mathrm{~d} t \int_{0}^{\frac{\pi}{2}} \cos ^{2 m-1} \theta \sin ^{2 m-1} \theta \mathrm{d} \theta\left[\operatorname{after} t=r^{2}\right] \\
& =\Gamma(m+n) \int_{0}^{\frac{\pi}{2}} \cos ^{2 m-1} \theta \sin ^{2 m-1} \theta \mathrm{d} \theta=\Gamma(m+n) \int_{0}^{1} u^{m-1}(1-u)^{n-1} \mathrm{~d} u\left[u=\sin ^{2} \theta\right] \\
& =\Gamma(m+n) \operatorname{Beta}(m, n)
\end{aligned}
$$




\section{VITA}

Samy S. El-Tawab

Department of Computer Science

Old Dominion University

Norfolk, VA 23529

Samy El-Tawab received his Bachelors Degree in Computer Science with honors from Alexandria University in 2002. In 2006, he received the Master Degree in Computer Science from Alexandria University. His Master's thesis was focused on Redundant Traffic Encoding in VoIP Systems. After that he joined the $\mathrm{PhD}$ program in the Computer Science Department in Old Dominion University where he decided to pursue his dissertation under the supervision of Professor Stephan Olariu in Vehicular Ad-hoc Networks.

Samy's main research interests include a wide variety of networks research topics such as Voice of IP, Vehicular Networks, Network Programming, Wireless Communications and Network Security.

In 2009, Samy's paper was awarded a prize for Excellence in Scholarship in "The College of William and Mary's Eighth Annual Graduate Research Symposium". Samy's research work and ideas were well received by the VANET research community, with over 10 publications in various IEEE journals, conferences and workshops at the time of writing this thesis.

Typeset using LTTEX. 